\title{
MULTICOLOR SURFACE PHOTOMETRY OF 17 ELLIPTICALS ${ }^{\mathrm{a})}$
}

\author{
MARIJN FranX ${ }^{\text {b),c) }}$ \\ Sterrewacht Leiden, P. O. Box 9513, 2300 RA Leiden, The Netherlands \\ and \\ Space Telescope Science Institute, ${ }^{\text {d) }}$ Homewood Campus, Baltimore, Maryland 21218 \\ GARTH ILLINGWORTH ${ }^{\text {) }}$ \\ Space Telescope Science Institute, ${ }^{\text {d) }}$ Homewood Campus, Baltimore, Maryland 21218 \\ and \\ Lick Observatory, ${ }^{\text {e) }}$ University of California, Santa Cruz, California 95064 \\ Timothy HeckMaN ${ }^{\text {f) }}$ \\ Astronomy Program, University of Maryland, College Park, Maryland 20742 \\ Received 22 February 1989; revised 10 April 1989
}

\begin{abstract}
Multicolor two-dimensional surface photometry of 17 elliptical galaxies is presented. Radial profiles have been derived for surface brightness, color, ellipticity, position angle, and the residuals from the fitted ellipses described by $\cos (n \phi)$ and $\sin (n \phi)$ terms, where $n=3$ and 4 . The effects of seeing with noncircular PSFs on the observed brightness profiles and on the ellipticities and position angles have been investigated analytically. At radii as large as 5 times the seeing FWHM, seeing can affect the ellipticity at the $10 \%$ level and introduce uncertainty in the position angles of several degrees, particularly for very round ellipticals. The profiles are compared with data from other authors, and the uncertainties estimated. The agreement is encouraging. The rms mean differences are only 0.02 in ellipticity and $2^{\circ}$ in position angle. Even for the roundest galaxies $(\epsilon \sim 0.03)$, the mean ellipticities and position angles can be determined to such accuracy. Larger differences remain for the surface-brightness profiles. Systematic gradients of \pm 0.1 mag per decade in radius still occur between the surfacebrightness profiles from different authors. The main problem in deriving accurate colors is the uncertainty in establishing the background level on the small-format CCD detectors currently used. The average color gradients in $U-R$ and $B-R$ are -0.23 and -0.07 mag per decade in radius. The formal errors in the color gradients are comparable to the gradients for many galaxies, especially for $B-R$. The color gradients of all galaxies are equal to the mean within the errors. The color gradients are consistent with being due to gradients in metallicity. The observed color gradients would result from a decrease in the metallicity by a factor of $\approx 2$ per decade in radius. The profiles of ellipticity, position angle, and the residuals to the ellipse fitting generally show considerable structure. Deviations from ellipses at the level of $0.5 \%$ are common. The detection of a faint, inclined disklike distortion at a radius of $70^{\prime \prime}\left(\approx 30 \mathrm{kpc}\right.$ for $\left.H_{0}=50 \mathrm{~km} \mathrm{~s}^{-1} \mathrm{Mpc}^{-1}\right)$ in NGC 1700 exemplifies the usefulness of the highorder terms for detecting low-surface-brightness structures. A phase-amplitude representation of the $3 \phi$ and $4 \phi$ terms has also been found to be useful for identifying faint structures. Several cases have been found of structures with constant position angle (phase) in galaxies whose major-axis position angle varies dramatically.
\end{abstract}

\section{INTRODUCTION}

For more than a decade, it has been known that bright elliptical galaxies are supported by anisotropies in their velocity dispersion, and not by rotation, and that this implies that ellipticals might have a triaxial or prolate shape (for a review, see Davies 1987). Because of the ambiguity in the deprojection of observed surface-brightness distributions, no significant progress has been made on the question of the

\footnotetext{
a) Partly based on observations made at the European Southern Observatory, La Silla, Chile.

b) Present address: Center for Astrophysics, 60 Garden Street, Cambridge, MA 02138.

c) Visiting Astronomer at the Kitt Peak National Observatory and the Cerro Tololo Inter-American Observatory, operated by AURA, Inc., under contract with the National Science Foundation.

d) Operated by AURA, Inc., for the National Aeronautics and Space Administration.

${ }^{\text {e) }}$ Present address.

f) Present address: Space Telescope Science Institute, and Johns Hopkins University, Homewood Campus, Baltimore, MD 21218.
}

intrinsic shapes by photometric studies (see, e.g., Schechter 1987). The position-angle twists observed in ellipticals can be explained as the result of the projection of triaxial figures, but cannot straightforwardly be used to determine the triaxiality of ellipticals (e.g., Schechter 1987). Contopoulos (1956), Binney (1978), and Kondrat'ev and Ozernoi (1979) have emphasized that one of the consequences of a triaxial shape is that the projected velocity field shows rotation along the photometric minor axis for some viewing angles. Binney (1985) has constructed a set of kinematic models, and has shown that observations of minor-axis rotation would not only prove that galaxies are not oblate rotators, but would also give information about the intrinsic distribution of shapes. Furthermore, he showed that one has the highest chance of finding minor-axis rotation for apparently round galaxies.

We have started an observational program to measure the minor-axis rotation of a sample of ellipticals, with the goal of establishing the shape distribution for elliptical galaxies. As a first step, we have carried out surface photometry on this sample to determine accurate ellipticities and position angles. Here we report on the results of this photometric study. 
The kinematic observations are presented by Franx, Illingworth, and Heckman (1989).

Apart from our specific interest in the shapes of these galaxies, an important issue that can be addressed with accurate surface photometry of ellipticals is that of radial color gradients. The aperture photometry of de Vaucouleurs and de Vaucouleurs (1972), Sandage and Visvanathan (1978), and Frogel et al. (1978) indicated color gradients within galaxies. With the advent of linear detectors, especially CCDs, it is now possible to measure intensities accurately, and Boroson et al. (1983, 1987), Davis et al. (1985), Cohen (1986), Jedrzejewski (1987), Bender and Möllenhof (1987), and Peletier et al. (1989) have used their CCD data to demonstrate the existence of systematic color gradients. Thus, to contribute to this long-term effort, we decided to expand our program to include images in several colors $(U, B$, and $R$ ), and to derive color profiles.

The multicolor photometry is also useful for the detection of dust. Work by Sadler and Gerhard (1985), Sparks et al. (1985), Lauer (1985b), and Ebneter, Djorgovski, and Davis (1988) has shown that a significant fraction of ellipticals $(20 \%-40 \%)$ have structure attributable to absorption by dust. This structure has been found from the residuals in model-subtracted images, and from the asymmetries in the color images.

In this paper, we present surface photometry on 17 northern and southern ellipticals. In Sec. II the selection criteria for the sample are given, the observations are summarized, and the reduction process is described. One of the important problems in surface photometry is the extent to which the profiles are affected by the blurring of the image due to seeing. In Sec. III an analytical expression is derived for the effects of the point-spread function (PSF) on the intensity, the ellipticity, and the position-angle profiles for the general case of a flattened galaxy observed with a noncircularly symmetric PSF. The results from the surface photometry are presented in Sec. IV and discussed in Sec. V.

\section{DATA ACQUISITION AND REDUCTION}

\section{a) Sample}

Our aim was to obtain multicolor photometry of a fairly wide sample of elliptical galaxies from which we could draw candidates for our spectroscopic observations. The galaxy classifications were taken from the RC2 (de Vaucouleurs, de Vaucouleurs, and Corwin 1976) and the RSA catalogue (Sandage and Tammann 1981). Our selection criteria emphasized flattening, size on the sky, and absolute magnitude. Round, bright galaxies with absolute magnitudes $M_{B}$ between -20 and -22 were given the highest priority (assuming $H_{0}=50 \mathrm{~km} \mathrm{~s}^{-1} \mathrm{Mpc}^{-1}$, as for the rest of this paper). Those galaxies for which no detailed digital surface photometry was published were observed first in the $R$ band. On later nights we took the supplementary $B$ and $U$ frames, and we expanded the sample with $U, B$, and $R$ data for several galaxies with some published photometry. We allowed a small overlap with the samples of Jedrzejewski (1987) and Peletier et al. (1989) for comparison. The sample is by no means complete. It is biased towards round galaxies of intermediate absolute magnitude.

We present here the surface photometry for those galaxies for which we have spectroscopic data, or which are good candidates for future spectroscopic study. We have also included data on a selection of ellipticals observed by others to allow comparison of the results. The galaxies are listed in Table I, where we give catalog classifications, luminosities, sizes, radio fluxes, and IRAS $100 \mu \mathrm{m}$ fluxes.

\section{b) Observations}

The data were taken with the \#10.9 m telescope at KPNO in November 1984, with the $0.9 \mathrm{~m}$ telescope at CTIO in October 1985, and with the $2.2 \mathrm{~m}$ telescope at ESO in December 1985. All the data were taken with RCA CCDs, with scales varying from $0.351^{\prime \prime}$ to $0.860^{\prime \prime}$ pixel $^{-1}$, giving fields of view of $110^{\prime \prime} \times 180^{\prime \prime}$ to $270^{\prime \prime} \times 440^{\prime \prime}$. We have listed the relevant instrumental parameters in Table II. The CCDs had relatively few blemishes. The charge-transfer efficiency along the serial register was poor in the KPNO CCD, resulting in the objects showing a slight asymmetry along the row direction (weak "tails"). Bright stars resulted in unusual charge leakage along columns on this detector.

At all observatories, we used Mould or "nearly Mould" $B$ and $R$ filters. At KPNO and ESO, we used a Schott glass $U$ filter with $\mathrm{CuSO}_{4}$ blocking, and at CTIO a $U$ filter with a similar bandpass but much lower peak throughput. The $U$ and $B$ passbands are comparable to Johnson $U$ and $B$, and the $R$ passband is comparable to Cousins $R$. Integration times were typically $3-5 \mathrm{~min}$ in $R, 10 \mathrm{~min}$ in $B$, and $20-40$ $\min$ in $U$. Bright galaxies were observed with shorter exposure times to avoid saturation in the center. A log of the observations is given in Table III. Most of the $B$ and $R$ exposures had similar signal-to-noise ratios, $\mathrm{S} / \mathrm{N}$. The $U$ exposures had systematically lower $\mathrm{S} / \mathrm{N}$, particularly those taken at CTIO, where the $U$ filter had low throughput. Dark frames were taken for photometric calibration, as were flatfields using both a white painted circular "spot" on the dome and the twilight sky.

\section{c) Reduction and Generation of Profiles}

The raw data were first bias subtracted and divided by the flatfield frames. Since most of the galaxies cover a large part of the CCD, special attention was given to low-spatial-frequency variations in the flatfield. The flatfield was found to vary by up to $1 \%$ on large scales during a run. In some cases we used the twilight-sky exposures to determine the lowspatial-frequency sensitivity variations. We estimated the uncertainty in the large-scale response of the CCD to be $\approx 1 \%$ from comparison of flatfields taken throughout the observing run.

The calibrated images still suffered from CCD defects like bad columns, hot pixels, and cosmic-ray events. We did not eliminate these blemishes, but listed their locations. These flagged pixels were ignored in the analysis of the data. We have applied an ellipse-fitting procedure to these data similar to the procedures used by other authors (e.g., Kent 1984; Lauer 1985a; Davis et al. 1985; Jedrzejewski 1987; Peletier et al. 1989). These authors have shown that the two-dimensional surface photometry of most ellipticals can be parametrized by a set of one-dimensional functions: intensity, ellipticity, and position angle as a function of the semimajor-axis length of the ellipse. Most ellipticals are described to better than $1 \%$ by such a parametrization. For some fraction of ellipticals, it is necessary to add the deviations from the bestfitting ellipse, but this is not a major complication. These deviations are commonly expressed as the amplitude of the residual $\sin (3 \phi), \cos (3 \phi), \sin (4 \phi), \cos (4 \phi)$ terms in intensity along the best-fitting ellipse, where $\phi$ is the position an- 
TABLE I. Sample.

\begin{tabular}{|c|c|c|c|c|c|c|c|c|c|c|}
\hline \multirow{2}{*}{$\begin{array}{l}\text { Galaxy } \\
\text { (1) }\end{array}$} & \multicolumn{2}{|c|}{ Classification } & \multirow{2}{*}{$\begin{array}{l}B_{T} \\
(4)\end{array}$} & \multirow{2}{*}{$\begin{array}{l}r_{e} \\
" 1 \\
(5)\end{array}$} & \multirow{2}{*}{$\begin{array}{l}r_{25} \\
" \\
(6)\end{array}$} & \multirow{2}{*}{$\begin{array}{r}v_{g r p} \\
(7)\end{array}$} & \multirow{2}{*}{$\begin{array}{l}M_{B} \\
(8)\end{array}$} & \multirow{2}{*}{$\begin{array}{l}F_{100} \\
\text { mJy } \\
(9)\end{array}$} & \multirow{2}{*}{$\begin{array}{c}\mathrm{F}_{\text {Radio }} \\
\text { mJy } \\
(10)\end{array}$} & \multirow{2}{*}{$\begin{array}{l}\text { Environment } \\
\text { (11) }\end{array}$} \\
\hline & $\begin{array}{l}\mathrm{RC} 2 \\
(2)\end{array}$ & $\begin{array}{c}\text { RSA } \\
(3)\end{array}$ & & & & & & & & \\
\hline NGC 0636 & E3/E1 & E1 & 12.33 & 19 & 58 & 1865 & -20.53 & - & $<5$ & \\
\hline NGC 1199 & E3/E2 & E2 & 12.44 & 29 & 69 & 2331 & -20.90 & - & $<10$ & N1199 group . \\
\hline NGC 1379 & E0 & E0 & 11.66 & 42 & 93 & 1411 & -20.59 & - & $<6$ & Fornax I. \\
\hline NGC 1395 & E2/E3 & E2 & 10.94 & 45 & 122 & 1585 & -21.57 & $390 \pm 61$ & $<15$ & \\
\hline NGC 1399 & E1P & E1 & 10.55 & 42 & 137 & 1411 & -21.70 & $340 \pm 82$ & 342 & Brightest in core of Fornax I \\
\hline NGC 1404 & E1 & E2 & 10.89 & 27 & 43 & 1411 & -21.36 & $290 \pm 56$ & $<18$ & Fornax I. \\
\hline NGC 1407 & $\mathrm{E} 0 / \mathrm{E} 0$ & E0 & 10.57 & 72 & 144 & 1585 & -21.94 & $480 \pm 74$ & 34 & \\
\hline NGC 1439 & E1/E1 & E1 & 12.07 & 41 & 79 & 1585 & -20.44 & - & $<18$ & \\
\hline NGC 1549 & E0 & E2 & 10.58 & 48 & 140 & 1162 & -21.25 & $180 \pm 55$ & $<13$ & Pair with 1553 . Shells. \\
\hline NGC 1700 & E4/E1T & E3 & 12.26 & 14 & 53 & 4082 & -22.30 & $<654$ & $<3$ & Pair with 1699 . \\
\hline NGC 2986 & E2/E1 & E2 & 11.51 & 41 & 93 & 2188 & -21.70 & $<192$ & 33 & SA spiral at $2.7^{\prime}$. \\
\hline NGC 7144 & E0 & E0 & 11.75 & 40 & 89 & 1861 & -21.10 & $330 \pm 107$ & $<7$ & Pair with 7145. \\
\hline NGC 7145 & E0 & E0 & 11.98 & 39 & 79 & 18,61 & -20.87 & - & $<4$ & Shells to NW. \\
\hline IC 1459 & E3 & E4 & 10.88 & 39 & 119 & 1647 & -21.71 & $1180 \pm 103$ & 890 & Pair with I 5255. \\
\hline NGC 7507 & E0 & E0 & 11.15 & 31 & 97 & 1553 & -21.31 & $<459$ & $<10$ & Pair with 7513 at $18^{\prime}$. \\
\hline NGC 7619 & $\mathrm{E} 2 / \mathrm{E} 1$ & E3 & 12.00 & 32 & 72 & 3493 & -22.22 & - & 22 & Second brightest Pegasus I. \\
\hline NGC 7626 & E1P/E2P & E1 & 11.99 & 38 & 74 & 3493 & -22.23 & - & 165 & Brightest Pegasus I. \\
\hline
\end{tabular}

Notes: Columns (2) and (3) list the galaxy classification of the RC2 and the RSA. Column (4) gives the apparent total magnitude $B_{T}$. Columns (5) and (6) list the effective radius $r_{e}$, and $r_{25}$, the radius of the galaxy at a surface brightness of $25 \mathrm{mag} \operatorname{arcsec}^{-2}$, both in arcsec. Column (7) gives the group velocity, and column (8) the absolute magnitude $M_{B}$ calculated from $B_{T}$ and $v_{g r p}$ with $H_{0}=50 \mathrm{~km} \mathrm{~s}^{-1} \mathrm{Mpc}{ }^{-1}$. The values listed in columns (4) to (7) are taken from Burstein et al. (1987) and Davies et al. (1987). The IRAS $100 \mu \mathrm{m}$ fluxes in column (9) are taken from Jura et al. (1987). The radio fluxes in column (10) are taken from Disney and Wall (1977) and Dressel and Condon (1978). They are at frequencies ranging from 2.4 to $5 \mathrm{Ghz}$. The upper limits are the $3 \sigma$ upper limits from Disney and Wall (1977). The remarks on the environment of the galaxies are taken from the RC2, and references given in Appendix B.

gle with respect to the major axis of the ellipse. The amplitudes are divided by the derivative $r d$ Intensity/dr so that their strengths reflect the deviations of the isophotes from ellipses. Peletier et al. (1989) give examples of the isophotal shapes when such deviations are present. They, along with others (e.g., Lauer 1985b; Bender and Möllenhof 1987), have shown that a majority of ellipticals have deviations from ellipses at a level of $\approx 0.5 \%$.

While this parametrization has the advantage that it gives a very good fit, and directly returns the relevant parameters like intensity, ellipticity, and position angle, it does have the disadvantage that it is determined by an iterative process-it is thus somewhat slow. An alternative parametrization is to express the intensity as a series of harmonical terms

$$
I(r, \phi)=\sum_{n=0}^{k} I_{n}(r) \cos \left\{n\left[\phi-\phi_{n}(r)\right]\right\},
$$

after a suitable choice of the center. The advantage of this

TABLE II. Telescopes and instrumentation.

\begin{tabular}{llll}
\hline \hline & KPNO & CTIO & ESO \\
\hline date. & Nov 14-17,1984 & Oct 8-13, 1985 & Dec 17-19, 1985 \\
telescope. & \#1 0.9-m & $0.9-\mathrm{m}$ & $2.2-\mathrm{m} \mathrm{MPI/ESO}$ \\
CCD & RCA\#1 & RCA\#3 & RCA\#5 \\
pixel size $(\mu \mathrm{m})$ & $30 \times 30$ & $30 \times 30$ & $30 \times 30$ \\
format & $320 \times 512$ & $320 \times 512$ & $320 \times 512$ \\
scale("/pixel) & 0.86 & 0.49 & 0.351 \\
readout noise(e $\left(^{-}\right)$ & 75 & 40 & 50 \\
\hline \hline
\end{tabular}


TABLE III. Log of observations.

\begin{tabular}{|c|c|c|c|c|c|c|c|c|c|c|}
\hline Galaxy & $\begin{array}{l}\text { Band } \\
\text { (2) }\end{array}$ & $\begin{array}{l}\text { Telescope \& } \\
\text { Integration } \\
\text { (3) }\end{array}$ & $\begin{array}{l}\text { PSF } \\
\text { FWHM } \\
(4)\end{array}$ & $\begin{array}{c}\delta^{\delta} \\
(>0.1) \\
(5)\end{array}$ & $\begin{array}{l}\text { Sky } \\
\text { mag } \\
(6)\end{array}$ & $\begin{array}{l}\text { Bkgd } \\
\text { error } \\
\quad(7)\end{array}$ & $\begin{array}{l}\mathbf{r}_{\min } \\
\operatorname{mag} \\
(8)\end{array}$ & $\begin{array}{c}\mathbf{r}_{\min } \\
\epsilon \\
(9)\end{array}$ & $\begin{array}{c}\mathrm{r}_{\min } \\
\Theta \\
(10)\end{array}$ & $\begin{array}{r}\mathbf{r}_{\max } \\
(11)\end{array}$ \\
\hline \multirow[t]{3}{*}{ NGC 636} & $R$ & $\mathrm{~K} 60, \mathrm{~K} 180, \mathrm{E} 300, \mathrm{E} 300$ & 1.0 & & 20.09 & 0.01 & 4.0 & 2.8 & 2.4 & 85 \\
\hline & $B$ & $\mathrm{~K} 800, \mathrm{~K} 800, \mathrm{E} 180, \mathrm{E} 600$ & 1.8 & 0.15 & 22.26 & 0.01 & 7.2 & 6.2 & 5.3 & 85 \\
\hline & $U$ & $\mathrm{~K} 1800, \mathrm{E} 2000$ & $2.2 ?$ & & 21.52 & 0.01 & 8.8 & 6.3 & 5.3 & 85 \\
\hline \multirow[t]{2}{*}{ NGC 1199} & $R$ & K300,K300 & 2.5 & & 20.22 & 0.01 & 10.0 & 8.7 & 4.9 & 94 \\
\hline & $B$ & $\mathrm{~B} 800, \mathrm{~B} 800$ & 2.7 & & 22.42 & 0.01 & 11.0 & 9.4 & 5.3 & 94 \\
\hline \multirow[t]{3}{*}{ NGC 1379} & $R$ & $\mathrm{C} 600, \mathrm{C} 600, \mathrm{E} 180$ & 1.0 & & 21.23 & 0.05 & 4.0 & 4.0 & 4.4 & 70 \\
\hline & $B$ & C300,E240,E300 & 1.1 & & 21.54 & 0.05 & 4.4 & 4.4 & 4.8 & 70 \\
\hline & $U$ & E1800 & 1.0 & & 20.45 & 0.05 & 4.0 & 4.0 & 4.4 & 53 \\
\hline \multirow[t]{3}{*}{ NGC 1395} & $R$ & K300,K300,E180 & 1.3 & & 19.96 & 0.02 & 5.2 & 4.4 & 2.6 & 100 \\
\hline & $B$ & K800,K800,E600 & 1.5 & & 22.43 & 0.02 & 6.0 & 5.0 & 3.0 & 100 \\
\hline & $U$ & $\mathrm{~K} 2000$ & 2.8 & & 21.44 & 0.02 & 11.0 & 9.4 & 5.6 & 100 \\
\hline \multirow[t]{3}{*}{ NGC 1399} & $R$ & $\mathrm{C} 200, \mathrm{C} 600, \mathrm{E} 180, \mathrm{E} 180$ & 1.0 & & 20.85 & 0.07 & 4.0 & 2.8 & 2.4 & 70 \\
\hline & $B$ & $\mathrm{C} 500, \mathrm{C} 1000, \mathrm{E} 300, \mathrm{E} 300$ & 1.0 & & 22.70 & 0.07 & 4.0 & 2.8 & 2.4 & 70 \\
\hline & $U$ & E1800 & 1.1 & & 20.29 & 0.07 & 4.0 & 2.8 & 2.4 & 53 \\
\hline \multirow[t]{3}{*}{ NGC 1404} & $R$ & $\mathrm{C} 200, \mathrm{C} 600, \mathrm{E} 180$ & 0.9 & & 20.88 & 0.05 & 3.6 & 3.1 & 1.7 & 70 \\
\hline & $B$ & C500,C500 & 1.4 & 0.15 & 22.85 & 0.05 & 5.6 & 4.9 & 3.4 & 70 \\
\hline & $U$ & E1800 & 1.1 & 0.14 & 21.66 & 0.05 & 4.4 & 3.8 & 2.6 & 53 \\
\hline \multirow[t]{3}{*}{ NGC 1407} & $R$ & $\mathrm{~K} 300, \mathrm{C} 600, \mathrm{E} 300$ & 1.4 & 0.12 & 18.96 & 0.01 & 5.6 & 6.2 & 5.2 & 129 \\
\hline & $B$ & E600,E900 & 1.7 & & 22.01 & 0.05 & 6.8 & 4.8 & 5.8 & 53 \\
\hline & $U$ & E1800 & 1.5 & & 20.90 & 0.05 & 6.0 & 4.2 & 5.1 & 53 \\
\hline \multirow[t]{3}{*}{ NGC 1439} & $R$ & $\mathrm{C} 200, \mathrm{C} 600$ & 1.8 & 0.12 & 20.69 & 0.03 & 7.2 & 5.6 & 4.7 & 49 \\
\hline & $B$ & C500,C500 & 1.6 & 0.10 & 22.16 & 0.03 & 6.4 & 4.5 & 3.8 & 49 \\
\hline & $U$ & C3000 & 2.5 & 0.23 & 21.79 & 0.07 & 10.0 & 11.0 & 9.0 & 49 \\
\hline \multirow[t]{3}{*}{ NGC 1549} & $R$ & $\mathrm{C} 200, \mathrm{C} 600$ & 1.4 & & 20.93 & 0.07 & 5.6 & 4.9 & 2.8 & 70 \\
\hline & $B$ & $\mathrm{C} 200, \mathrm{C} 1000, \mathrm{E} 300$ & 1.4 & & 22.89 & 0.07 & 5.6 & 4.9 & 2.8 & 70 \\
\hline & $U$ & $\mathrm{C} 2000$ & 2.8 & 0.25 & 21.26 & 0.05 & 11.0 & 13.0 & 8.4 & 70 \\
\hline \multirow[t]{3}{*}{ NGC 1700} & $R$ & $\mathrm{~K} 150, \mathrm{~K} 150, \mathrm{~K} 300, \mathrm{E} 600$ & 1.2 & & 20.28 & 0.01 & 4.8 & 5.9 & 1.6 & 86 \\
\hline & $B$ & K400,K600,E600,E900 & 1.5 & & 22.45 & 0.01 & 6.0 & 7.4 & 2.1 & 86 \\
\hline & $U$ & K2000 & 2.6 & 0.19 & 21.45 & 0.01 & 10.0 & 13.0 & 5.0 & 86 \\
\hline \multirow[t]{3}{*}{ NGC 2986} & $R$ & E180,E300 & 1.4 & 0.10 & 20.73 & 0.05 & 5.6 & 4.9 & 2.8 & 53 \\
\hline & $B$ & $\mathrm{E} 300, \mathrm{E} 480$ & 1.3 & & 22.61 & 0.05 & 5.2 & 4.5 & 2.5 & 53 \\
\hline & $U$ & $\mathrm{E} 1800$ & 1.3 & & 21.85 & 0.05 & 5.2 & 4.5 & 2.5 & 53 \\
\hline \multirow[t]{3}{*}{ NGC 7144} & $R$ & $\mathrm{C} 200, \mathrm{C} 600$ & 1.7 & 0.19 & 20.81 & 0.04 & 6.8 & 9.5 & 7.8 & 55 \\
\hline & $B$ & C1000 (bad guiding) & 3.1 & 0.46 & 22.67 & 0.04 & 12.0 & 26.9 & 22.8 & 55 \\
\hline & $U$ & C3000 & 2.0 & & 21.96 & 0.04 & 8.0 & 8.1 & 6.6 & 49 \\
\hline \multirow[t]{3}{*}{ NGC 7145} & $R$ & $\mathrm{C} 200, \mathrm{C} 600$ & 1.5 & 0.10 & 20.84 & 0.04 & 6.0 & 6.1 & 5.1 & 59 \\
\hline & $B$ & $\mathrm{C} 1000$ & 1.7 & & 22.66 & 0.04 & 6.8 & 6.8 & 5.8 & 59 \\
\hline & $U$ & $\mathrm{C} 2000$ & 2.0 & 0.17 & 21.93 & 0.04 & 8.0 & 10.5 & 9.0 & 49 \\
\hline \multirow[t]{2}{*}{ IC 1459} & $R$ & $\mathrm{C} 600, \mathrm{C} 180^{a}, \mathrm{E} 150^{b}$ & 1.4 & 0.12 & 20.90 & 0.06 & 5.6 & 6.3 & 2.1 & 70 \\
\hline & $B$ & $\mathrm{C} 500, \mathrm{C} 180^{a}, \mathrm{C} 300^{b}$ & 1.0 & & 22.67 & 0.06 & 4.0 & 4.5 & 1.5 & 70 \\
\hline \multirow[t]{3}{*}{ NGC 7507} & $R$ & $\mathrm{C} 100, \mathrm{C} 200, \mathrm{C} 600$ & 1.4 & & 20.73 & 0.05 & 8.0 & 8.1 & 6.6 & 59 \\
\hline & $B$ & C500,C500 & 2.0 & & 22.64 & 0.05 & 8.4 & 8.3 & 7.2 & 59 \\
\hline & $U$ & $\mathrm{C} 2000$ & $?^{c}$ & & 21.77 & 0.05 & 8.4 & 8.3 & 7.2 & 59 \\
\hline \multirow[t]{2}{*}{ NGC 7619} & $R$ & $\mathrm{~K} 394, \mathrm{~K} 300, \mathrm{E} 180, \mathrm{E} 180$ & 1.5 & & 20.17 & 0.01 & 6.0 & 6.8 & 2.3 & 129 \\
\hline & $B$ & E180 & 2.2 & 0.15 & 19.09 & 0.02 & 8.8 & $-^{d}$ & $\omega^{d}$ & 45 \\
\hline \multirow[t]{3}{*}{ NGC 7626} & $R$ & $\mathrm{C} 200, \mathrm{C} 600$ & 2.3 & 0.17 & 20.67 & 0.04 & 9.2 & 8.5 & 7.2 & 65 \\
\hline & $B$ & $\mathrm{C} 1500$ & 2.6 & 0.14 & 22.51 & 0.04 & 10.0 & 8.7 & 7.2 & 65 \\
\hline & $U$ & C3000 & 2.8 & 0.13 & 21.58 & 0.04 & 11.0 & 9.0 & 7.8 & 49 \\
\hline
\end{tabular}

${ }^{a}$ Data made available by Mario Mateo.

${ }^{b}$ Data taken by Reynier Peletier taken on the 1.5-m Danish Telescope at ESO.

${ }^{c}$ No star available to measure seeing.

${ }^{d}$ No reliable shape parameters because of very high sky level

Notes: Column (2) gives the passband filter, Column (3) the telescope ( $\mathrm{C}=\mathrm{CTIO}, \mathrm{E}=\mathrm{ESO}, \mathrm{K}=\mathrm{KPNO})$ and integration time in seconds. Column (4) gives the seeing FWHM in arcsec, column (5) the ellipticity $\delta$ of the PSF if it was larger than 0.1. Column (6) gives the surface brightness of the sky in magnitudes arcsec ${ }^{-2}$, derived from our zero point calibration and the background level on the CCD. The fractional error in the background determination is given in column (7). Columns (8) to (10) give the radii in arcsec at which the effects of seeing on surface brightness, ellipticity and position angle are $0.05,0.02$ and $5^{\circ}$ respectively. For round galaxies $(\langle\epsilon\rangle \leq 0.08)$ these values are $0.05,0.01$, and $5^{\circ}$. Column (11) gives the maximum radius out to which the shape of the galaxy was determined accurately. 
parametrization is that it is strictly linear at constant $r$. Hence the terms $I_{n}(r)$ and $\phi_{n}(r)$ can be determined immediately from $I(r, \phi)$, without iterating. A disadvantage is that the ellipticity is not part of the parametrization. However, to first order the ellipticity is related to $I_{2}$ by

$$
\epsilon(r)=\frac{2 I_{2}(r)}{r d I_{0} / d r} .
$$

The harmonical expansion method has the clear advantage that it is very fast to calculate, and that its linearity produces better error estimates. Our preference, however, was to use a parametrization that included the ellipticity explicitly, like that used by the authors above. We settled, therefore, on an approach whereby the harmonical expansion was used as a preprocessor, as described in more detail below. It is clear that both methods are a filter on the $2 D$ images, where the ellipse-fitting procedure is the more restrictive of the two.

The ellipse-fitting program was an adapted version of the GRASP package, originally written by Mike Cawson (see Davis et al. 1985), and later updated by Lindsey Davis, Robert Jedrzejewski, and Reynier Peletier. The most significant change in the version used here is that at each step the formal errors of all the fitting parameters are determined. The noise in the data was determined at each iteration from the residuals of the fit. In addition, we express the parameters as a function of an "intermediate" axis $m$,

$$
m=\sqrt{a b}=a \sqrt{1-\epsilon},
$$

where $a$ and $b$ are the semimajor and semiminor axes of the fitted ellipse. The main advantage that accrues from this choice of variable is that now the errors in the ellipticity do not correlate with the errors in intensity.

Because of the small angular sizes of our images, we wanted to make the fit over the largest possible range in radius $m$. Thus, we allowed the program to fit ellipses with varying centers, ellipticity, and position angle out to the radius at which only $60 \%$ of the ellipse fell within the image. Outside that radius, the center, ellipticity, and position angle of the ellipses were fixed, and the intensity was determined until the ellipse fell completely off the array. This approach had the advantage that we did not need to use other methods to determine the intensity near the edge of the image.

The data-analysis procedure consisted of two steps, the first involving the use of the harmonical parametrization to allow the removal of stars and bad pixels, and the second the full ellipse fitting. First, the positions of bad regions and bright stars on the image were listed. Then the center of the galaxy was determined accurately by a numerical fit, and the harmonical expansion method was applied, ignoring the listed bad pixels. A difference image was generated that clearly showed the residuals from the fit. This image had a mean of zero, with the stars, bad pixels, and the cosmic-ray events superimposed, allowing a simple and straightforward detection algorithm to be used. All pixels deviating by more than 5-10 times the rms noise were also flagged. The harmonical fitting was repeated, and the residuals were inspected again to check that all bad pixels and interfering stars had been found and flagged. This method is more reliable than any method that tries to determine the bad regions from the original image, while it takes much less time than a full solution of the ellipse-fitting program on an image.

In the second step, the ellipse-fitting procedure was applied to the image, using the parameters from the harmonical fit as initial values. The end result was an intensity profile from the center to the edge of the image, with a maximum radius of about 300 pixels, or $100^{\prime \prime}$ for the ESO data, 150 " for the CTIO data, and 260" for the KPNO data. The structural parameters [e.g., the ellipticity, the $\cos (n \phi)$ terms, etc.] were determined from the center to about 150 pixels, corresponding to 50", 75", and 130", respectively. For small galaxies the structural parameters and the centers of the ellipses were only derived out to smaller radii.

We also derived intensity profiles, positions, and structural parameters for the few bright stars on the residual images. We measured the seeing for all of the exposures from these stellar intensity profiles, and determined the relative pixel scales and orientations of the detectors from the positions of the stars using the data from the three telescopes with the three different filters. We found that the scale values listed in Table II were internally consistent at the level of a few parts per thousand, and not dependent on the color to more than that level. We also found that the ESO and CTIO images were not exactly aligned north-south or east-west. From the position of the stars on exposures of NGC 1407 we determined the relative offsets (less than $2^{\circ}$ ), and from a trailed exposure of a star we determined the absolute offset for the ESO data.

\section{d) Combining Profiles}

For several galaxies we had taken multiple exposures in the same passbands, in some cases with different telescopes. These proved to be essential for establishing the uncertainty in our profiles independently of the errors given by the fitting program.

In general, the intensity profiles were combined by averaging the data, with the inverse formal errors as weights. Only the profiles from the image with the best seeing were used in the central 10". The sky level (see below) was established from the profile from the data with the largest field and the faintest sky; for the intermediate profiles, the sky level was adjusted to ensure agreement in the overlap region. The steps followed and the various tests were:

Nonlinearities. Comparison of the intensity profiles showed no saturation effects or nonlinearities in the detectors. Since the weather conditions were not photometric during the observations, we could not use the absolute intensity ratios of the images to compare them with the exposure times as a check on nonlinearity. The comparison could not, therefore, detect all the nonlinear effects in the data. For example, if the measured intensity $I^{\prime}$ is related to the real intensity $I$ by

$$
I^{\prime}=I^{1+\alpha}
$$

where $\alpha$ is some small number, then the ratio of two images with different exposure times is still independent of the intensity, but the ratio itself is not linear with the ratio of the exposure times. Thus these comparisons can only give lower limits to such a nonlinearity. However, these CCDs have been used extensively and no significant nonlinearities have been reported.

Shape comparison. For nine galaxies, we had profiles from different telescopes, allowing us to check for systematic effects. As a check on possible differences between different telescopes, we analyzed these profiles separately. We found that the structural parameters agreed very well; the rms mean position-angle difference was $2^{\circ}$, while the rms mean ellipticity difference was 0.02 .

Background determination. The largest source of uncertainty in the intensity and color profiles results from the 
difficulty in establishing the "sky" level in the data. This was clearly a problem, since all the profiles from the ESO and CTIO telescopes showed a gradient in intensity at the outer radius, i.e., at the edges of the image at a typical radius of $80^{\prime \prime}-100^{\prime \prime}$. It is obvious that we can make significant errors by taking the intensity in the corners of the array as the background. To improve this situation, we decided to fit a power law $I=m^{\alpha}$ plus constant offset (where the radius $m=\sqrt{a b}$ was defined above) to the intensity profiles at radii larger than 50". This choice of radius allowed the fits to be applied to each CCD dataset over some common range in radius. The power law index $\alpha$ was chosen to be -2.5 , and we repeated the fit with an index of -2 and -3 to get an estimate of the error. These indices are typical of elliptical galaxy intensity profiles near $r_{\mathrm{e}}$ (see, e.g., Tonry 1987). Each point had comparable weight in the fit. This approach was used to determine backgrounds for all of the profiles. The fits were visually inspected, and obvious bad points in the profile were deleted.

For the KPNO data, which extended to $180^{\prime \prime}$, the background differed by less than $1 \%$ from the outermost intensity value, and was independent of the power law index of the fitted model. For the ESO and CTIO data, which extended only to $100^{\prime \prime}-120^{\prime \prime}$, the differences between the background and the outermost intensity value were generally large, in the most extreme case 10\%-15\% (for NGC 1399 and NGC 1549 ) and dependent on power law index. An index of -2 would give a background lower by $10 \%$ for these two galaxies. One might try to improve upon this situation by fitting another model, such as an $r^{1 / 4}$ law. We have not attempted to use such a model, because this requires the estimate of an uncertain scale factor, and because the intensity profiles of ellipticals differ significantly enough from each other to prohibit an accurate fit with any one such model. Hence we have used the background as determined above, and we have made a reasonable estimate of the error in this procedure. We have estimated the uncertainty for galaxies like NGC 1399 and NGC 1549 to be 7\%, for the smaller galaxies observed at ESO and CTIO to be $3 \%-5 \%$, and for the galaxies observed at KPNO to be $1 \%-2 \%$. The lower limit is set by the accuracy of the flatfield, which we have estimated at $1 \%$. The uncertainties are listed in Table III in column 7 . We attempted to use aperture photometry in the literature to constrain and improve our "sky" levels, but found that the photometry did not have the high level of accuracy that is required for this approach.

Aperture photometry. Finally, we used published aperture photometry to calibrate the magnitude zero points of our data. We used the compilation of Lauberts and Sadler (1984) for the southern galaxies, supplemented by the photometry of Sandage $(1973,1975)$, Sandage and Visnavathan (1978), Persson, Frogel, and Aaronson (1979), Mould (1981), and Burstein et al. (1987). We used the conversion relations given by Sandage and Visnavathan (1978) to convert their data to the standard Johnson $U B R$ system. Aperture data whose values deviated significantly (by $\approx 0.08-0.1$ mag) from the mean zero-point value, as well as data with apertures larger than 120 ", were not included in the calibration. As a check on the calibration errors caused by the uncertainty in the background, we repeated the calibration with the background as one of the free parameters. In most cases the change in the zero point was negligible, being on the order of a few hundredths of a magnitude. Since the color gradients in ellipticals are small, we did not include any color terms in the calibration. The error in the calibration is dependent upon the errors in the literature data, and on the error in the background. The rms mean difference between the aperture photometry and that derived from our synthetic apertures is only $0.04 \mathrm{mag}$. Given this, it seems reasonable to take the likely error in the zero points to be $\approx 0.05 \mathrm{mag}$.

Internal comparison. As a check on the overall data-reduction procedure, we have derived and calibrated profiles from the CTIO, ESO, and KPNO telescopes separately for NGC 636, NGC 1379, NGC 1395, NGC 1399, NGC 1549, and NGC 1700. We have differenced the profiles for the same passband, and we also give the color profiles, when available for any pair of telescopes. The profiles are presented in Fig. 1. We list in Table IV the mean logarithmic gradient of the differences in the intensity profiles (e.g., $\langle\Delta R /$ $\Delta \log r\rangle$ ) between the telescopes, and the color gradients $(\langle\Delta C / \Delta \log r\rangle)$ for each telescope with galaxies in common. The differences in the same bandpass are generally smaller than the errors predicted by our background uncertainty. Furthermore, the color gradients measured at the different telescopes agree very well, despite the fact that the observations had very different scales. The errors of the color profile were calculated by taking the square root of the sum of the squared errors in each passband. We conclude that the errors in the color gradients are overestimated in this way, most probably because the errors in the background determination are in the same direction for both profiles taken in different passbands. In fact, it is interesting to note the excellent agreement between the reliable colors from the KPNO data, where the frames are large enough to allow the "sky" to be well established, and the ESO data, with its very poorly determined sky levels, as reflected in the large errors. An optimist would conclude from this that our sky estimates are subject to errors smaller than those we indicate. Alternatively, the background uncertainties from the individual ESO frames may be correlated in sense and magnitude so as to allow such close agreement with the KPNO data. Which of these options is more correct must be decided with larger CCDs. The literature comparisons shown later suggest that the former option may be more appropriate.

The last step to be taken is the determination of the interval in radius where effects due to seeing, guiding errors, and background uncertainty are within prescribed limits. The effects of background uncertainty can be derived from the error estimates discussed above, and tabulated in Table III. The effect of seeing on the intensity profiles and on the structural profiles such as ellipticity and position angle are not as straightforward to determine. We deal with this in detail in the next section.

\section{SEEING EFFECTS ON TWO-DIMENSIONAL SURFACE PHOTOMETRY}

Schweizer $(1979,1981)$ was the first to stress the importance of seeing on observed parameters like the core radius and the central surface brightness of galaxies. He showed that these effects can be significant even if the observed core radius is much larger than the seeing, and that they depend not only upon the FWHM of the stellar point-spread function (PSF), but also on the wings of the PSF. Further work (e.g., Bailey and Sparks 1983; Kormendy 1984) confirmed his analysis. Peletier et al. (1989) numerically evaluated the effects of seeing on the observed structure of galaxies, and found large effects on the ellipticity, and noted that the effect 

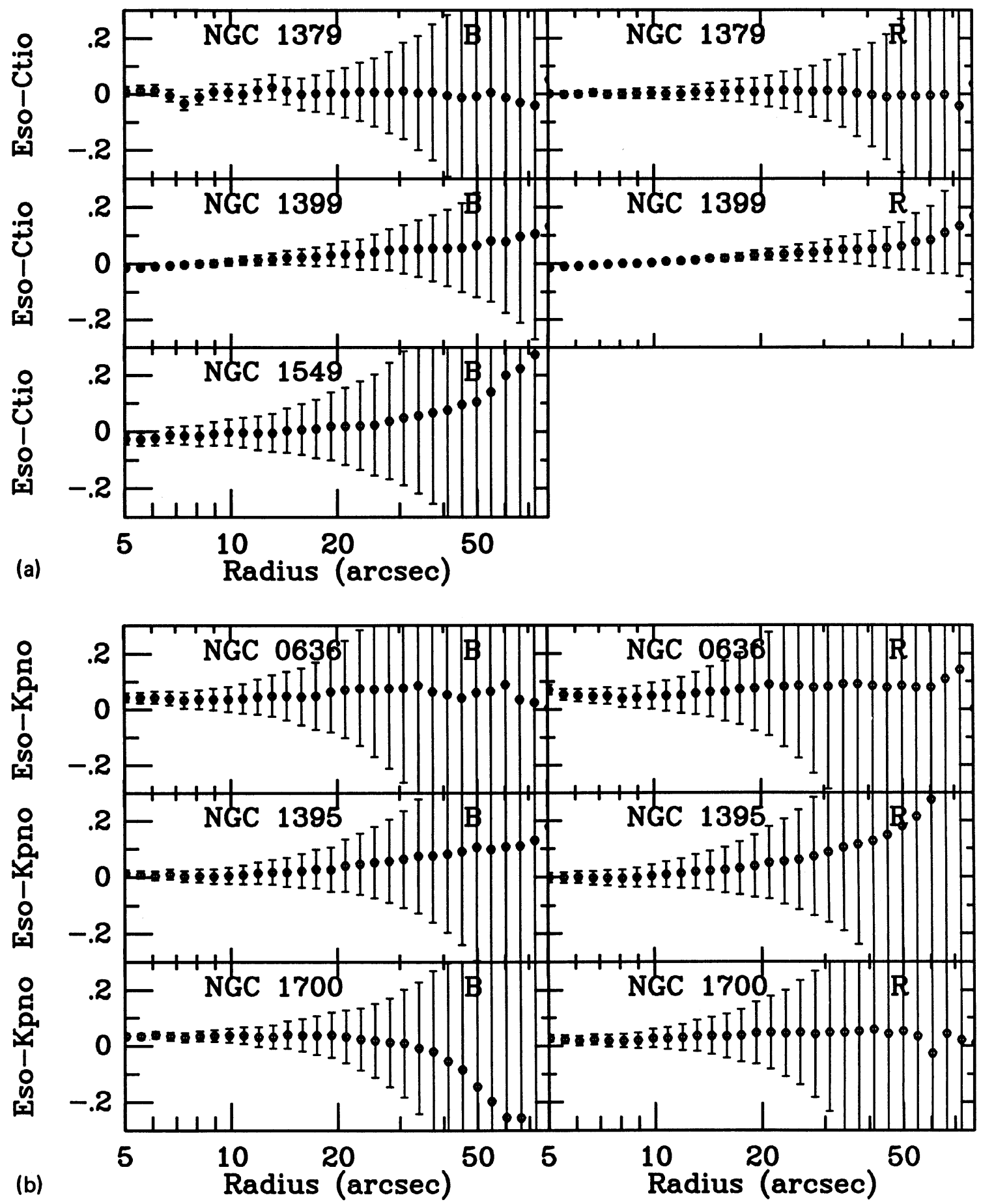

FIG. 1. Differences in magnitude between data from different telescopes. The difference between the ESO and CTIO data is plotted in (a), and between ESO and KPNO in (b). The color gradients of these galaxies from the different telescopes are shown in (c). The very large error bars seen in some cases arise from the (systematic) uncertainty in the background determinations. The correlation between the errors in the background for different passbands on a given telescope leads to an overestimation of the errors in the colors, by an amount that cannot be readily determined. 

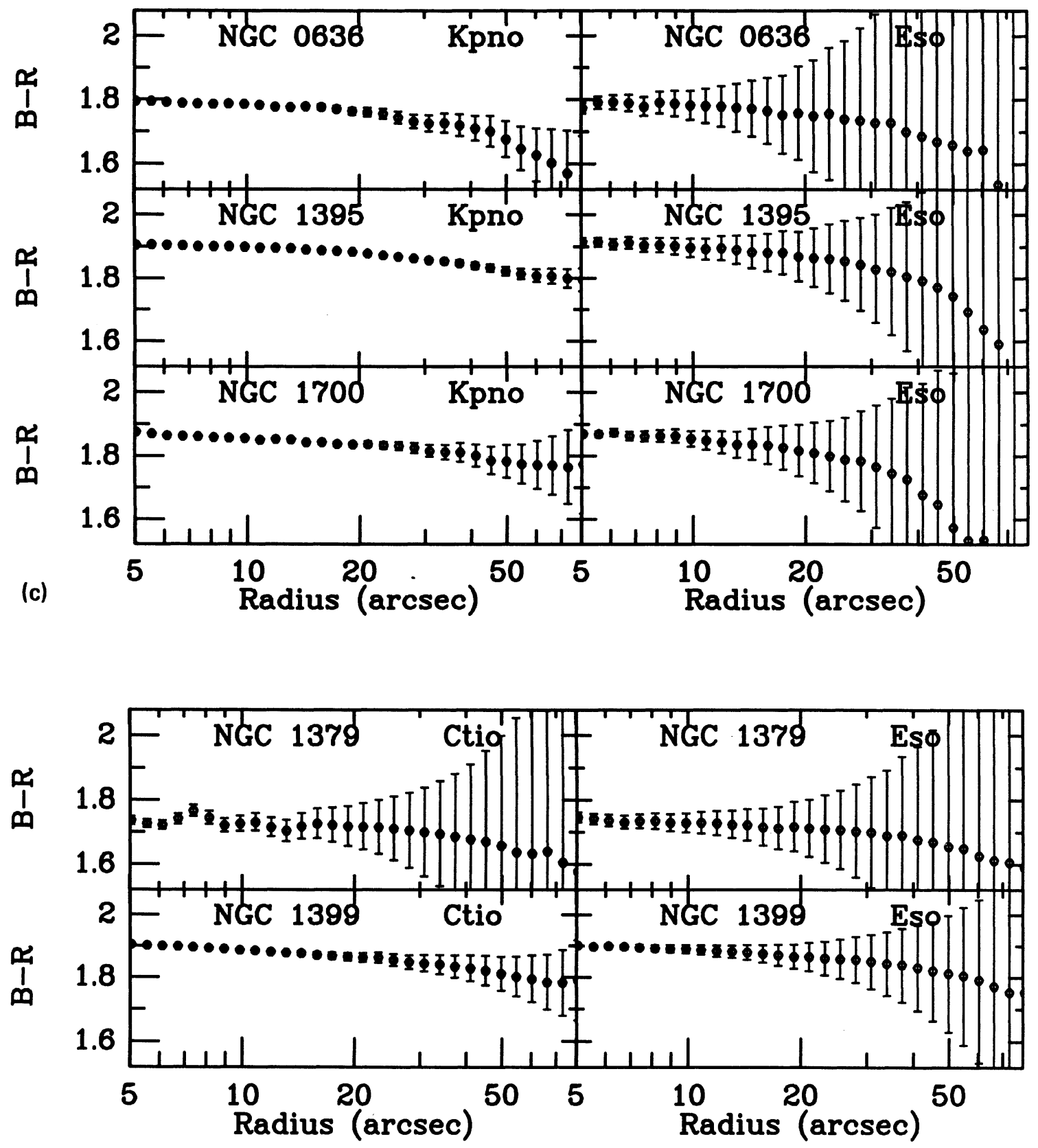

Fig. 1. (continued) 
TABLE IV. Comparison of data from different telescopes.

\begin{tabular}{cccccccc}
\hline \hline Galaxy & \multicolumn{2}{c}{ ESO-KPNO } & \multicolumn{2}{c}{ ESO-CTIO } & ESO & CTIO & KPNO \\
& $\frac{\Delta B}{\Delta \log r}$ & $\frac{\Delta R}{\Delta \log r}$ & $\frac{\Delta B}{\Delta \log r}$ & $\frac{\Delta R}{\Delta \log r}$ & $\frac{\Delta(B-R)}{\Delta \log r}$ & $\frac{\Delta(B-R)}{\Delta \log r}$ & $\frac{\Delta(B-R)}{\Delta \log r}$ \\
$(1)$ & $(2)$ & $(3)$ & $(4)$ & $(5)$ & $(6)$ & $(7)$ & $(8)$ \\
\hline NGC 636 & $0.05 \pm 0.33$ & $0.05 \pm 0.35$ & & & $-0.07 \pm 0.32$ & & $-0.07 \pm 0.02$ \\
NGC 1379 & & & $0.01 \pm 0.15$ & $0.02 \pm 0.10$ & $-0.05 \pm 0.15$ & $-0.04 \pm 0.12$ & \\
NGC 1395 & $0.07 \pm 0.16$ & $0.10 \pm 0.23$ & & & $-0.09 \pm 0.16$ & & $-0.05 \pm 0.01$ \\
NGC 1399 & & & $0.08 \pm 0.08$ & $0.07 \pm 0.04$ & $-0.06 \pm 0.08$ & $-0.07 \pm 0.08$ & \\
NGC 1549 & & & $0.08 \pm 0.23$ & & $-0.12 \pm 0.17$ & & $-0.07 \pm 0.02$ \\
NGC 1700 & $-0.02 \pm 0.18$ & $0.04 \pm 0.25$ & & & -0.07 & \\
\hline \hline
\end{tabular}

Notes: Columns (2) and (3) give the mean logarithmic gradients of the point-to-point differences in $B$ and $R$ for the ESO and KPNO data. The gradients have been determined in the interval from $5^{\prime \prime}$ to $30^{\prime \prime}$. Columns (4) and (5) give the same gradients for the ESO and CTIO data. Columns (6), (7) and (8) give the logarithmic color gradients for the data from each telescope separately.

depends upon the ellipticity of the galaxy. The sample of galaxies presented here contains many round galaxies, and one of our concerns was the degree to which a noncircularly symmetric PSF affected the observed position angles and ellipticities. Guiding errors, focusing errors, bad alignment of the optics, and charge-transfer effects on the CCD can cause noncircular PSFs. In the following, we derive analytical expressions for the effects of a noncircularly symmetric PSF on intensity, ellipticity, and position angle. The goal here is not to determine corrections to the observed profiles for the effects of seeing, but to identify the radii at which the profiles become unreliable at a well-defined level (e.g., $10 \%$ ).

We parametrize a family of PSFs $f$ with similar profiles but different scales $s$. It is assumed that the PSF is constant on similar, concentric ellipses, with

$$
f(x, y)=\frac{1}{s^{2}} g\left(\frac{x^{\prime 2} / p+p y^{\prime 2}}{s^{2}}\right),
$$

with $p \geqslant 1$ and

$$
\left(\begin{array}{l}
x^{\prime} \\
y^{\prime}
\end{array}\right)=\left(\begin{array}{cc}
\cos \psi & \sin \psi \\
-\sin \psi & \cos \psi
\end{array}\right)\left(\begin{array}{l}
x \\
y
\end{array}\right) \text {. }
$$

The parameter $p$ is the elongation of the PSF, and is related to the ellipticity $\delta$ of the PSF by $\delta=1-1 / p$. The angle $\psi$ is the position angle of the longest axis of the PSF, measured anticlockwise from the $x$ axis. The second-order moment $G_{2}$ of $g$ is defined by

$$
G_{2}=\int r^{2} g\left(r^{2}\right) d \mathbf{r}
$$

which is independent of the scale $s$, the elongation $p$, and angle $\psi$. The second-order moment $F_{2}$ of the PSF is

$$
F_{2}=\int r^{2} f(\mathbf{r}) d \mathbf{r}=s^{2} G_{2} \text {. }
$$

We assume that the surface brightness of the galaxy is constant on similar concentric ellipses, and that the $x$ axis and the $y$ axis are symmetry axes. We write

$$
I(x, y)=J\left(m^{2}\right),
$$

with

$$
m^{2}=\left(x^{2} / q\right)+q y^{2} .
$$

Hence the ellipticity of the galaxy is equal to $\epsilon=1-1 / q$. Note that $I$ denotes the intensity of the galaxy at an arbitrary point, while $J$ denotes the one-dimensional profile of the galaxy. In Appendix A we derive the seeing effects on the observed intensity, ellipticity, and position angle to second order in $s$. For small ellipticities $\epsilon$ and $\delta$ of the galaxy and of the PSF, respectively, the errors are

$$
\begin{aligned}
& \Delta J=\left(J^{\prime}+m^{2} J^{\prime \prime}\right) s^{2} G_{2}, \\
& \Delta \epsilon=(\epsilon-\delta \cos 2 \psi) \frac{J^{\prime \prime}}{J^{\prime}} s^{2} G_{2}, \\
& \Delta \Theta=-\sin \psi \cos \psi \frac{\delta}{\epsilon} \frac{J^{\prime \prime}}{J^{\prime}} s^{2} G_{2},
\end{aligned}
$$

where

$$
J^{\prime}=d J / d m^{2}, \quad J^{\prime \prime}=d J^{\prime} / d m^{2} .
$$

Note that the eror in intensity $\Delta J$ depends only on the second moment of the PSF $F_{2}=s^{2} G_{2}$, and is independent of the ellipticity and position-angle profiles of the galaxy, while the errors in ellipticity and position angle do depend on the ellipticities of the galaxy and PSF, and the position-angle difference between the two, as would be expected. It is important to note that for small enough $s$ the higher-order moments of the PSF are not important. These formulas are valid only if the surface brightness can be expanded into a power series. It is obvious that they are not correct for blurring effects on an intensity distribution with a singularity. However, even in that case they can be used to estimate the errors well outside the center of the galaxy, where the effects from the singularity can be ignored.

To exemplify the effects of the seeing, we assume that the intensity profiles near the centers of ellipticals can be approximated by a profile similar to the Hubble profile, 


$$
J\left(m^{2}\right)=\frac{J_{0}}{1+m^{2} / r_{\mathrm{c}}^{2}},
$$

where $r_{c}$ is the core radius at which the intensity is half the central value. The derivatives $J^{\prime}$ and $J^{\prime \prime}$ are

$$
J^{\prime}=-\frac{J_{0}}{r_{\mathrm{c}}^{2}}\left(\frac{J}{J_{0}}\right)^{2}, \quad J^{\prime \prime}=\frac{2 J_{0}}{r_{\mathrm{c}}^{4}}\left(\frac{J}{J_{0}}\right)^{3} .
$$

The change in surface brightness is

$$
\begin{aligned}
\Delta \frac{J}{J_{0}} & =\left(\frac{-1}{r_{\mathrm{c}}^{2}}+\frac{2 m^{2}}{r_{\mathrm{c}}^{4}} \frac{J}{J_{0}}\right)\left(\frac{J}{J_{0}}\right)^{2} F_{2} \\
& =\left(1-2 \frac{J}{J_{0}}\right)\left(\frac{J}{J_{0}}\right)^{2} \frac{F_{2}}{r_{\mathrm{c}}^{2}} .
\end{aligned}
$$

The change in ellipticity and position angle are

$$
\begin{aligned}
& \Delta \epsilon=-2(\epsilon-\delta \cos 2 \psi) \frac{J}{J_{0}} \frac{F_{2}}{r_{\mathrm{c}}^{2}}, \\
& \Delta \Theta=\sin 2 \psi \frac{\delta}{\epsilon} \frac{J}{J_{0}} \frac{F_{2}}{r_{\mathrm{c}}^{2}} .
\end{aligned}
$$

We define the apparent core radius $r_{\mathrm{c}, \text { app }}$ as the radius at which the observed surface brightness is half the central value. Without blurring, this radius is equal to the core radius $r_{\mathrm{c}}$ of the Hubble profile. We can estimate the measured core radius $r_{\mathrm{c} \text {,app }}$ as a function of $F_{2}$ by evaluation of $\Delta J$ at $r=0$ and at $r=r_{\mathrm{c}}$, and linearization of $J$ near $r=r_{\mathrm{c}}$. We obtain

$$
\frac{r_{\mathrm{c}, \mathrm{app}}}{r_{\mathrm{c}}}-1=\frac{F_{2}}{r_{\mathrm{c}}^{2}} \text {. }
$$

Formulas (17)-(19) are the expressions we want to use to estimate the errors in our data due to blurring. These formulas are valid to second order in $s$, and for profiles that are reasonably like the profiles used in Eq. (15).

It turns out that most of our galaxies were unresolved in the center, and hence the core radii could not be determined. In the limit of very small core radii, formulas (17)-(19) reduce to

$$
\begin{aligned}
\frac{\Delta J}{J} & =\frac{F_{2}}{r^{2}}, \\
\Delta \epsilon & =-(\epsilon-\delta \cos 2 \psi)\left(2 F_{2} / r^{2}\right), \\
\Delta \Theta & =\sin 2 \psi \frac{\delta}{\epsilon} \frac{F_{2}}{r^{2}} .
\end{aligned}
$$

It is obvious that the effects on ellipticity and position angle decrease at larger radii. The change in surface brightness $\Delta J$ decreases as $r^{-4}$ at large radii, which implies that the change in magnitude decreases as $r^{-2}$. It is easy to derive from Eq. (11) that this will be the case for any power law dependence of $J$ on $r$. Thus, the effects are minimal if we are far from the center; this is quantified below.

This conclusion relies on the convergence of the series in Eq. (A7) in Appendix A. We expect no problems if the PSF does not have strong wings that extend to very large radii. If, for example, the intensities in the PSF and the galaxy drop off like power laws at large radii, then the results will not hold if the PSF drops off less steeply than the galaxy intensity. In this case the contribution from the light of the center of the galaxy will exceed the local contribution to the measured intensity. With the PSFs for our data (see Fig. 3 below) and the galaxy profiles measured here, characterized by the model (15), this is not a concern, as noted by other authors (e.g., Capaccioli and de Vaucouleurs 1983).
The implication of these results is that the second-order moment of the PSF profile is the important parameter for determining the effect of seeing, and not the FWHM or the Gaussian $\sigma$ of the PSF. This becomes important when we want to compare data taken with different telescopes, which might have different PSFs. Schweizer $(1979,1981)$ has calculated numerically the seeing effects of various PSFs on the observed central surface brightness and core radius. $\mathrm{He}$ found that the effects could vary significantly for PSFs with the same FWHM but different wings. We have scaled Schweizer's PSFs and core radii such that the core radius of the galaxy was kept constant at unity. We have calculated the second-order moment of his PSFs, and in Fig. 2 we plot the change in central surface brightness $\Delta J(0) / J(0)$ as a function of the second-order moment $F_{2}$. Note how well the values lie along a curve. We have also drawn the line predicted by our second-order approximation. It clearly overestimates the effect of seeing when the core radius is small with respect to the FWHM of PSF, but gives a good approximation for small seeing effects in the regime where $\Delta J(0) /$ $J(0) \leqslant 0.3$.

Figure 3 shows the PSFs for the different telescopes from our data. Note the similarity between the profiles. We assume that the PSFs for our observations can be parametrized by one single function, and so we can use the FWHM of the PSF as a scale factor. For our mean PSF the moment $F_{2}$ is related to the FWHM by

$$
F_{2}=0.85(\text { FWHM })^{2} \text {, }
$$

which is a factor of 2.4 larger than what we would have for a Gaussian PSF. We have checked the analytical results numerically for a few cases, of which we show two in Fig. 4. Figures $4(a)-4$ (c) are a simulation of our highest-resolu-

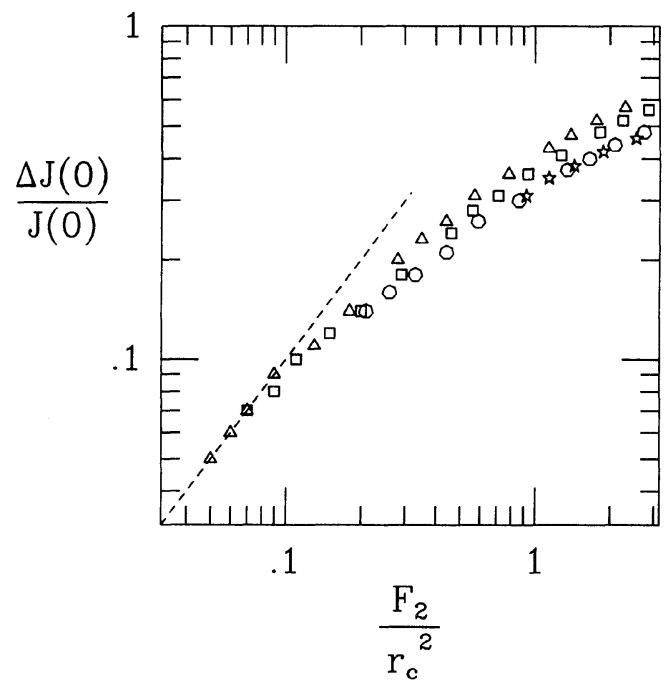

FIG. 2. Fractional change in central surface brightness $\Delta J(0) /$ $J(0)$ as calculated by Schweizer for a King model with concentration index $c=2.25$ versus the second-order moment of the PSF. The PSFs were normalized with respect to the core radius of the galaxy. The different symbols denote the PSFs with different wings. The triangles, squares, circles, and stars are Schweizer's profiles G, GE2, GE1, and GE.5, respectively. The dashed line is the analytically predicted second-order change in the central surface brightness. 


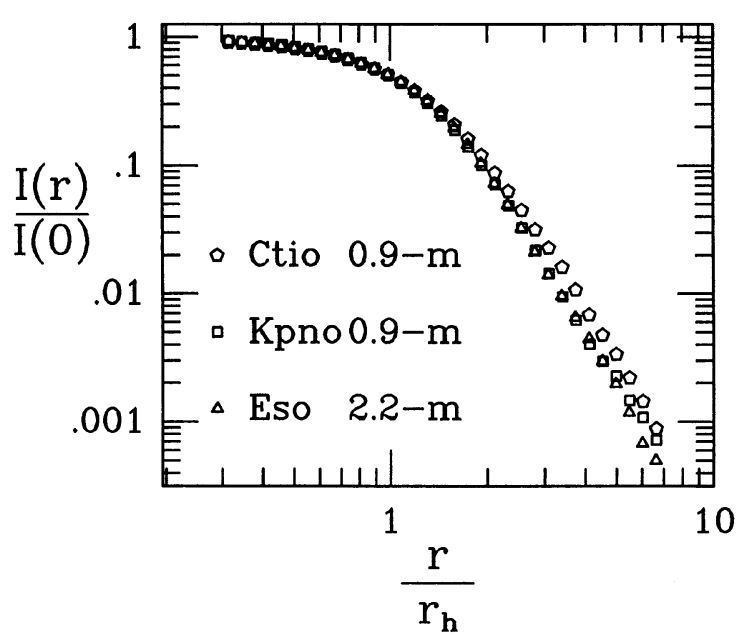

FIG. 3. PSFs from ESO, CTIO, and KNPO, all scaled to the same radius $r_{\mathrm{h}}$, at which the intensity is half the central intensity. The rapid decline in the seeing profile by three orders of magnitude over a factor of $10 \mathrm{in} \mathrm{radius}$ is noteworthy, as is the uniformity of the seeing profiles from different telescopes and sites.

tion exposure on the flattened elliptical NGC 1700. The unconvolved surface-brightness distribution has a core radius of 1 " and an ellipticity of 0.3 . The PSF is circularly symmetric here with a FWHM of 1.2". In Figs. 4(d)-4(f) we simulate NGC 7145, a very round elliptical. The unconvolved distribution has a core radius of 1 " and an ellipticity of 0.05 . The PSF is elliptical with an ellipticity $\delta=0.1$ and a FWHM of $1.5^{\prime \prime}$. Neither model had any change of position angle with radius. Note the drop in ellipticity in the center in both galaxies, and the dramatic change in the position angle in the round galaxy. The effects of the seeing extend to large radii, $\approx 5-10$ FWHM. One clearly needs to exercise care in interpreting structural changes near the centers of galaxies. We also show the analytical results from our second-order analysis, Eqs. (17)-(19). Clearly, they inadequately describe the effects near the center, since the core radius is significantly smaller than the seeing FWHM, but are quite adequate for establishing the point at which the data become reliable at the level of $10 \%$ or \pm a few degrees in position angle. These analytical approximations should not be used for correction of large seeing effects in the data, as noted above. Numerical techniques, or a more complete analytical analysis, are needed.

\section{RESULTS}

\section{a) Final Preparation of the Data}

We have calculated the radii at which the seeing changes the surface brightness by $0.05 \mathrm{mag}$, the ellipticity by 0.02 , and the position angle by $5^{\circ}$. For very round galaxies, i.e., for those with $\langle\epsilon\rangle \leqslant 0.08$, we chose an ellipticity cutoff of 0.01 . For the PSF we used a lower limit for the ellipticity $\delta$ equal to 0.1 . It proved difficult to measure $\delta$ from the generally low $\mathrm{S} / \mathrm{N}$ stellar objects in the galaxy frames, and so we assumed that $\delta=0.1$ would be a reasonable lower limit. We did not rely on the position angles measured for the PSF, but used a value of the angle $\psi$ of $45^{\circ}$ for the estimate of the effects on position angle [Eq. (23)], and simply used $\max (\epsilon, \delta)$ instead of $(\epsilon-\delta \cos 2 \psi)$ for the estimate of the seeing on ellip-

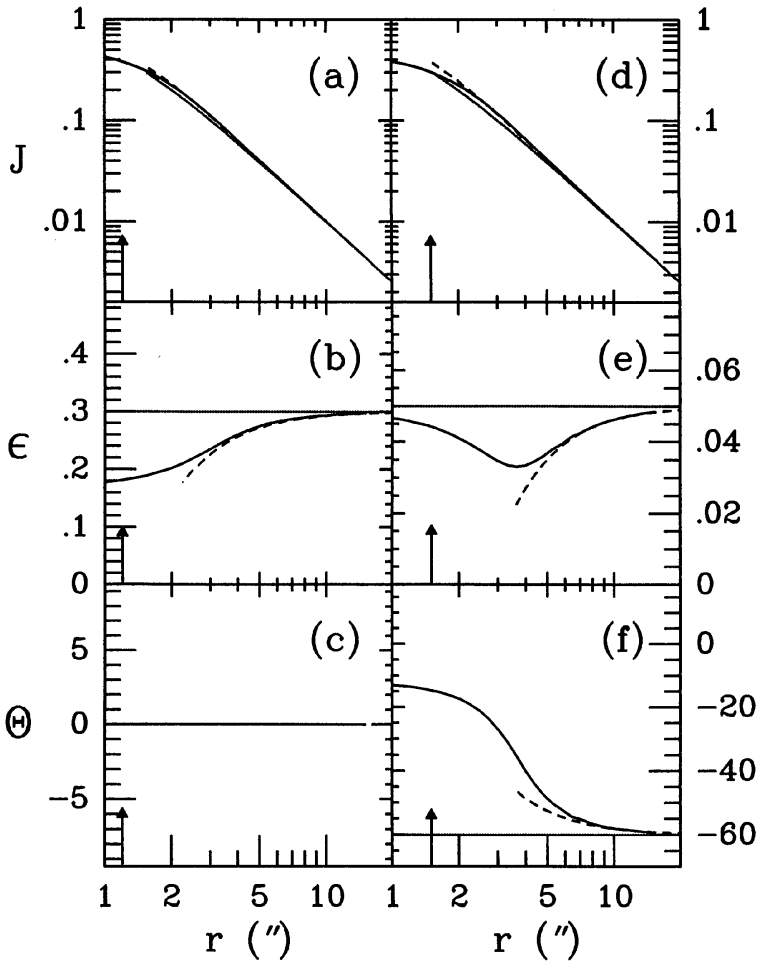

FIG. 4. PSF effects on the intensity, ellipticity, and position angle of a flat galaxy $(\mathrm{a}-\mathrm{c})$ and a round galaxy $(\mathrm{d}-\mathrm{f})$ that are similar to galaxies in our sample. The dotted line shows the original intensity, ellipticity, or position angle, the full line shows the numerical convolution of the seeing profile, and the dashed lines are the predicted values from Eqs. (17) -(19). The PSF was assumed to be Gaussian for ease of calculation, with a second moment equal to the second moment of the PSF of the observation. The values of the position angle and ellipticity are typical of NGC $1700(a-c)$ and NGC $7145(d-f)$. The PSF was circular for $(\mathrm{a}-\mathrm{c})$, and elliptical for $(\mathrm{d}-\mathrm{f})$, with an ellipticity $\delta=0.1$. For $(\mathrm{a}-\mathrm{c})$ the Gaussian PSF had a FWHM of 1.9", and had the same second-order moment as the seeing PSF of the $R$ exposure of NGC 1700 with a FWHM of 1.2". The profiles in (d-f) were calculated with a Gaussian PSF with a FWHM of $2.3^{\prime \prime}$. This PSF had the same second-order moment as the PSF of the $R$ exposure of NGC 7145, which had a FWHM of 1.5 ". The vertical arrows show the FWHM of the PSF of the observation.

ticity [Eq. (22)]. The inner cutoff radii calculated with these criteria are listed in Table III in columns 8-10. The data were $K$ corrected, and corrected for galactic absorption using the relations from Davis et al. (1985):

$$
\begin{aligned}
A_{v} & = \begin{cases}0 & |b|>50^{\circ} \\
0.1[1 / \sin (b)-1] & |b|<50^{\circ},\end{cases} \\
\delta U & =-4 z-1.56 A_{v}, \\
\delta B & =-5 z-1.33 A_{v}, \\
\delta R & =-z-0.74 A_{v},
\end{aligned}
$$

where $b$ is the galactic latitude and $z$ is the redshift $v / c$. The corrections for each galaxy are listed in Table V. In Figs. $9(a)-9$ (q) the resulting profiles are drawn, and the data are listed in Table IX in Appendix B, along with notes on the individual galaxies. While the full surface-brightness profile has been plotted, the ellipticity and position-angle profiles 
TABLE V. Average colors and gradients.

\begin{tabular}{|c|c|c|c|c|c|c|c|}
\hline $\begin{array}{l}\text { Galaxy } \\
\text { (1) }\end{array}$ & $\begin{array}{l}\delta U \\
(2)\end{array}$ & $\begin{array}{r}\delta B \\
(3)\end{array}$ & $\begin{array}{r}\delta R \\
(4)\end{array}$ & $\begin{array}{c}<B-R> \\
(5)\end{array}$ & $\begin{array}{c}<U-R> \\
\text { (6) }\end{array}$ & $\begin{array}{c}\frac{\Delta(B-R)}{\Delta \log r} \\
(7)\end{array}$ & $\begin{array}{c}\frac{\Delta(U-R)}{\Delta \log r} \\
(8)\end{array}$ \\
\hline NGC 636 & -0.025 & -0.031 & -0.006 & $1.76 \pm 0.00$ & $2.23 \pm 0.00$ & $-0.08 \pm 0.03$ & $-0.25 \pm 0.07$ \\
\hline NGC 1199 & -0.031 & -0.039 & -0.008 & $1.89 \pm 0.00$ & - & $-0.11 \pm 0.05$ & - \\
\hline NGC 1379 & -0.019 & -0.024 & -0.005 & $1.69 \pm 0.04$ & $2.09 \pm 0.12$ & $-0.06 \pm 0.08$ & $-0.12 \pm 0.24$ \\
\hline NGC 1395 & -0.021 & -0.026 & -0.005 & $1.86 \pm 0.01$ & $2.39 \pm 0.02$ & $-0.06 \pm 0.02$ & $-0.28 \pm 0.08$ \\
\hline NGC 1399 & -0.019 & -0.024 & -0.005 & $1.85 \pm 0.01$ & $2.40 \pm 0.10$ & $-0.06 \pm 0.02$ & $-0.14 \pm 0.21$ \\
\hline NGC 1404 & -0.019 & -0.024 & -0.005 & $1.82 \pm 0.00$ & $2.39 \pm 0.01$ & $-0.12 \pm 0.02$ & $-0.19 \pm 0.05$ \\
\hline NGC 1407 & -0.021 & -0.026 & -0.005 & $1.93 \pm 0.03$ & $2.57 \pm 0.12$ & $0.00 \pm 0.04$ & $-0.10 \pm 0.18$ \\
\hline NGC 1439 & -0.021 & -0.026 & -0.005 & $1.72 \pm 0.03$ & $2.07 \pm 0.10$ & $-0.08 \pm 0.07$ & $-0.43 \pm 0.40$ \\
\hline NGC 1549 & -0.084 & -0.078 & -0.036 & $1.72 \pm 0.01$ & $2.11 \pm 0.04$ & $-0.07 \pm 0.02$ & $-0.27 \pm 0.16$ \\
\hline NGC 1700 & -0.231 & -0.218 & -0.097 & $1.74 \pm 0.00$ & $2.25 \pm 0.02$ & $-0.07 \pm 0.02$ & $-0.32 \pm 0.08$ \\
\hline NGC 2986 & -0.257 & -0.230 & -0.115 & $1.79 \pm 0.02$ & $2.30 \pm 0.05$ & $-0.04 \pm 0.05$ & $-0.19 \pm 0.12$ \\
\hline NGC 7144 & -0.025 & -0.031 & -0.006 & $1.72 \pm 0.01$ & $2.17 \pm 0.06$ & $-0.01 \pm 0.09$ & $-0.33 \pm 0.15$ \\
\hline NGC 7145 & -0.025 & -0.031 & -0.006 & $1.66 \pm 0.02$ & $1.91 \pm 0.08$ & $-0.13 \pm 0.06$ & $-0.44 \pm 0.21$ \\
\hline IC 1459 & -0.022 & -0.027 & -0.005 & $1.78 \pm 0.02$ & - & $-0.13 \pm 0.04$ & - \\
\hline NGC 7507 & -0.021 & -0.026 & -0.005 & $1.88 \pm 0.01$ & $2.41 \pm 0.04$ & $-0.09 \pm 0.04$ & $-0.13 \pm 0.17$ \\
\hline NGC 7619 & -0.101 & -0.104 & -0.037 & $1.87 \pm 0.15$ & - & $0.03 \pm 0.71$ & - \\
\hline NGC 7626 & -0.101 & -0.104 & -0.037 & $1.85 \pm 0.02$ & $2.44 \pm 0.08$ & $-0.11 \pm 0.10$ & $-0.09 \pm 0.49$ \\
\hline
\end{tabular}

Notes: Columns (2), (3), (4) give the combination of the $\mathrm{K}$ correction and correction for galactic absorption for each band. Columns (5) and (6) give the average $B-R$ and $U-R$ color at $r_{e} / 2$ from the $r_{e}$ values in Table I. Columns (7) and (8) give the logarithmic color gradients. The colors and the gradients are determined from a fit to the data in the radial interval of $5^{\prime \prime}$ to $30^{\prime \prime}$. The errors for the gradients incorporate possible systematic effects due to uncertainties in the background determination.

have been cut off in the centers at the radii tabulated in Table III. The uncertainty due to the background is represented by the error bars at the outer ends of the surface-brightness profiles. The color gradients are cut off at a radius of 50", which was the inner radius of the interval that we used for the background fitting. For each band, the radius at which the seeing effects were 0.05 mag was calculated. The cutoff radius for the color profile was the larger of the two cutoff radii for each of the bands involved. The errors in the color profiles incorporate errors due to noise as well as the systematic errors due to background subtraction, with the latter being dominant at large radii. The error in the color from uncertainty in the background was taken to be half of the square root of the sum of the squared error estimates of the separate profiles. This correction by a factor of $\frac{1}{2}$ was based on the results of the internal comparison of the color profiles, which suggested that the errors in the background determination were in the same sense for all bandpasses, and therefore partly cancelled in the color profile. We note that for the great majority of galaxies, the color profiles are very regular, and do not show any strong changes in slope as expected for gradients that result from large errors in the background level. This confirms that the systematic uncertainty due to the background determination is of comparable sense in both bandpasses used to derive the color profile. The plotted errors may still be too large. However, it is very difficult to establish the magnitude of this overestimation, and thus the confidence level for the observed gradients. An external comparison (below) with the work of others gives us some added confidence that our gradients are of the right sense and magnitude.

\section{b) Average Values}

We have determined the global properties of the surface brightness and structural profiles and their mean values, and listed them in Tables V, VI, and VII. In Table V we give mean color at $r_{\mathrm{e}} / 2$, and the logarithmic color gradients $(\Delta C / \Delta \log r)$. The mean and the gradient were determined from fitting a line to the colors as a function of $\log r$ over the interval of $5^{\prime \prime}-30$ ". The mean ellipticity and position angle at $r_{\mathrm{e}} / 2$ were derived from the data in the interval of $10^{\prime \prime}-40^{\prime \prime}$, and tabulated in Table VI, as were their logarithmic gradients $\Delta \epsilon / \Delta \log r$ and $\Delta \Theta / \Delta \log r$ over the same radius range. These radial intervals were chosen because of the influence of systematic effects and noise both at small radii $\left(<5^{\prime \prime}\right)$ and large radii ( $>50^{\prime \prime}$ ) on the observed profiles, and are not related to any scale length of the galaxy. We first determined the $B$ and $R$ ellipticities separately, but found no significant difference for these galaxies. The $R$ data values are tabulated in Table VI. In Table VII(a) we present mean high-order $(3 \phi$ and $4 \phi$ ) deviations from ellipses. We have median fil- 
TABLE VI. Average ellipticity, position angle, and their gradients.

\begin{tabular}{|c|c|c|c|c|c|}
\hline $\begin{array}{l}\text { Galaxy } \\
\text { (1) }\end{array}$ & $\begin{array}{c}<\epsilon> \\
(2)\end{array}$ & $\begin{array}{c}\langle\theta> \\
(3)\end{array}$ & $\begin{array}{c}\Delta \epsilon / \Delta \log r \\
(4)\end{array}$ & $\begin{array}{c}\Delta \theta^{-} \Delta \mathrm{l} \text { lc } \\
(5)\end{array}$ & $\log r$ \\
\hline NGC 636 & $0.14 \pm 0.01$ & $69.2 \pm 1.0$ & $0.06 \pm 0.02$ & $-52.6 \pm$ & 2.7 \\
\hline NGC 1199 & $0.25 \pm 0.00$ & $50.6 \pm 0.2$ & $-0.11 \pm 0.01$ & $4.6 \pm$ & 1.0 \\
\hline NGC 1379 & $0.03 \pm 0.00$ & $6.8 \pm 1.5$ & $0.00 \pm 0.00$ & $20.8 \pm$ & 8.8 \\
\hline NGC 1395 & $0.18 \pm 0.00$ & $94.8 \pm 0.1$ & $0.08 \pm 0.01$ & $13.4 \pm$ & 0.8 \\
\hline NGC 1399 & $0.11 \pm 0.00$ & $-70.6 \pm 0.2$ & $-0.05 \pm 0.00$ & $-10.2 \pm$ & 1.2 \\
\hline NGC 1404 & $0.13 \pm 0.00$ & $-19.3 \pm 0.2$ & $-0.03 \pm 0.01$ & $13.1 \pm$ & 0.7 \\
\hline NGC 1407 & $0.05 \pm 0.00$ & $55.2 \pm 0.5$ & $-0.01 \pm 0.00$ & $3.7 \pm$ & 1.6 \\
\hline NGC 1439 & $0.09 \pm 0.00$ & $33.8 \pm 0.4$ & $0.02 \pm 0.01$ & $3.7 \pm$ & 2.2 \\
\hline NGC 1549 & $0.13 \pm 0.00$ & $-55.9 \pm 0.3$ & $-0.01 \pm 0.01$ & $53.8 \pm$ & 1.8 \\
\hline NGC 1700 & $0.25 \pm 0.00$ & $88.2 \pm 0.6$ & $0.07 \pm 0.01$ & $3.1 \pm$ & 1.2 \\
\hline NGC 2986 & $0.14 \pm 0.00$ & $34.1 \pm 0.1$ & $0.00 \pm 0.01$ & $7.1 \pm$ & 0.7 \\
\hline NGC 7144 & $0.03 \pm 0.00$ & $19.6 \pm 0.7$ & $0.01 \pm 0.00$ & $-53.6 \pm$ & 4.0 \\
\hline NGC 7145 & $0.05 \pm 0.00$ & $-51.9 \pm 2.2$ & $-0.04 \pm 0.02$ & $78.3 \pm 1$ & 13.4 \\
\hline IC 1459 & $0.26 \pm 0.00$ & $38.1 \pm 0.1$ & $0.00 \pm 0.01$ & $13.3 \pm$ & 0.4 \\
\hline NGC 7507 & $0.04 \pm 0.00$ & $-81.8 \pm 1.0$ & $0.01 \pm 0.01$ & $2.6 \pm$ & 5.0 \\
\hline NGC 7619 & $0.24 \pm 0.00$ & $35.2 \pm 0.3$ & $-0.15 \pm 0.01$ & $3.0 \pm$ & 1.5 \\
\hline NGC 7626 & $0.13 \pm 0.00$ & $12.3 \pm 0.3$ & $0.13 \pm 0.00$ & $4.3 \pm$ & 1.6 \\
\hline
\end{tabular}

Notes: Columns (2) and (3) give the mean ellipticity and position angle at $r_{e} / 2$ determined from the $R$-band data in the interval of $10^{\prime \prime}$ to $40^{\prime \prime}$. Columns (4) and (5) give the logarithmic gradients. The errors are the formal errors from the fit. Systematic errors will generally be larger than the formal errors for the mean ellipticity and position angle.

tered the profiles of the residual $\cos (n \phi)$ and $\sin (n \phi)$ terms with a five-point running filter, and determined the amplitudes and phases (see subsec. $d$ ). The maxima and radii of the maxima are listed in Table VIII(b) for the $3 \phi$ and $4 \phi$ amplitudes.

\section{c) Literature Comparisons}

The data are compared to similar CCD data available in the literature, from Kent (1984), Lauer (1985a), Djorgovski (1985), Jedrzejewski (1987), Capaccioli et al. (1988), and Peletier et al. (1989). Our surface-brightness profiles were differenced point by point, interpolating as necessary, with those of the other authors and plotted as (us - others) as a function of radius in Fig. 5. The differences are tabulated in Table VIII. The logarithmic gradient (e.g., $\Delta R / \Delta \log r$ for the $R$ band) and the rms deviation of the difference profile was derived by fitting over the radius range $5^{\prime \prime}-30$ ". We ignored deviations in the zero points, because they were mainly due to differences in the $R$ band photometric system used by the different authors. The agreement for the ellipticity and position-angle profiles is good. We obtain an rms mean ellipticity difference of 0.02 and an rms mean position-angle difference of $2^{\circ}$ with all the above authors over the radius range $10^{\prime \prime}-40^{\prime \prime}$, where seeing effects do not play a role.

The surface-brightness-profile differences are larger than one might initially expect. The observations by Lauer and Djorgovski had an even smaller field of view than most of our data. The resulting background uncertainty is probably the reason for the differences seen in Table VIII. Djorgovski included these uncertainties in his listed errors, and we note that his errors are realistic in most cases. We find a mean absolute difference in the radial gradient of 0.09 mag per decade in radius for Djorgovski and 0.14 for Lauer. The agreement with Jedrzejewski and Peletier et al. is better. The $B$ band data from Jedrzejewski compare very well with ours, but the $R$ band data give a residual gradient for two of the three galaxies in common, an effect that was also noted by Peletier et al. for the ellipticals common to both authors. For the three galaxies in common in Table VIII, Jedrzejewski's $B-R$ gradient differs by 0.07 , in the sense that our gradient is steeper. The rms gradient of the difference profile with Peletier et al. is only 0.04 mag per decade, within the range expected from our background uncertainty. However, we only have two galaxies and three useful profiles in common (our NGC $7619 B$ frame suffered from an extremely bright sky and is thus very uncertain). The current surface-brightness profiles agree at the level of $\approx 0.1 \mathrm{mag}$ per decade, with the newest and larger field data agreeing to probably a factor of 2 better at $\approx 0.05$ mag per decade in radius. Since most of this difference can probably be attributed to uncertainty in estimating the background, we expect that future data will show better agreement as larger CCDs are implemented.

Taken at face value, these results and the error estimates noted above would lead one to be concerned about the $B-R$ color gradients which show, in the mean for our sample, gradients of the order of $0.1 \mathrm{mag}$ per decade. However, as we also noted above, we expect that some of the systematic errors in estimating the background are common to both col- 
TABLE VII(a). Average residuals.

\begin{tabular}{|c|c|c|c|c|c|c|c|c|}
\hline & $\left\langle C_{3}\right\rangle$ & $\begin{array}{r}\left\langle S_{3}\right\rangle \\
10^{\prime \prime}<\end{array}$ & $\begin{array}{c}\left\langle C_{4}\right\rangle \\
<<4\end{array}$ & $\left\langle S_{4}\right\rangle$ & $\begin{array}{r}\left\langle C_{3}\right\rangle \\
0\end{array}$ & & & $\begin{array}{l}\left\langle S_{4}\right\rangle \\
r_{e}\end{array}$ \\
\hline (1) & (2) & (3) & (4) & (5) & (6) & (7) & (8) & (9) \\
\hline NGC 636 & -0.1 & 0.1 & 0.4 & 0.5 & -0.1 & 0.3 & 0.7 & 0.6 \\
\hline NGC 1199 & -0.3 & 0.0 & -0.7 & 0.2 & -0.4 & 0.0 & -0.1 & 0.3 \\
\hline NGC 1379 & 0.0 & 0.0 & 0.1 & -0.1 & 0.1 & 0.1 & 0.1 & -0.1 \\
\hline NGC 1395 & 0.0 & -0.1 & -0.1 & -0.5 & 0.1 & -0.3 & -0.7 & -0.6 \\
\hline NGC 1399 & -0.1 & -0.1 & 0.0 & -0.1 & 0.0 & 0.0 & 0.3 & 0.0 \\
\hline NGC 1404 & 0.0 & 0.0 & 0.1 & -0.2 & 0.0 & 0.0 & -0.2 & -0.2 \\
\hline NGC 1407 & 0.0 & 0.0 & -0.2 & 0.1 & 0.0 & -0.2 & 0.0 & 0.4 \\
\hline NGC 1439 & 0.0 & -0.1 & -0.1 & -0.2 & 0.2 & -0.2 & -0.1 & -0.2 \\
\hline NGC 1549 & 0.1 & -0.1 & -0.3 & 0.2 & 0.1 & -0.3 & 0.4 & 1.3 \\
\hline NGC 1700 & 0.1 & -0.2 & 0.9 & 0.5 & 0.0 & -0.1 & 1.1 & 0.3 \\
\hline NGC 2986 & 0.1 & -0.1 & -0.2 & -0.1 & 0.1 & -0.6 & -0.4 & 0.0 \\
\hline NGC 7144 & 0.0 & -0.1 & 0.0 & -0.1 & 0.1 & 0.1 & 0.0 & 0.0 \\
\hline NGC 7145 & 0.0 & 0.0 & 0.0 & -0.1 & 0.0 & 0.0 & -0.3 & -0.1 \\
\hline IC 1459 & 0.1 & 0.1 & 0.1 & 0.0 & 0.3 & 0.2 & -0.2 & 0.5 \\
\hline NGC 7507 & 0.0 & 0.1 & 0.1 & -0.1 & 0.0 & 0.1 & 0.1 & -0.1 \\
\hline NGC 7619 & -0.2 & -0.3 & 0.1 & 0.0 & -0.5 & -0.4 & 0.2 & -0.1 \\
\hline NGC 7626 & -0.2 & -0.3 & -0.1 & 0.7 & -0.6 & -0.9 & 0.2 & 0.9 \\
\hline
\end{tabular}

Notes: The amplitude of the residuals are given $\times 100$ (i.e., as percentages). The peak $C_{4}$ in NGC 1700 is $0.05(5 \%)$.

TABLE VII(b). Maximum residual amplitudes.

\begin{tabular}{|c|c|c|c|c|c|c|c|c|c|c|c|c|}
\hline Galaxy & $r$ & $A_{3}$ & $\begin{array}{c}\Theta_{3} \\
\operatorname{Max}\end{array}$ & $\begin{array}{c}r \\
\text { mum }\end{array}$ & $A_{4}$ & $\Theta_{4}$ & & & $\begin{array}{l}\Theta_{3} \\
\text { ond }\end{array}$ & $\stackrel{r}{\mathrm{Ma}}$ & $\begin{array}{c}A_{4} \\
\text { cimum }\end{array}$ & $\Theta_{4}$ \\
\hline (1) & (2) & (3) & (4) & (5) & (6) & (7) & (8) & (9) & (10) & (11) & (12) & (13) \\
\hline NGC 636 & 41 & 0.8 & 82 & 37 & 1.0 & 26 & - & - & - & - & - & - \\
\hline NGC 1199 & 19 & 0.6 & 62 & 13 & 1.2 & 43 & 60 & 1.1 & 75 & 60 & 2.1 & 84 \\
\hline NGC 1379 & 45 & 0.3 & 11 & 26 & 0.3 & 77 & - & - & - & - & - & - \\
\hline NGC 1395 & 66 & 0.4 & 103 & 66 & 1.4 & 48 & - & - & - & - & - & - \\
\hline NGC 1399 & 23 & 0.2 & 80 & 28 & 0.3 & 86 & - & - & - & - & - & - \\
\hline NGC 1404 & 45 & 0.1 & 67 & 8 & 0.6 & 6 & - & - & - & - & - & - \\
\hline NGC 1407 & 80 & 0.6 & 78 & 37 & 0.6 & 23 & - & - & - & - & - & - \\
\hline NGC 1439 & 26 & 0.4 & 24 & 21 & 0.5 & 58 & - & - & - & - & - & - \\
\hline NGC 1549 & 28 & 0.5 & 106 & 45 & 1.5 & 18 & - & - & - & - & - & - \\
\hline NGC 1700 & 21 & 0.4 & 103 & 8 & 1.4 & 88 & 55 & 1.7 & 63 & 55 & 3.4 & 35 \\
\hline NGC 2986 & 34 & 0.5 & 98 & 34 & 0.5 & 45 & - & - & - & - & - & - \\
\hline NGC 7144 & 26 & 0.3 & 112 & 23 & 0.3 & 84 & - & - & - & - & - & - \\
\hline NGC 7145 & 7 & 0.4 & 49 & 23 & 0.4 & 64 & - & - & - & - & - & - \\
\hline IC 1459 & 3 & 0.2 & 86 & 3 & 0.6 & 8 & 45 & 0.5 & 9 & 41 & 0.7 & 29 \\
\hline NGC 7507 & 17 & 0.4 & 17 & 21 & 0.3 & 41 & - & - & - & - & - & - \\
\hline NGC 7619 & 41 & 0.9 & 66 & 55 & 0.8 & 74 & - & - & - & - & - & - \\
\hline NGC 7626 & 31 & 1.2 & 77 & 31 & 1.1 & 23 & - & - & - & - & - & - \\
\hline
\end{tabular}

Notes: The amplitudes are given $\times 100$ (i.e., as percentages). The maximum amplitudes were determined from the median-filtered profiles of the high-order residuals. For 3 galaxies, two distinct maxima are listed. The phases are taken with respect to the major axis. The peak value of $A_{4}$ for the structure in NGC 1700 at $\mathrm{r}=55^{\prime \prime}$ is $0.05(5 \%)$. 


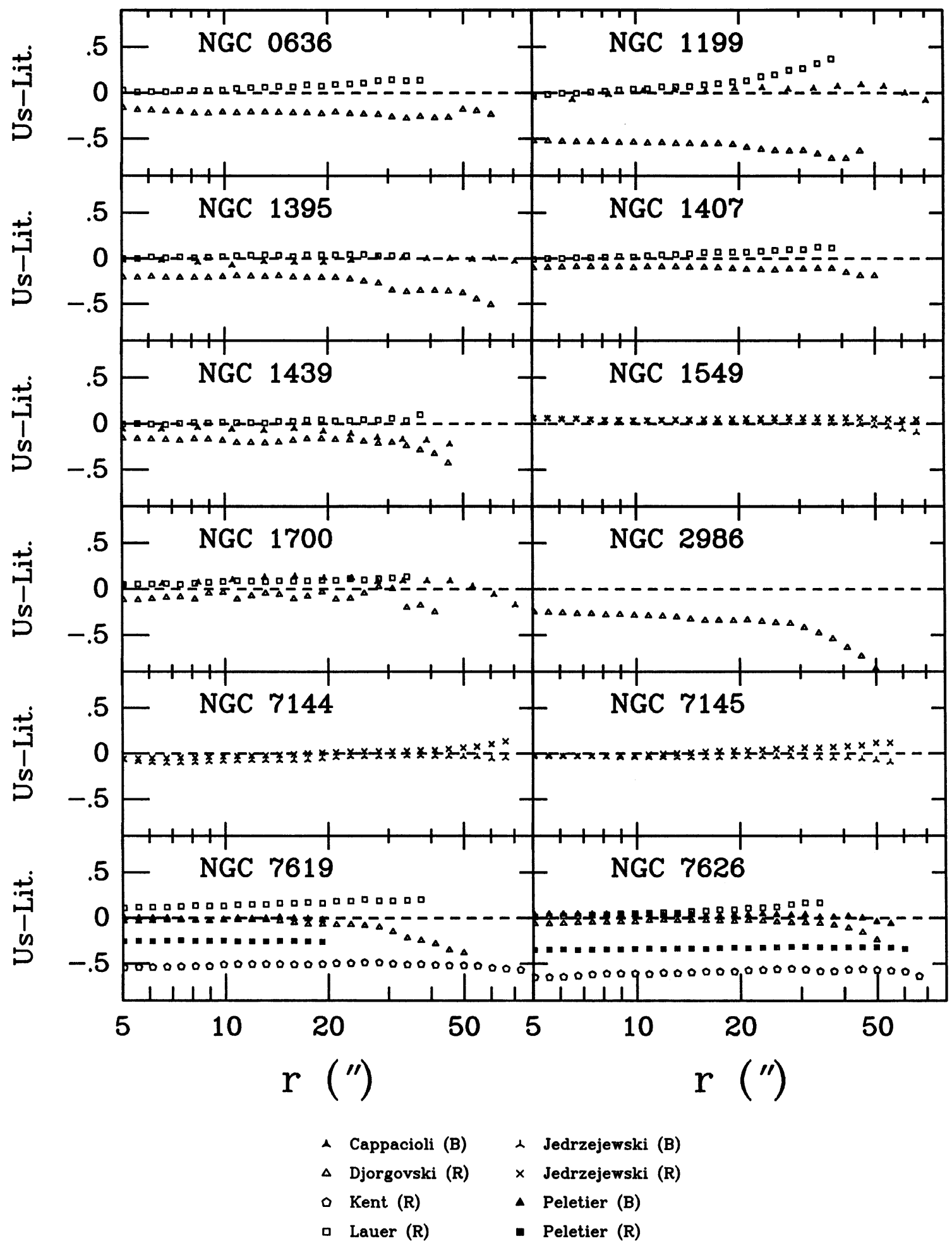

Fig. 5. Difference profiles of our data - literature data in magnitudes. 
TABLE VIII. Comparison with literature.

\begin{tabular}{|c|c|c|c|c|c|c|c|c|}
\hline \multirow{2}{*}{$\begin{array}{l}\text { Galaxy } \\
\text { (1) }\end{array}$} & \multirow{2}{*}{$\begin{array}{c}\text { Djor. } \\
\frac{\Delta R}{\Delta \log r} \\
(2)\end{array}$} & \multirow{2}{*}{$\begin{array}{c}\text { Lauer } \\
\frac{\Delta R}{\Delta \log r} \\
(3)\end{array}$} & \multirow{2}{*}{$\begin{array}{c}\text { Kent } \\
\frac{\Delta R}{\Delta \log r} \\
(4)\end{array}$} & \multirow{2}{*}{$\begin{array}{l}\text { Capa. } \\
\frac{\Delta B}{\Delta \log r} \\
(5)\end{array}$} & \multicolumn{2}{|c|}{ Jedrzejewski } & \multicolumn{2}{|c|}{ Peletier } \\
\hline & & & & & $\frac{\Delta B}{\Delta \log r}$ & $\begin{array}{c}\frac{\Delta R}{\Delta \log r} \\
(7)\end{array}$ & $\begin{array}{c}\frac{\Delta B}{\Delta \log r} \\
(8)\end{array}$ & $\begin{array}{c}\frac{\Delta R}{\Delta \log r} \\
(9)\end{array}$ \\
\hline NGC 636 & $-6 \pm 7$ & $15 \pm 4$ & & & & & & \\
\hline NGC 1199 & $-13 \pm 22$ & $32 \pm 2$ & & $16 \pm 3$ & & & & \\
\hline NGC 1395 & $-5 \pm 4$ & $5 \pm 3$ & & $3 \pm 2$ & & & & \\
\hline NGC 1407 & $-3 \pm 3$ & $14 \pm 3$ & & & & & & \\
\hline NGC 1439 & $-3 \pm 8$ & $7 \pm 5$ & & $-10 \pm 6$ & & & & \\
\hline NGC 1549 & & & & & $-5 \pm 2$ & $1 \pm 3$ & & \\
\hline NGC 1700 & $9 \pm 8$ & $8 \pm 3$ & & $8 \pm 2$ & & & & \\
\hline NGC 2986 & $-17 \pm 4$ & & & & & & & \\
\hline NGC 7144 & & & & & $9 \pm 4$ & $12 \pm 5$ & & \\
\hline NGC 7145 & & & & & $-1 \pm 5$ & $12 \pm 6$ & & \\
\hline NGC 7619 & $-13 \pm 7$ & $12 \pm 3$ & $7 \pm 3$ & & & & & $-5 \pm 3$ \\
\hline NGC 7626 & & $16 \pm 6$ & $12 \pm 6$ & & & & $-1 \pm 4$ & $4 \pm 6$ \\
\hline$\left\langle\frac{A b s(\Delta)}{\sigma}\right\rangle$ & 1.4 & 4.6 & 2.1 & 3.1 & 1.6 & 1.6 & .9 & \\
\hline$\langle\sigma\rangle$ & 8 & 4 & 5 & 3 & 4 & 5 & 4 & \\
\hline Median $\Delta$ & -5 & 14 & & 5 & -1 & 12 & -1 & \\
\hline$\langle\Delta\rangle$ & -6 & 15 & 10 & 4 & 1 & 8 & -1 & \\
\hline
\end{tabular}

Notes: Column (2) to (9) give the mean logarithmic gradients of the point-to-point differences $\left(\Delta\right.$ (mag $_{u s}$ -

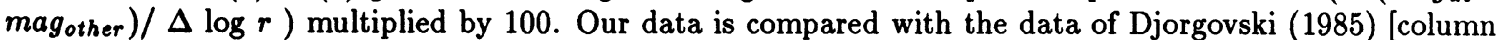
(2)], Lauer (1985a) [column (3)], Kent (1984) [column (4)], Capaccioli et al. (1988) [column (5)], Jedrzejewski (1987) [column (6) and (7)], and Peletier et al. (1989) [column (8) and (9)]. The errors reflect the uncertainty of our background determination and that of the literature, if provided. At the bottom, we give the mean ratio of the absolute difference gradient divided by the expected error, the mean expected error, the median difference gradient, and the mean difference gradient.

ors, and so the $B-R$ gradients may be more reliable than the errors would suggest. It is interesting to note that other authors (see, e.g., Boroson et al. 1983, 1987; Davis et al. 1985; Peletier et al. 1989) find $B-R$ color gradients that are similar in sense and magnitude in the mean, even though they too have expressed concern about the effect of the uncertain background subtraction. Independent confirmation of these color gradients comes from Frogel et al. (1978) and Sandage and Visvanathan (1978). The latter authors found an average gradient $\Delta(U-R) / \Delta \log r=-0.24$. The consistency of these results leads us to believe that the mean $U-R$ color gradient of -0.23 mag per decade is real, and that the $B-R$ gradient of -0.07 mag per decade is likely to be correct.

\section{d) High-Order Residuals}

It is interesting to note that many of the galaxies studied show residual harmonics $\sin (n \phi)$ or $\cos (n \phi)$, where $n=3,4$, with an amplitude larger than $0.5 \%$ at some radius. The only galaxies without any significant residuals throughout the observed radial range are NGC 1379 and NGC 7144, both very round galaxies. It is not clear whether the residuals in NGC 7507 and NGC 7145 (below 0.4\%) are real. Jedrzejewski (1987) and Peletier et al. (1989) determined residu- als for a large sample of galaxies. The comparison of our residuals with theirs for the galaxies in common showed good agreement; the differences are mostly within the errors for the residual terms, i.e., typically $0.2 \%$ to $0.3 \%$. Small systematic differences sometimes occur on a similar level. Bender, Döbereiner, and Möllenhof (1988) give the $\cos (3 \phi)$ and $\cos (4 \phi)$ terms of five galaxies, which are also consistent with ours. This confirms the reality of these structures and shows that they are not due to detector or calibration problems.

The standard interpretation of the residual $\cos (4 \phi)$ terms is that they indicate the presence of a weak disk (e.g., Lauer 1985b; Jedrzejewski 1987; Bender and Möllenhof 1987) when positive, and indicate boxiness caused possibly by an overpopulation of particular tube orbits around the rotation axis (e.g., Binney and Petrou 1985). The correlations between rotational velocity and $\cos (4 \phi)$ terms found by Carter (1987) and Bender (1988) lend support to such an interpretation. However, we wish to emphasize that the signs of the $\cos (4 \phi)$ and $\sin (4 \phi)$ terms are determined by the phase of the fourth-order residual with respect to the apparent long axis. Projection effects will cause position-angle differences between the projected disk and the main body of a triaxial galaxy (e.g., de Zeeuw and Franx 1989), thereby producing a phase shift of the residual harmonical terms. If such projec- 
tion effects are important, then we expect to see significant $\sin (4 \phi)$ terms. It is remarkable that no authors except Peletier et al. have presented the $\sin (n \phi)$ terms; for some galaxies they are similar to or stronger than the $\cos (n \phi)$ terms.

In total, six galaxies have significant $\sin (4 \phi)$ terms. We have plotted the residual terms of NGC 636, NGC 1199 , NGC 1395, NGC 1549, and NGC 1700 in various representations in Figs. 6 and 7. In Fig. 6, the residuals are expressed in $\cos (n \phi)$ and $\sin (n \phi)$, where $\phi$ is taken with respect to the north instead of the major axis of the galaxy. In Fig. 7, we plot the amplitudes of the residual terms $A_{3}$ and $A_{4}$ defined by

$$
A_{n}=\sqrt{\mathrm{C}_{n}^{2}+\mathrm{S}_{n}^{2}}
$$

and their phases $\Theta_{3}$ and $\Theta_{4}$, which are given by

$$
\sin \left(n \Theta_{n}\right)=S_{n} / A_{n}, \quad \cos \left(n \Theta_{n}\right)=C_{n} / A_{n},
$$

where $C_{n}$ and $S_{n}$ are the residual $\cos (n \phi)$ and $\sin (n \phi)$ terms. In Fig. 7 we have drawn the phases $\Theta_{n}$ with respect to the major axis and with respect to the north. We see that for NGC 636, NGC 1395, and NGC 1549 the phase changes of the residual fourth-order terms disappear when the phase is plotted with respect to the north instead of the major axis.

The galaxy NGC 1549 is one of the most "extreme" in its fourth-order residuals. At small radii, the $\cos (4 \phi)$ terms are negative, with a minimum of about $-0.5 \%$, they change sign at a radius of $30^{\prime \prime}$, and have an amplitude of about $+1 \%$ at larger radii. The $\sin (4 \phi)$ terms increase slowly with radius. This galaxy shows a large position-angle twist; the phase changes between the $\sin (4 \phi)$ and $\cos (4 \phi)$ terms could be at least partly related to this shift. This can clearly be seen in Figs. 6 and 7, where the phases of the $\cos (4 \phi)$ and $\sin (4 \phi)$ terms are very stable when plotted with respect to a fixed position angle (north). We may therefore interpret these residuals as the result of a superposition of two components, of which the brighter one has a constant position angle. This example shows that the interpretation of the signs of the residuals is not straightforward, in general, and especially in the case of a galaxy with a large position-angle twist.

NGC 1700 has the largest residuals in this sample. In the center, we see small positive $\cos (4 \phi)$ residuals. At $50^{\prime \prime}$, the position angle starts to change, and the fourth-order harmonical residuals start to rise as well, reaching a maximum of $5 \%$ at $70^{\prime \prime}$. A contour plot (Fig. 8) reveals that the contours are square at this radius. This feature is actually visible on the Palomar Sky Survey. It is surprising to see that again the phases of the $\cos (4 \phi)$ and $\sin (4 \phi)$ terms are almost constant when plotted relative to the north, instead of to the major axis. This behavior can be modeled by the superposition of a galaxy with constant position angle at $90^{\circ}$ and a ring-or disk-like component at an angle of $40^{\circ}$ from the major axis of the galaxy. We fixed the position angle of the galaxy at $90^{\circ}$, and ran the profile-fitting program again. The residuals of the fit showed the second component clearly. We determined its surface brightness by averaging the residual intensities at a radius of $70^{\prime \prime}$. We found colors $(B-R)_{\text {ring }}=1.45 \pm 0.05, \quad(U-R)_{\text {ring }}=1.82 \pm 0.2$, which were similar to the colors of the main galaxy, $(B-R)_{\text {gal }}=1.6$ and $(U-R)_{\text {gal }}=1.8$ at $50^{\prime \prime}$. This suggests that the stellar populations are comparable. Whether the ringlike component is a relic of galaxy formation or the result of a subsequent interaction is unclear.

We would inject an element of caution here by noting that no models of triaxial galaxies have been published yet in which the effects of large apparent position-angle twists on the isophotal shapes are evaluated. Hence we cannot exclude the possibility that the effects described above may be partly due to the position-angle twist. The above example does show, however, that we have to be careful when interpreting the fourth-order residuals. A superposition of a disk or a ring on an elliptical galaxy can give either positive or negative $\cos (4 \phi)$ and/or $\sin (4 \phi)$ terms, depending on the apparent orientation of the disk with respect to the apparent major axis of the galaxy. If galaxies are triaxial, then the subcomponent can appear to be misaligned merely by projection effects (see, e.g., de Zeeuw and Franx 1989). Hence, a superposition of a disk can produce boxlike distortions.

We note that seven galaxies of our sample of 17 galaxies show residual third-order harmonical terms on the order of $0.5 \%$ or higher. The galaxies discussed below with known dust absorption belong to these seven. The deviations from ellipses occur generally at radii larger than $30^{\prime \prime}$. These are possible due to dust, but may also reflect real distortions in the galaxies.

\section{e) Dust Absorption}

It is established that a considerable fraction $(20 \%-40 \%)$ of ellipticals show signs of dust absorption (e.g., Ebneter and Balick 1985; Lauer 1985b; Sadler and Gerhard 1985; Sparks et al. 1985; Ebneter, Djorgovski, and Davis 1988). A large fraction of the galaxies of our sample has been searched for dust absorption by these authors. The galaxy NGC 1199 has a well-known dust lane near the center. It is clearly visible in an image that is the difference of the original data frame and one generated using the parameters from the ellipse fitting. The dust absorption causes the negative $\cos (4 \phi)$ residuals at radii smaller than 30" (another example where the interpretation of these terms is not "standard"). At larger radii, the $\cos (4 \phi)$ terms are positive, possibly indicating the presence of a weak disk. At large radii, the third-order terms may be significant, but this is not certain because of the low surface brightness of the galaxy. The galaxy IC 1459 has a slightly asymmetric color image. This was found previously by Sparks et al. (1985). In the case of NGC 7507, we found a shift in the center of the ellipses which was different in $B$ and $R$. This subtle effect shows up in the color image after heavy smoothing, and it is probably real because Sparks et al. (1985) find the same asymmetry in their color map of the galaxy. None of the other galaxies in our sample showed similar evidence of dust absorption.

\section{DISCUSSION}

The quality of two-dimensional surface photometry from CCD data generally appears to be high, with the major remaining problem being uncertainty in the background ("sky") level on these small-format devices. While this makes it difficult to determine color gradients with a high level of confidence, particularly in $B-R$, other structural characteristics appear to be well determined-with one further caveat concerning the seeing. The sensitivity of the ellipticity and position-angle profiles to the seeing, even at radii much larger than the FWHM of the seeing PSF, was a striking result of an analytical derivation of the effects of seeing. The ellipticity decreases by $10 \%$ even at radii of five times the FWHM of the seeing PSF. Furthermore, for very round galaxies the position angles in the inner regions will be seriously perturbed by even quite small noncircularities in the seeing PSF. For example, an elliptical with an ellipticity of 0.05 developed $5^{\circ}$ of position-angle change at 5 times the 

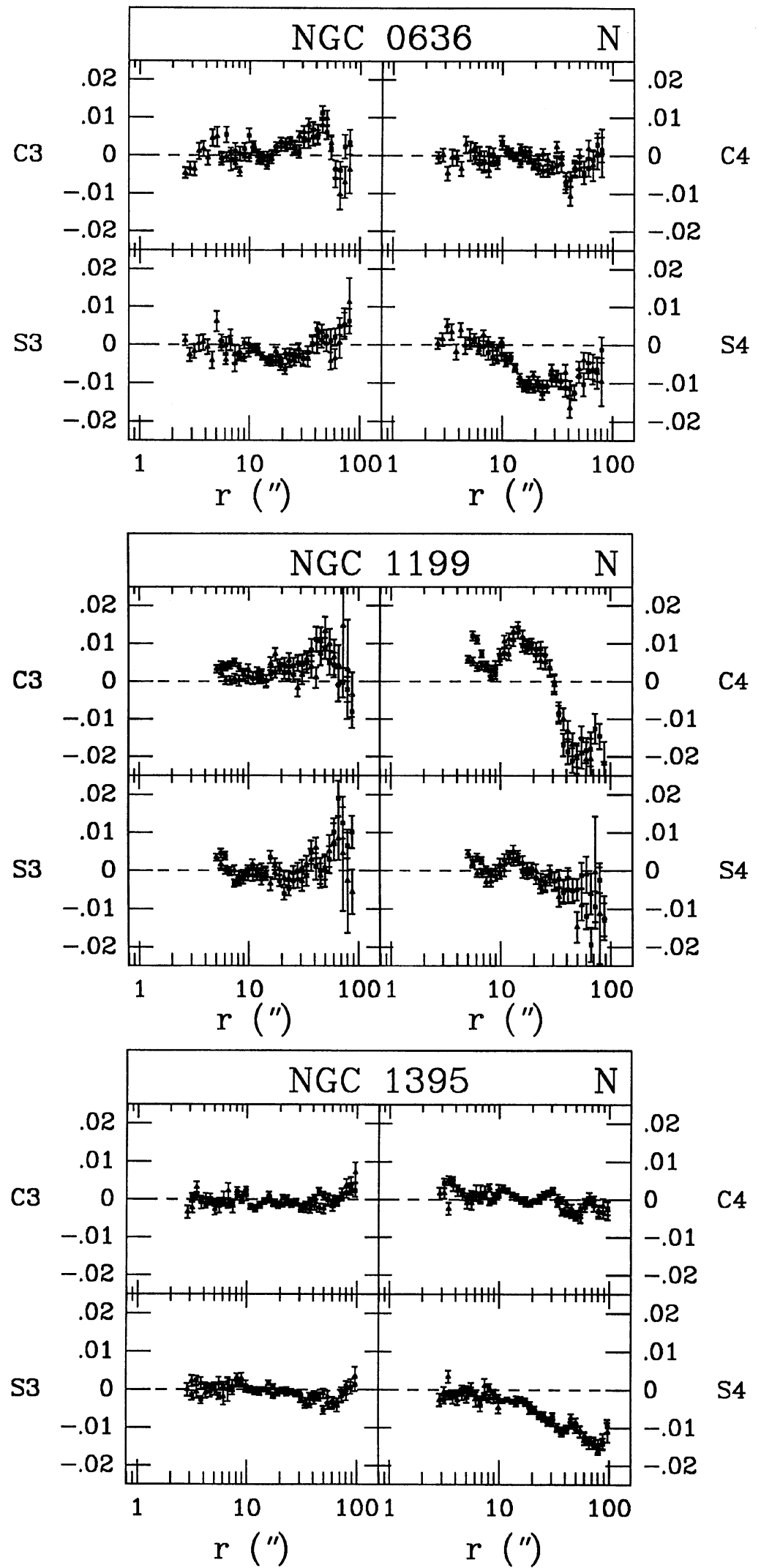

FIG. 6. High-order residuals (cos and $\sin 3 \phi$ and $4 \phi$ ) for NGC 636, NGC 1199, NGC 1395, NGC 1549 , and NGC 1700, plotted with respect to a fixed position angle (north), instead of the major axis of the galaxy. Compare these with the residuals in Fig. 9, which are given with respect to the major-axis position angle. 

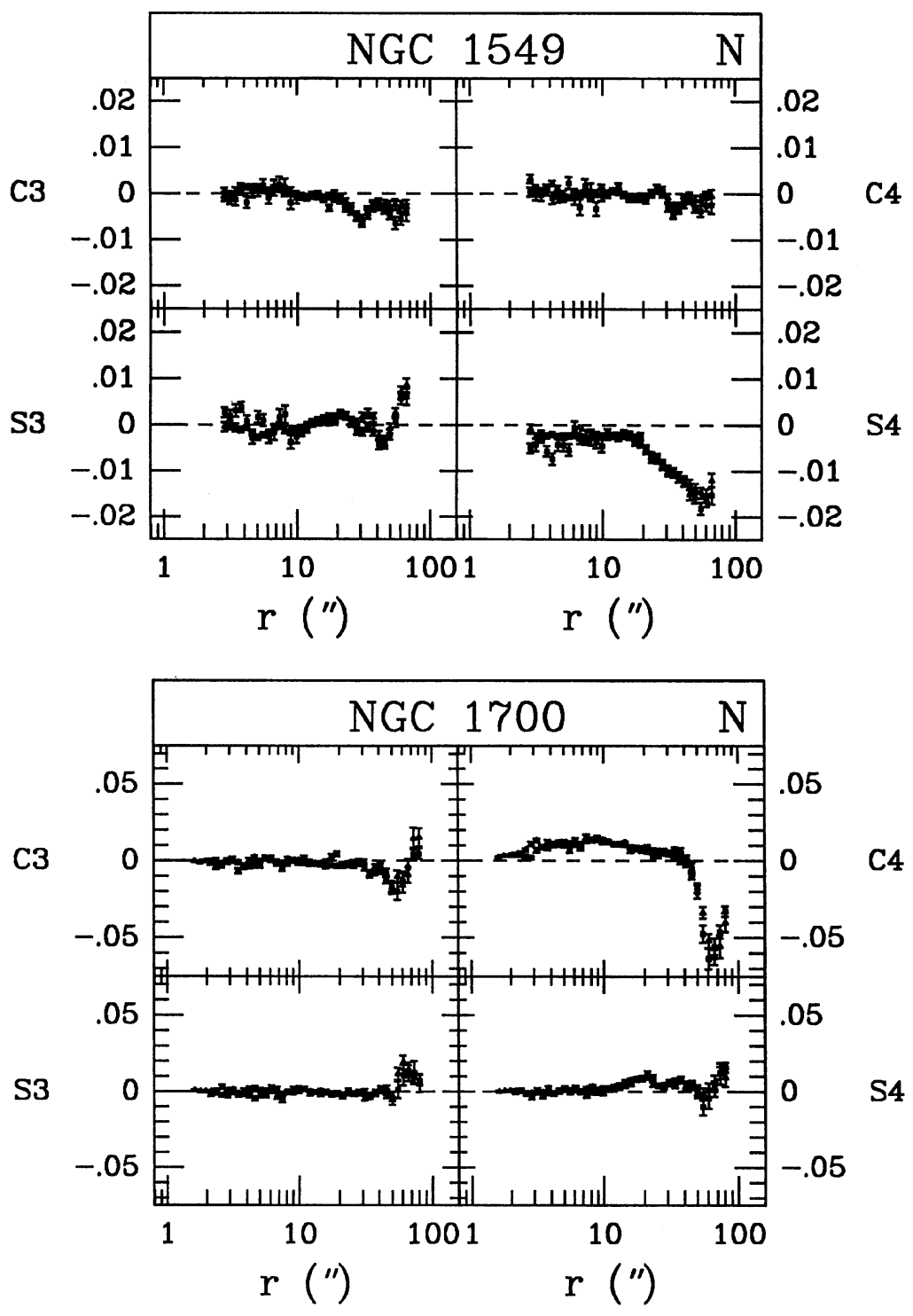

FIG. 6. (continued)

FWHM of the seeing ( $\approx 12 \sigma$ of the seeing). These results indicate that caution is required in interpreting the ellipticity and position-angle profiles, as well as the surface-brightness profiles in the inner $10^{\prime \prime}-15^{\prime \prime}$ of galaxies. The concern about the seeing notwithstanding, it is striking how accurately and reproducibly we can now determine position angles and ellipticities for even very round galaxies-in fact, for galaxies with ellipticities as small as 0.03 .

The structural profiles and the high-order residuals described by the cos and $\sin 3-4 \phi$ harmonics show considerable detail, which reproduces well in all colors and compares well with the data in the literature. In particular, some galaxies show large position-angle twists, which are naturally explained by changes in the axial ratios of triaxial galaxies. In general, triaxial galaxies with changing axial ratios will show twists in projection, but Franx (1988) has shown that there exists a large class of triaxial models that have changing axial ratios but show no twist when projected. These nontwisting models do have changing ellipticities in projection, as is commonly seen amongst ellipticals. Axisymmetric models are a small subset of this class. Thus, as has been noted before, conclusions regarding the intrinsic shape based only on the projected shape are fraught with uncertainty. In particular, on the basis of this material alone we cannot conclude that the nontwisting galaxies are oblate, even if the fraction of nontwisting ellipticals appears to be high.

The power of the high-order harmonical terms, notably the $\cos (4 \phi)$ and the $\sin (4 \phi)$ terms, to indicate the presence of weak disk- or ring-like structures was exemplified by the detection of a skew ringlike structure in NGC 1700. In general, such weak disk- or ring-like structures will result in measurable fourth-order terms, whose phase will depend on the angle between the disk or ring and the main body of the galaxy. Thus, negative $\cos (4 \phi)$ terms could indicate a skew ring or disk, and not only the usually assumed "boxiness." Careful inspection of the residuals and especially the posi- 


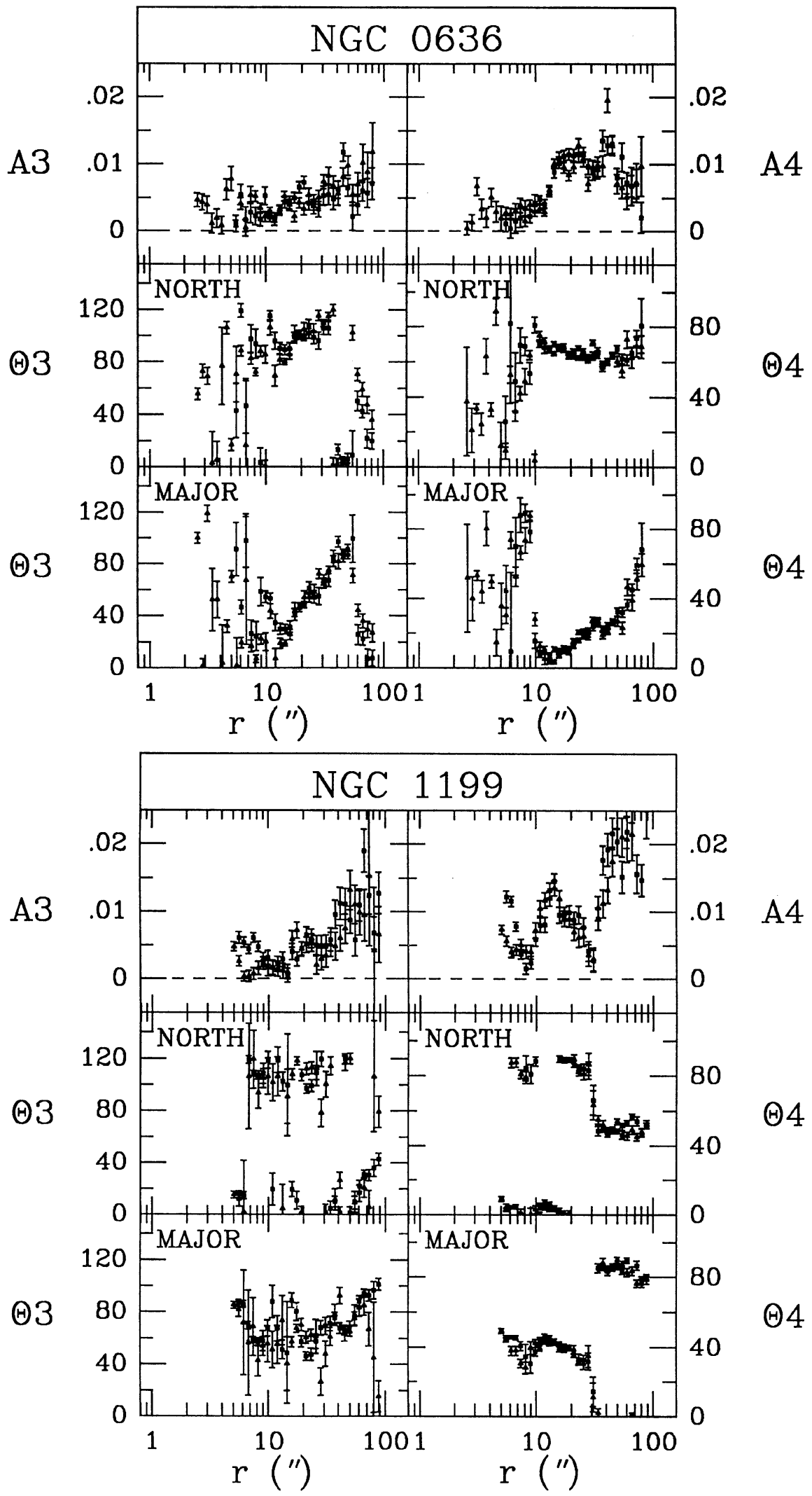

FIG. 7. Amplitudes and phases of the high-order residual terms ( $3 \phi$ and $4 \phi$ ) for NGC 636, NGC 1199, NGC 1395, NGC 1549, and NGC 1700. The phases labeled NORTH are plotted with respect to a fixed position angle (north), while the phases labeled MAJOR are plotted with respect to the major axis of the galaxy. The constant phase of some features is striking, when compared to the large changes in major-axis position angle. 

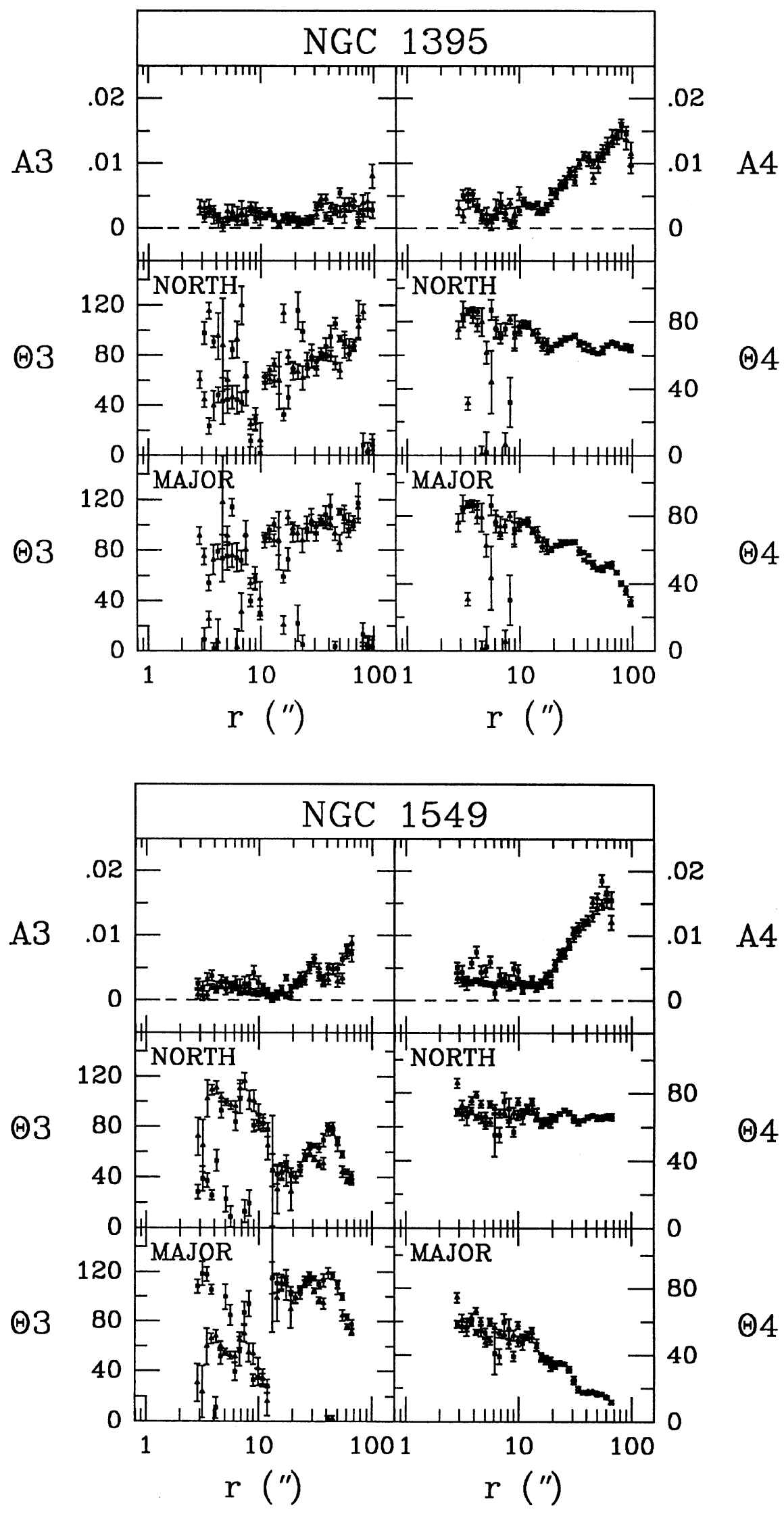

FIG. 7. (continued) 


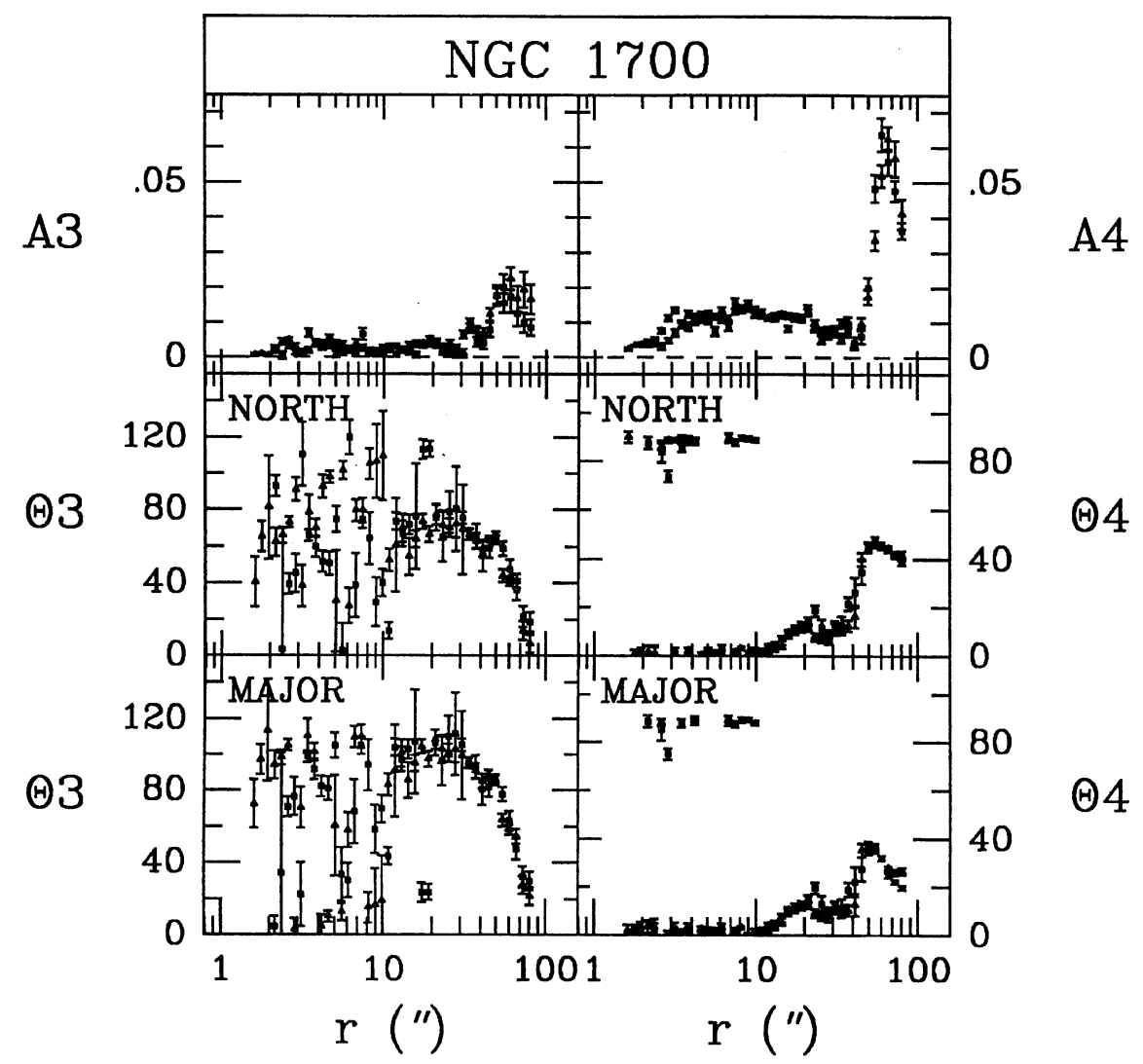

Fig. 7. (continued)

tion-angle profile might allow one to distinguish the presence of a ring/disk or the more symmetrical boxiness. Residuals beyond fourth order could be useful here as well, given adequate $\mathbf{S} / \mathbf{N}$. In this latter case of boxiness, it has been suggested that the negative $\cos (4 \phi)$ terms are due to a relatively high population of thin tube orbits or boxes in galaxies, caused possibly by mergers or acquisitions of low-luminosity galaxies (see, e.g., Binney and Petrou 1985; Whit-

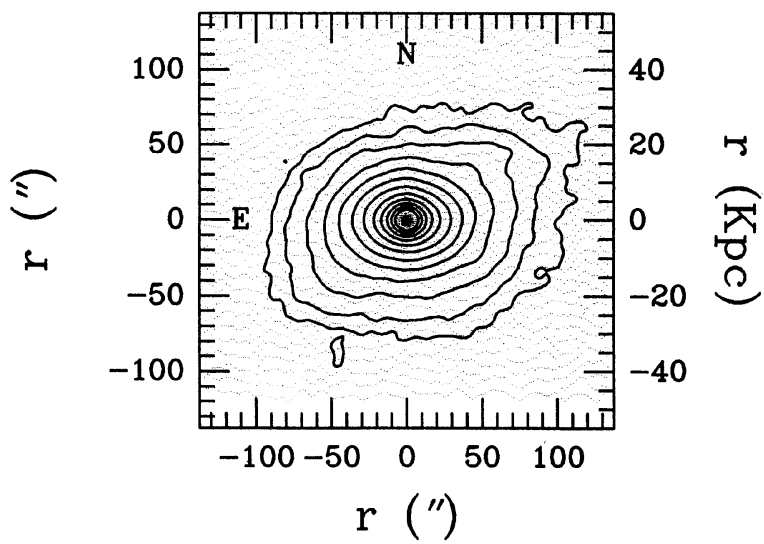

FIG. 8. Contours of constant $B$ surface brightness of NGC 1700. The contours are drawn at intervals of $0.5 \mathrm{mag}$. The outermost contour corresponds to a surface brightness of $25 \mathrm{mag} \operatorname{arcsec}^{-2}$. The amplitude of the distortion at $50^{\prime \prime}-70^{\prime \prime}$ is apparent. more and Bell 1988; Statler 1988). It is not clear whether the low-level harmonical terms $(\leqslant 0.5 \%)$ that we find for many galaxies have any special meaning. They may be related to the subcomponents that are apparent in the kinematics of some ellipticals (e.g., IC 1459-Franx and Illingworth 1988).

Color gradients appear to be almost universal in our sample, with a mean $U-R$ gradient of -0.23 mag per decade in radius, and a mean $B-R$ gradient of -0.07 . Some uncertainty still exists because of the uncertainty in the background subtraction. In only one galaxy do we find a very clear signature of absorption by dust, NGC 1199, while for two other galaxies (IC 1459, NGC 7507) we find a slightly asymmetric color image. However, the high detection rate of $100 \mu \mathrm{m}$ emission from ellipticals with IR $A S$ data (Jura et al. 1987 ) suggests that a sizeable fraction may have dust that is not found in the surface photometry. On the basis of this photometric data alone, we cannot exclude the possibility that the color gradients could in part be caused by dust absorption; one would require that the dust column density decrease slowly with radius. Such a nearly uniform distribution of dust across the galaxy would be highly unlikely. Hence the color gradients are thought to be produced by metallicity and/or age gradients within the galaxy. It is well known that the strengths of metal absorption lines in ellipticals show radial gradients (Faber 1977; Gorgas and Efstathiou 1987; Davies and Sadler 1987), with the stronger metal absorption lines being found in the centers. It is likely that such gradients reflect metallicity and/or metallicity-driven 
population changes. With the color-metallicity relation from Arimoto and Yoshii (1987), we derive a mean metallicity gradient from our mean $U-R$ gradient of $\Delta \log Z$ / $\Delta \log r=-0.28$. The change in metallicity is about a factor of 2 per decade in radius, in the sense that the outer parts have a lower metallicity. For a more extensive discussion of the interpretation of these color gradients, we refer to a forthcoming paper (Franx and Illingworth 1989).

It is a pleasure to thank Jeanette Barnes and Lindsey Davis, who helped us with the data reduction in the initial phase of the project. Discussions with Reynier Peletier, Roger Davies, and Rudolf le Poole are acknowledged. Reynier Peletier and Tim de Zeeuw gave comments on an earlier draft of the paper. The Leids Sterrewacht Fonds and the Leiden Kerkhoven-Bosscha Fonds supported M.F. during part of this work. M.F. wishes to thank the Kitt Peak National Observatory and, particularly, the Space Telescope Science Institute for their hospitality.

\section{APPENDIX A: SECOND-ORDER EXPANSION OF SEEING EFFECTS}

The effects of the seeing on the observed profiles of intensity, ellipticity, and position angle are derived for the case of a noncircularly symmetric PSF. It is assumed that the seeing effects are small. The seeing function $f$ or PSF is of the form [see also Eq. (5)]

$$
f(x, y)=\frac{1}{s^{2}} g\left(\frac{x^{\prime 2} / p+p y^{\prime 2}}{s^{2}}\right),
$$

with $p \geqslant 1$ and

$$
\left(\begin{array}{l}
x^{\prime} \\
y^{\prime}
\end{array}\right)=\left(\begin{array}{cc}
\cos \psi & \sin \psi \\
-\sin \psi & \cos \psi
\end{array}\right)\left(\begin{array}{l}
x \\
y
\end{array}\right) \text {. }
$$

The parameter $p$ is the elongation of the PSF, and is related to the ellipticity $\delta$ of the PSF by $\delta=1-1 / p$. The angle $\psi$ is the position angle of the longest axis of the PSF, measured anticlockwise from the $x$ axis. The moments of the seeing function $F_{i, j}$ are defined by

$$
F_{i, j}=\int_{-\infty}^{\infty} \int_{-\infty}^{\infty} x^{i} y^{j} f(x, y) d x d y .
$$

We require that $f$ be normalized, hence $F_{0,0}=1$. In the following, we also require that $F_{0,1}=F_{1,0}=0$, which can be achieved by a shift of the function with respect to the origin. The moments $F_{i, j}$ are related to the moments $G_{i, j}$ of $g$ by

$$
F_{i, j}=s^{i+j} G_{i, j} \text {. }
$$

Now assume that the surface brightness $I$ of the galaxy around an arbitrary point $\left(x_{0}, y_{0}\right)$ can be expanded as a power series in $\delta x, \delta y$, with $\delta x=x-x_{0}, \delta y=y-y_{0}$,

$$
I=\sum_{i=0}^{\infty} \sum_{j=0}^{\infty} I_{i, j} \delta x^{i} \delta y^{j}
$$

By taking the $i$ th derivative with respect to $\delta x$ and the $j$ th derivative with respect to $\delta y$, we derive for the terms $I_{i, j}$

$$
I_{i, j}=\left.\frac{1}{i ! j !} \frac{\partial^{i}}{\partial x^{i}} \frac{\partial^{j}}{\partial y^{j}} I(x, y)\right|_{x=x_{0}, y=y_{0}} .
$$

After convolution with a PSF $f(x, y)$, the observed surface brightness $I^{\prime}\left(x_{0}, y_{0}\right)$ is

$$
\begin{aligned}
I^{\prime} & \left(x_{0}, y_{0}\right) \\
& =\int I\left(\mathbf{x}_{0}-\mathbf{x}\right) f(\mathbf{x}) d \mathbf{x} \\
& =\int_{-\infty}^{\infty} \int_{-\infty}^{\infty} \sum_{i=0}^{\infty} \sum_{j=0}^{\infty}(-1)^{i+j} I_{i, j} x^{i} y j f(x, y) d x d y \\
& =\sum_{i=0}^{\infty} \sum_{j=0}^{\infty}(-1)^{i+j} I_{i, j} F_{i, j} .
\end{aligned}
$$

Thus the observed surface brightness $I^{\prime}$ can be expressed as

$$
\begin{aligned}
I^{\prime} & =\sum_{i=0}^{\infty} \sum_{j=0}^{\infty} I_{i, j} G_{i, j} s^{i+j}(-1)^{i+j} \\
& =I_{0,0}+\left(I_{2,0} G_{2,0}+I_{1,1} G_{1,1}+I_{0,2} G_{0,2}\right) s^{2}+O\left(s^{3}\right) .
\end{aligned}
$$

The error in the observed surface brightness $\Delta I \equiv I^{\prime}-I$ is to second order in $S$

$$
\Delta I=\left(I_{2,0} G_{2,0}+I_{1,1} G_{1,1}+I_{0,2} G_{0,2}\right) s^{2} .
$$

Hence, to second order the error $\Delta I$ depends only on the second-order moment of the PSF. We proceed to derive the effect of the error $\Delta I$ on the intensity, ellipticity, and position angle measured by the ellipse-fitting program.

The moments $F_{i, j}$ of the PSF can be calculated as a function of the elongation $p$ and angle $\psi$ of the PSF by evaluation of

$$
\begin{aligned}
F_{i, j}= & \int_{-\infty}^{\infty} \int_{-\infty}^{\infty} x^{i} y^{j} f(x, y) d x d y \\
= & \int_{-\infty}^{\infty} \int_{-\infty}^{\infty} \frac{1}{s^{2}}\left(x^{\prime} \cos \psi-y^{\prime} \sin \psi\right)^{i} \\
& \times\left(x^{\prime} \sin \psi+y^{\prime} \cos \psi\right)^{j} g\left(\frac{x^{\prime 2}}{s^{2} p}+\frac{p y^{\prime 2}}{s^{2}}\right) d x^{\prime} d y^{\prime}
\end{aligned}
$$

We obtain for the second-order moments

$$
\begin{aligned}
& F_{2,0}=\frac{1}{2}\left(p \cos ^{2} \psi+\frac{\sin ^{2} \psi}{p}\right) s^{2} G_{2}, \\
& F_{0,2}=\frac{1}{2}\left(p \sin ^{2} \psi+\frac{\cos ^{2} \psi}{p}\right) s^{2} G_{2}, \\
& F_{1,1}=\frac{1}{2} \sin \psi \cos \psi\left(p-\frac{1}{p}\right) s^{2} G_{2},
\end{aligned}
$$

where

$$
G_{2}=\int r^{2} g\left(r^{2}\right) d \mathbf{r},
$$

which is independent of the elongation $p$ and angle $\psi$. The second-order moment $F_{2}$ of the PSF is defined to be

$$
F_{2}=s^{2} G_{2}=\int r^{2} F(\mathbf{r}) d \mathbf{r} .
$$

We assume that the surface brightness of the galaxy is constant on similar concentric ellipses, and that the $x$ axis and the $y$ axis are symmetry axes. We write

$$
I(x, y)=J\left(m^{2}\right),
$$

with

$$
m^{2}=\frac{x^{2}}{q}+q y^{2}
$$

Hence the ellipticity of the galaxy is equal to $\epsilon=1-1 / q$. Note that $I$ denotes the intensity of the galaxy at an arbitrary point, while $J$ denotes the one-dimensional profile of the gal- 
axy. The second-order expansion coefficients at an arbitrary point are now

$$
\begin{aligned}
& I_{2,0}=\frac{1}{2} \frac{\partial^{2}}{\partial x^{2}} J\left(\frac{x^{2}}{q}+q y^{2}\right)=\frac{1}{q} J^{\prime}+\frac{2 x^{2}}{q^{2}} J^{\prime \prime}, \\
& I_{1,1}=\frac{\partial}{\partial x} \frac{\partial}{\partial y} J\left(\frac{x^{2}}{q}+q y^{2}\right)=4 x y J^{\prime \prime}, \\
& I_{0,2}=\frac{1}{2} \frac{\partial^{2}}{\partial y^{2}} J\left(\frac{x^{2}}{q}+q y^{2}\right)=q J^{\prime}+2 y^{2} q^{2} J^{\prime \prime},
\end{aligned}
$$

where $J^{\prime}$ and $J^{\prime \prime}$ are the first and second derivatives of $J\left(\mathrm{~m}^{2}\right)$ with respect to $m^{2}$. The change in surface brightness $\Delta I$ is now for small $s$

$$
\begin{aligned}
\Delta I= & \frac{1}{2}\left[\frac{1}{q}\left(p \cos ^{2} \psi+\frac{\sin ^{2} \psi}{p}\right)\right. \\
& \left.+q\left(p \sin ^{2} \psi+\frac{\cos ^{2} \psi}{p}\right)\right] J^{\prime} s^{2} G_{2} \\
& +\left[\frac{x^{2}}{q^{2}}\left(p \cos ^{2} \psi+\frac{\sin ^{2} \psi}{p}\right)\right. \\
& +2 x y \sin \psi \cos \psi\left(p-\frac{1}{p}\right) \\
& \left.+q^{2} y^{2}\left(p \sin ^{2} \psi+\frac{\cos ^{2} \psi}{p}\right)\right] J^{\prime \prime} s^{2} G_{2} .
\end{aligned}
$$

The change in measured surface brightness, ellipticity, and position angle can be derived from the harmonical terms of $\Delta I$ along the ellipse $I=$ constant. Along the ellipse, $J^{\prime}$ and $J^{\prime \prime}$ are constant, which simplifies the calculations considerably. The changes in ellipticity $\Delta \epsilon$ and in position angle $\Delta \theta$ are related to the harmonical terms by (e.g., Jedrzejewski 1987)

$$
\begin{aligned}
& \Delta \epsilon=\frac{-2 C_{2}(1-\epsilon)}{2 m^{2} J^{\prime}}, \\
& \Delta \Theta=\frac{2 S_{2}(1-\epsilon)}{2 m^{2} J^{\prime}\left[(1-\epsilon)^{2}-1\right]} .
\end{aligned}
$$

where the factors $C_{2}$ and $S_{2}$ are the amplitudes of the $\cos (2 \phi)$ and $\sin (2 \phi)$ harmonical terms. The change in measured surface brightness, ellipticity, and position angle can be calculated in a straightforward way:

$$
\begin{aligned}
\Delta J= & \frac{1}{2}\left[\frac{1}{q}\left(p \cos ^{2} \psi+\frac{\sin ^{2} \psi}{p}\right)\right. \\
& \left.+q\left(p \sin ^{2} \psi+\frac{\cos ^{2} \psi}{p}\right)\right]\left(J^{\prime}+m^{2} J^{\prime \prime}\right) s^{2} G_{2}, \\
\Delta \epsilon= & -\frac{1}{2}\left[\frac{1}{q}\left(p \cos ^{2} \psi+\frac{\sin ^{2} \psi}{p}\right)\right. \\
& \left.-q\left(p \sin ^{2} \psi+\frac{\cos ^{2} \psi}{p}\right)\right] \frac{1}{q} \frac{J^{\prime \prime}}{J^{\prime}} s^{2} G_{2}, \\
\Delta \Theta= & -\sin \psi \cos \psi \frac{(p-1 / p)}{(q-1 / q)} \frac{J^{\prime \prime}}{J^{\prime}} s^{2} G_{2} .
\end{aligned}
$$

For small ellipticity $\epsilon$ of the galaxy and $\delta$ of the PSF, the errors are to first order in $\epsilon, \delta$

$$
\begin{aligned}
& \Delta J=\left(J^{\prime}+m^{2} J^{\prime \prime}\right) s^{2} G_{2}, \\
& \Delta \epsilon=(\epsilon-\delta \cos 2 \psi) \frac{J^{\prime \prime}}{J^{\prime}} s^{2} G_{2}, \\
& \Delta \Theta=-\sin \psi \cos \psi \frac{\delta}{\epsilon} \frac{J^{\prime \prime}}{J^{\prime}} s^{2} G_{2} .
\end{aligned}
$$

These formulas are used and discussed in Sec. III.

\section{APPENDIX B: DATA ON INDIVIDUAL GALAXIES}

The data are listed in Tables IX(a)-IX (q). The surface brightness and ellipticity are given over the full radial range. The errors for the ellipticity are not given at radii where the seeing effects are strong. The seeing effects are included in the errors for every third datum for the surface brightness, color, surface brightness, ellipticity, and position angle. Lower limits are given for the errors in surface brightness, color, ellipticity, position angle, and residual harmonical terms of $0.01,0.01,0.01,1^{\circ}$, and $0.1 \%$, respectively, whenever the estimated errors fall below these values. The systematic errors are thought to be of this order or larger.

Figures 9(a)-9(q) give the profiles of $R$ intensity, $B-R$ and $U-R$ colors, ellipticity, position angle, and cosine and sine $3 \phi$ and $4 \phi$ terms. The apparent shape profiles for $B$ and $R$ are plotted, except for NGC 7619, where the $B$ data were corrupted by a very high sky level. Error bars give the formal errors from the fit for the apparent-shape profiles. The larger error bars on every third point reflect the additional uncertainty due to the seeing for the ellipticity, position angle, $R$ surface brightness, and the colors. The error bars for the intensity and colors also include the estimated systematic errors due to possible errors in the background determination. Comments on the individual galaxies follow:

NGC 636. The position angle changes from $70^{\circ}$ to $10^{\circ}$. The isophotes deviate from ellipses by $1 \%$. Significant third- and fourth-order residuals were found. The fourth-order structure has constant phase, even though the major-axis position angle changes by $60^{\circ}$.

NGC 1199. This galaxy has a dust lane near the center, found before by Ebneter and Balick (1985) and Ebneter, Djorgovski, and Davis (1988). The negative $\cos (4 \phi)$ terms near the center are probably related to this dust lane. The galaxy has "disklike" distortions on the order of $2 \%$ in the outer parts. We also measure significant third-order residuals on a level of $1 \%$ and higher in the outer regions. The position angle changes less than $10^{\circ}$.

NGC 1379. A very round galaxy $(\epsilon<0.05)$ in Fornax. The isophotes do not show any significant deviation from ellipses, which is rare in our sample. The position angle changes by $20^{\circ}$.

NGC 1395. This galaxy is very regular at radii smaller than $20^{\prime \prime}$, but shows a twist outside that range, and deviations from ellipses on the order of $1 \%$ at larger radii. The fourth-order structure shows little phase shift. Detected by IRAS at $100 \mu \mathrm{m}$.

NGC 1399. The central galaxy in Fornax I. The shape is very regular, except for an isophote twist starting at $25^{\prime \prime}$ and increasing outwards. This galaxy may continue to twist at larger radii. Its core is probably resolved. The luminosity profile is very shallow at large radii (see also Killeen and Bicknell 1988). We measure very low distortions only at 30 ", which may be due to a bright star. Detected at $100 \mu \mathrm{m}$ and at $5 \mathrm{GHz}$.

NGC 1404. A fairly regular galaxy, with deviations from ellipses up to $0.5 \%$, and a position-angle twist of $10^{\circ}$. The ellipticity differences in $B$ and $R$ near the center are due to differences in the seeing. Detected by IRAS.

NGC 1407. The largest galaxy in our sample. Note the small ellipticity $(0.05)$ and the constant position angle. The isophotes deviate from ellipses on a low level (up to $0.5 \%$ ). It is the only galaxy for which we may have found a positive 

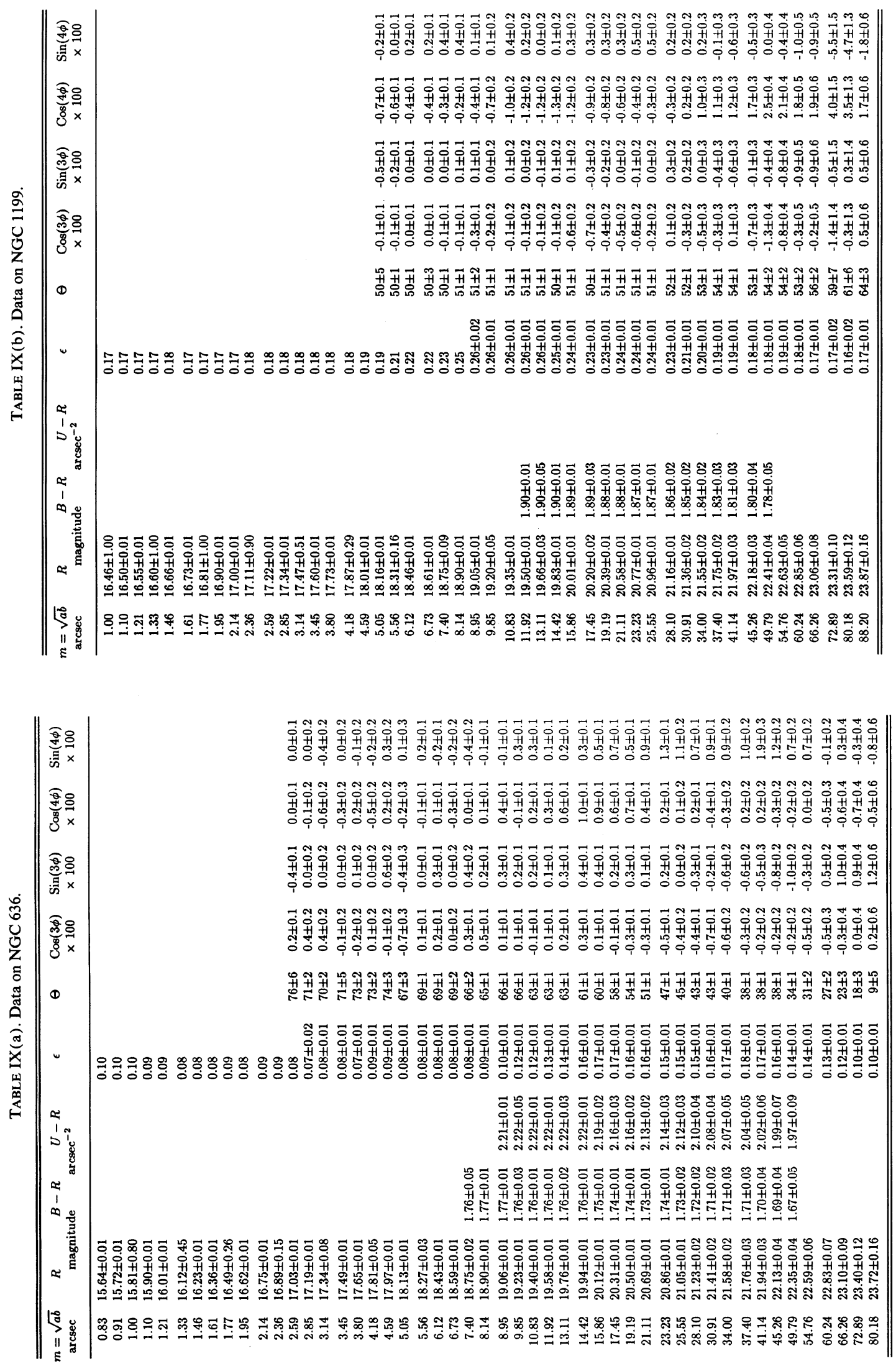

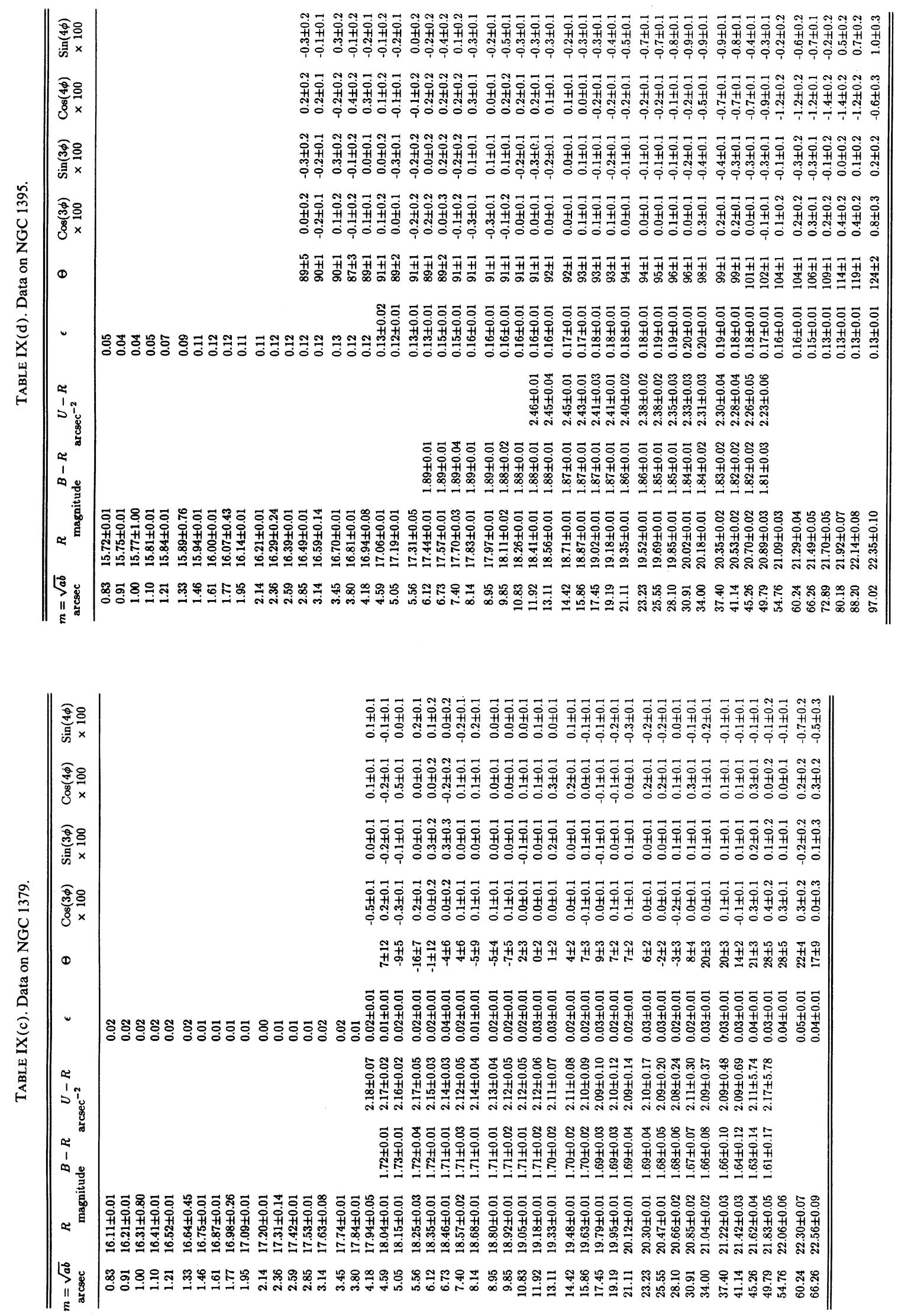


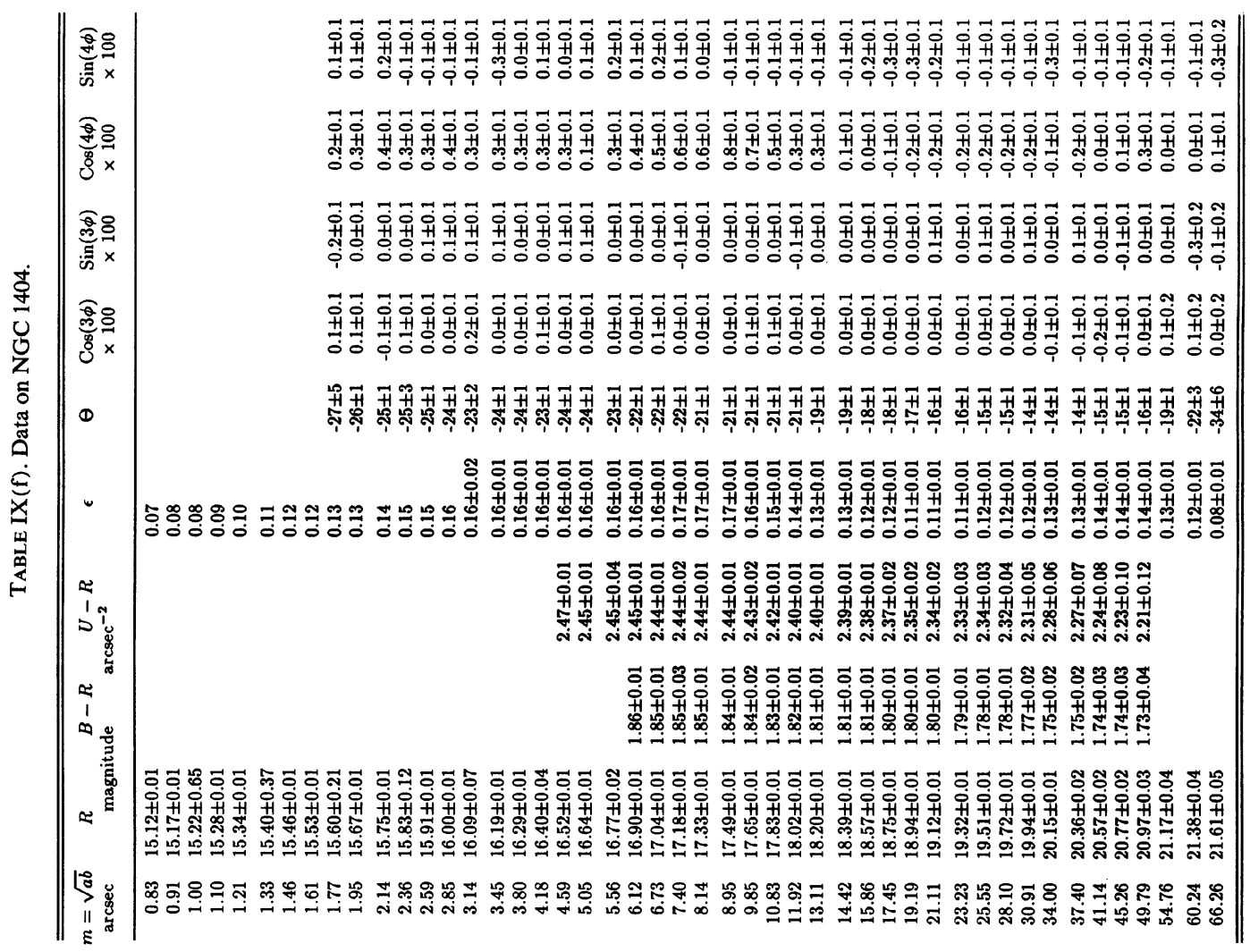

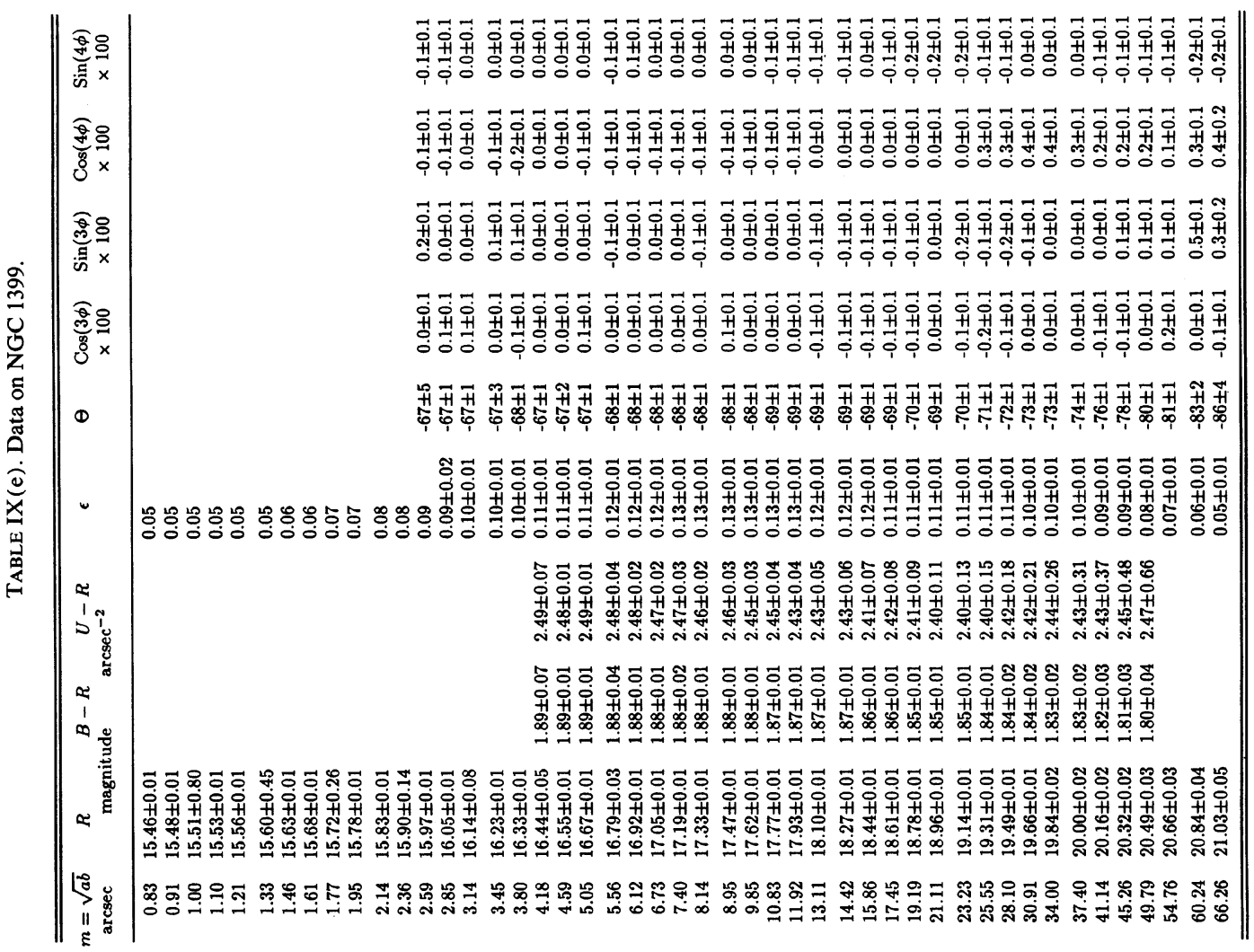



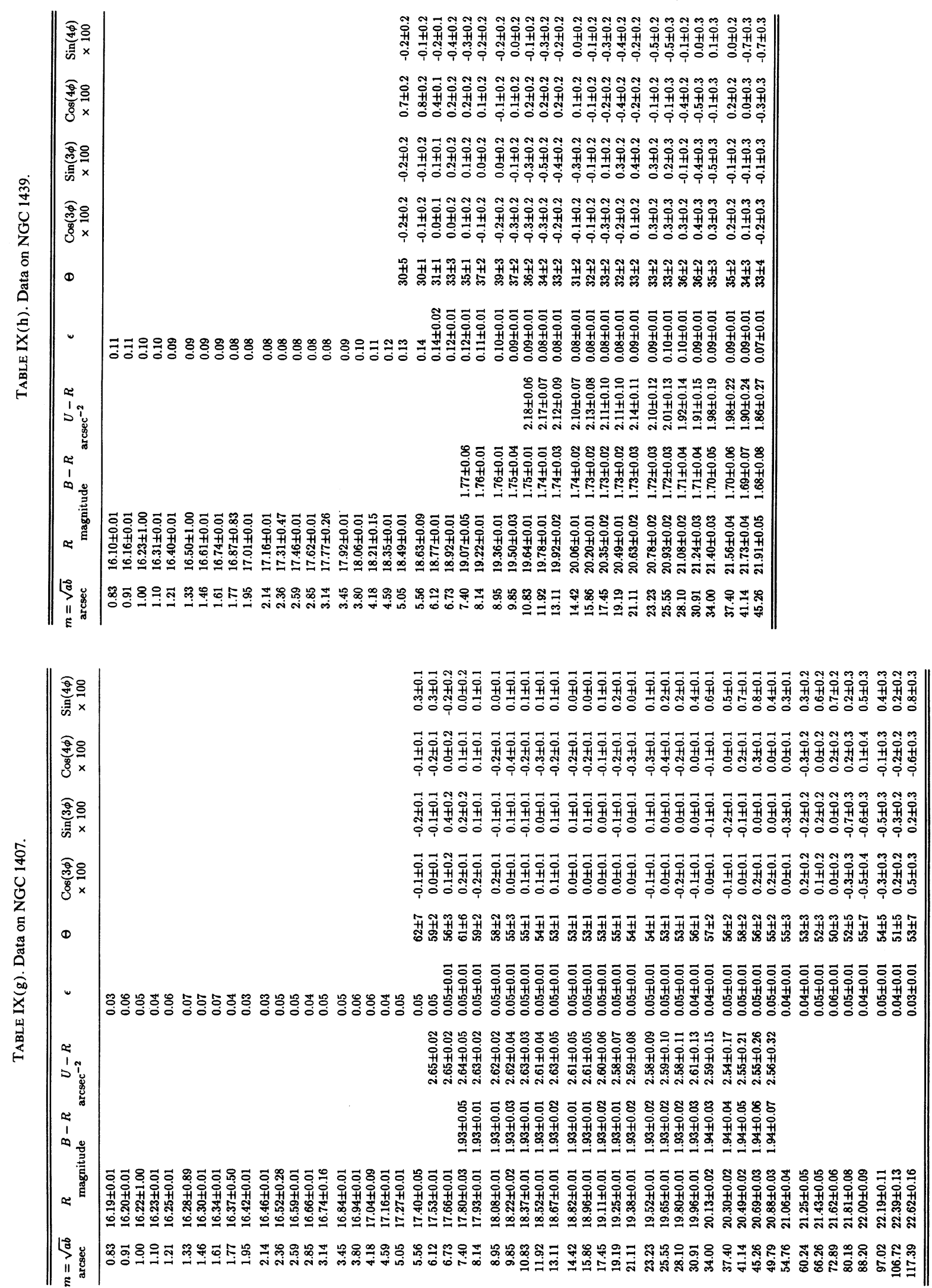

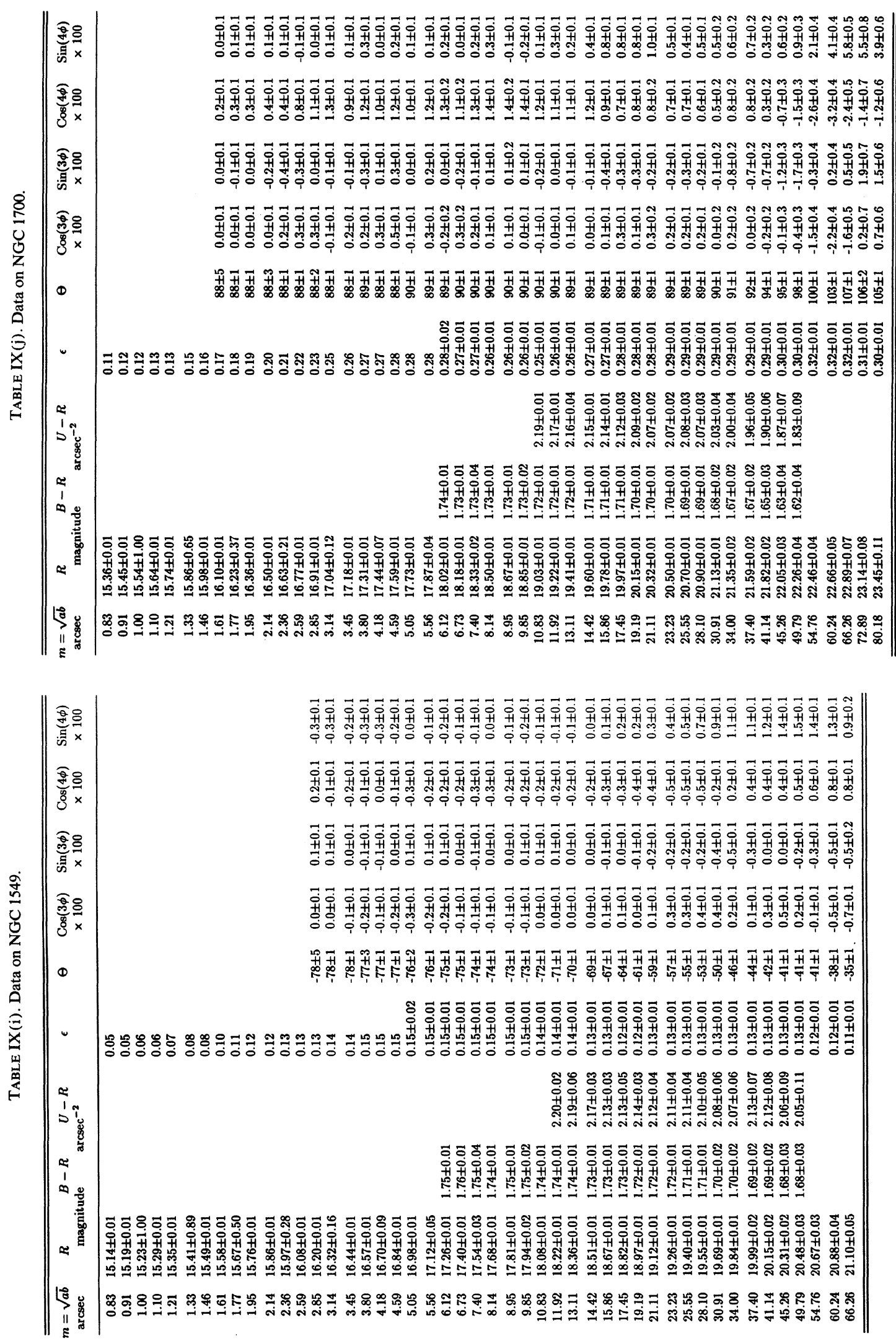

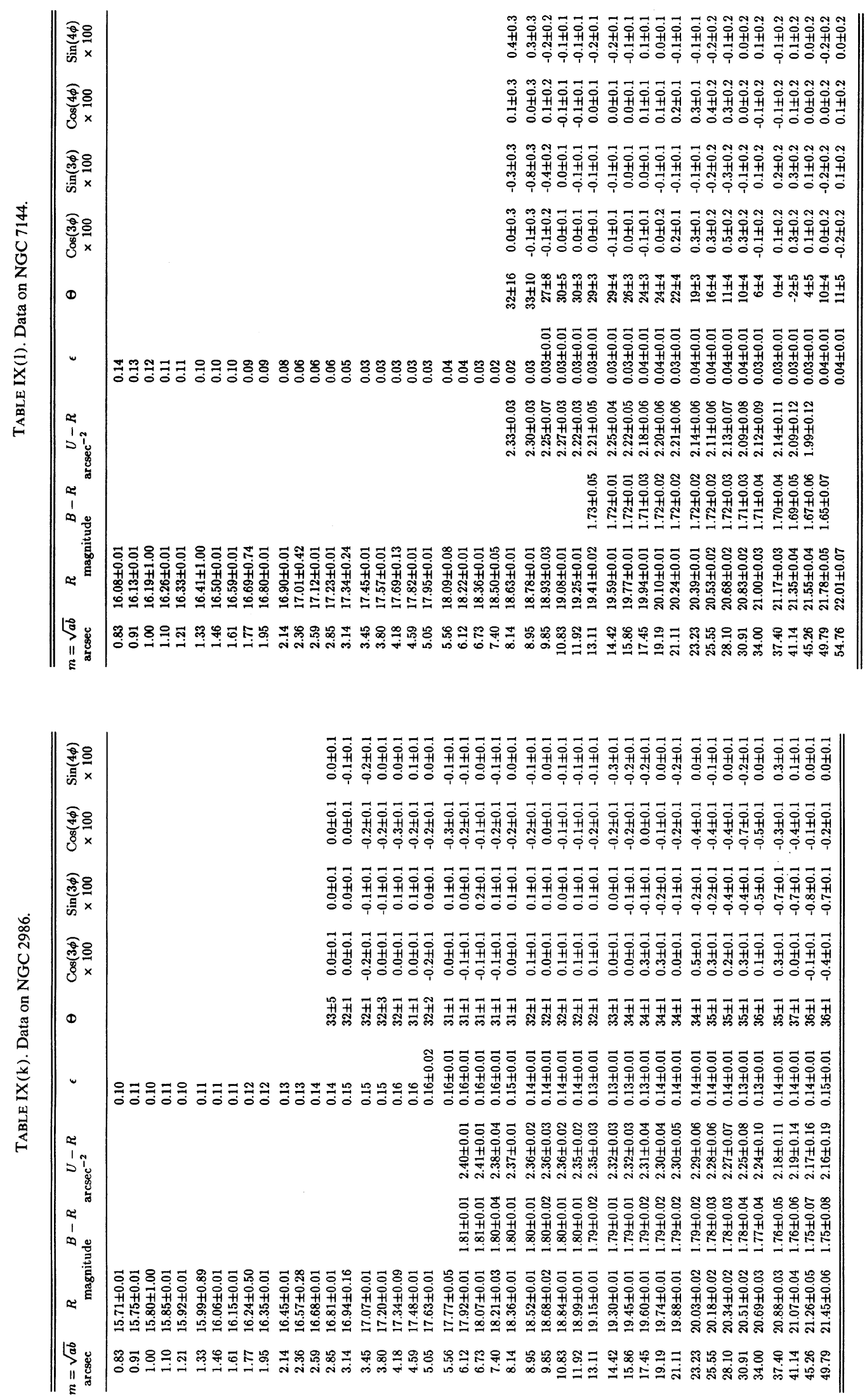

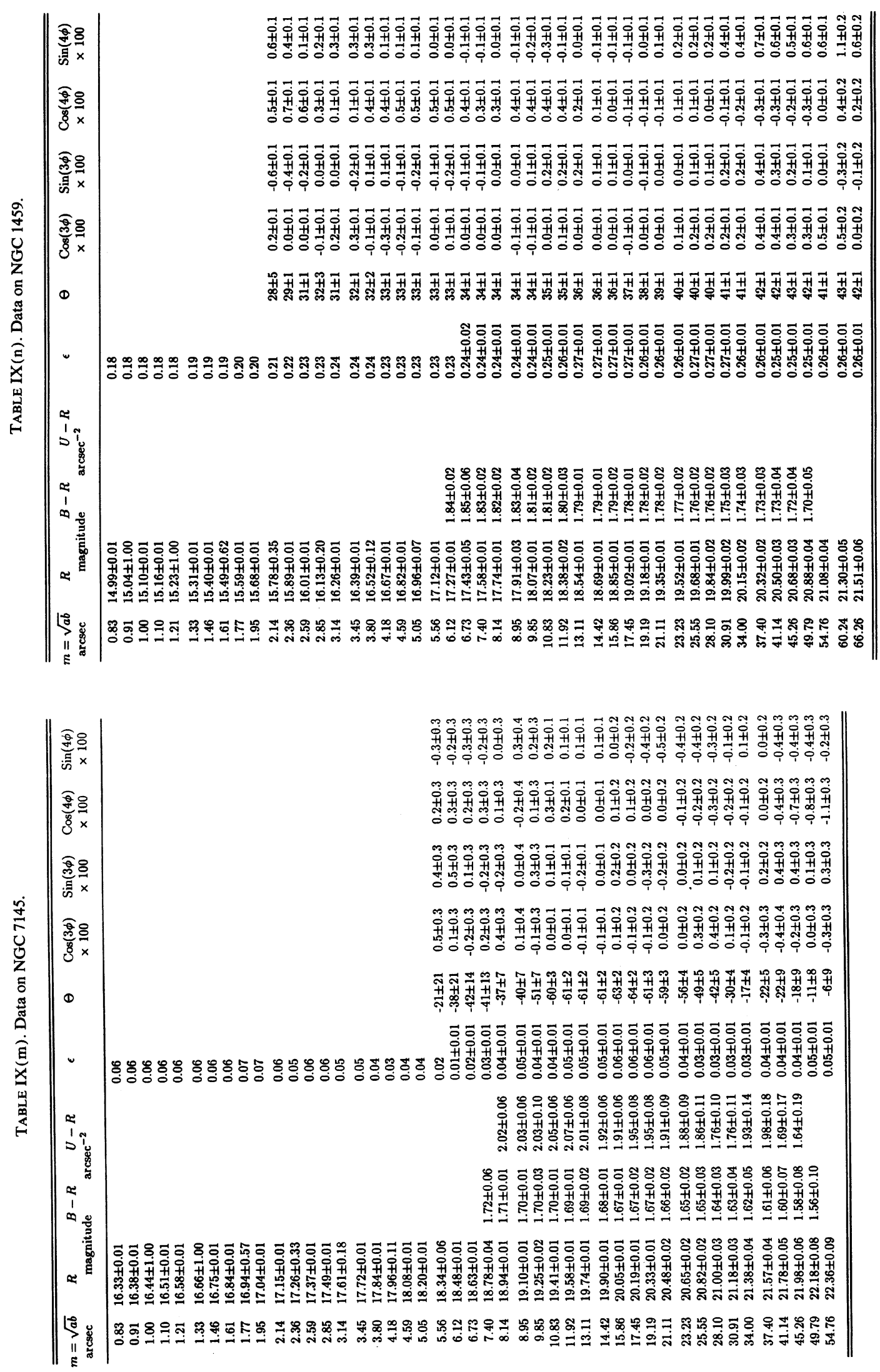

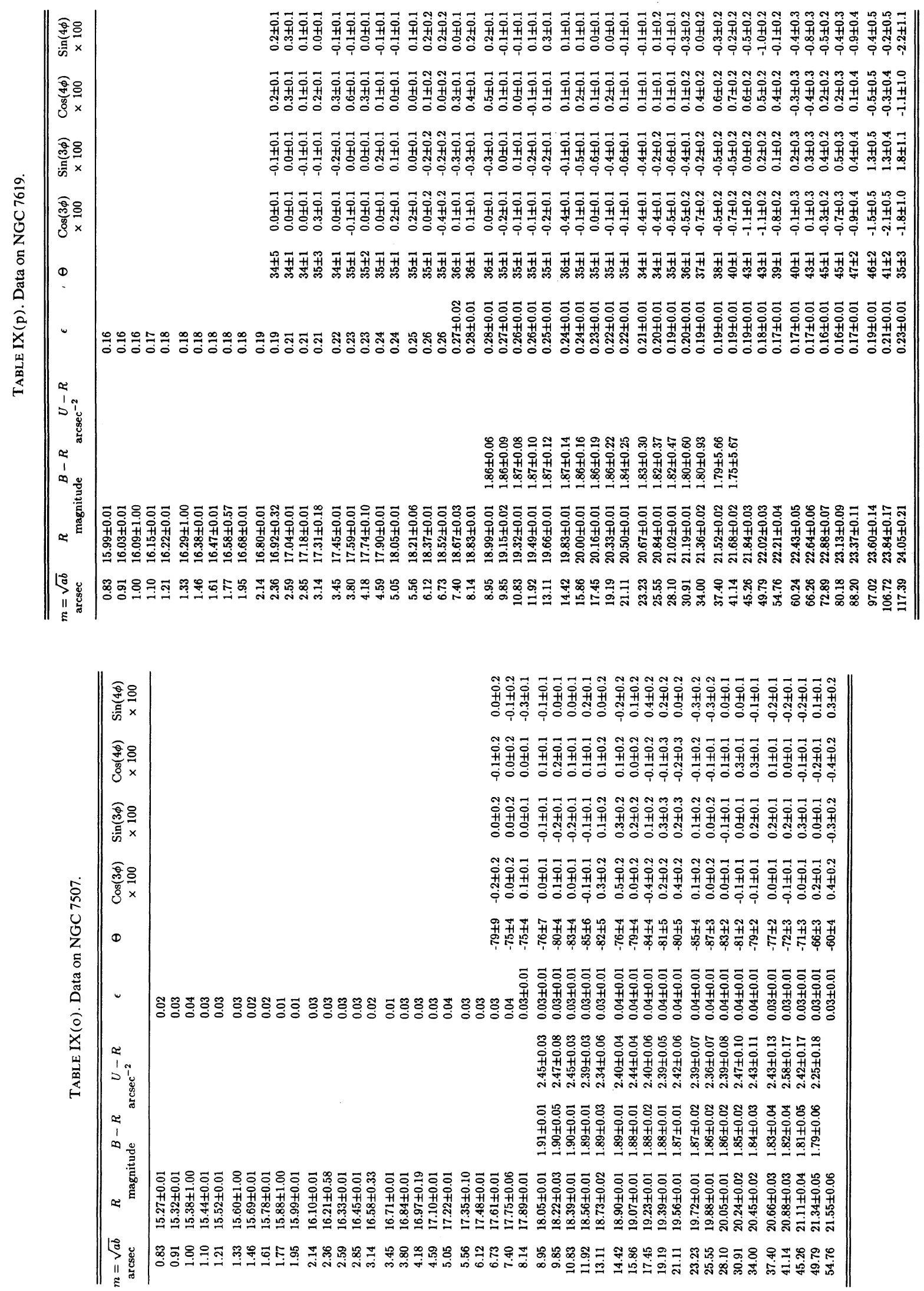


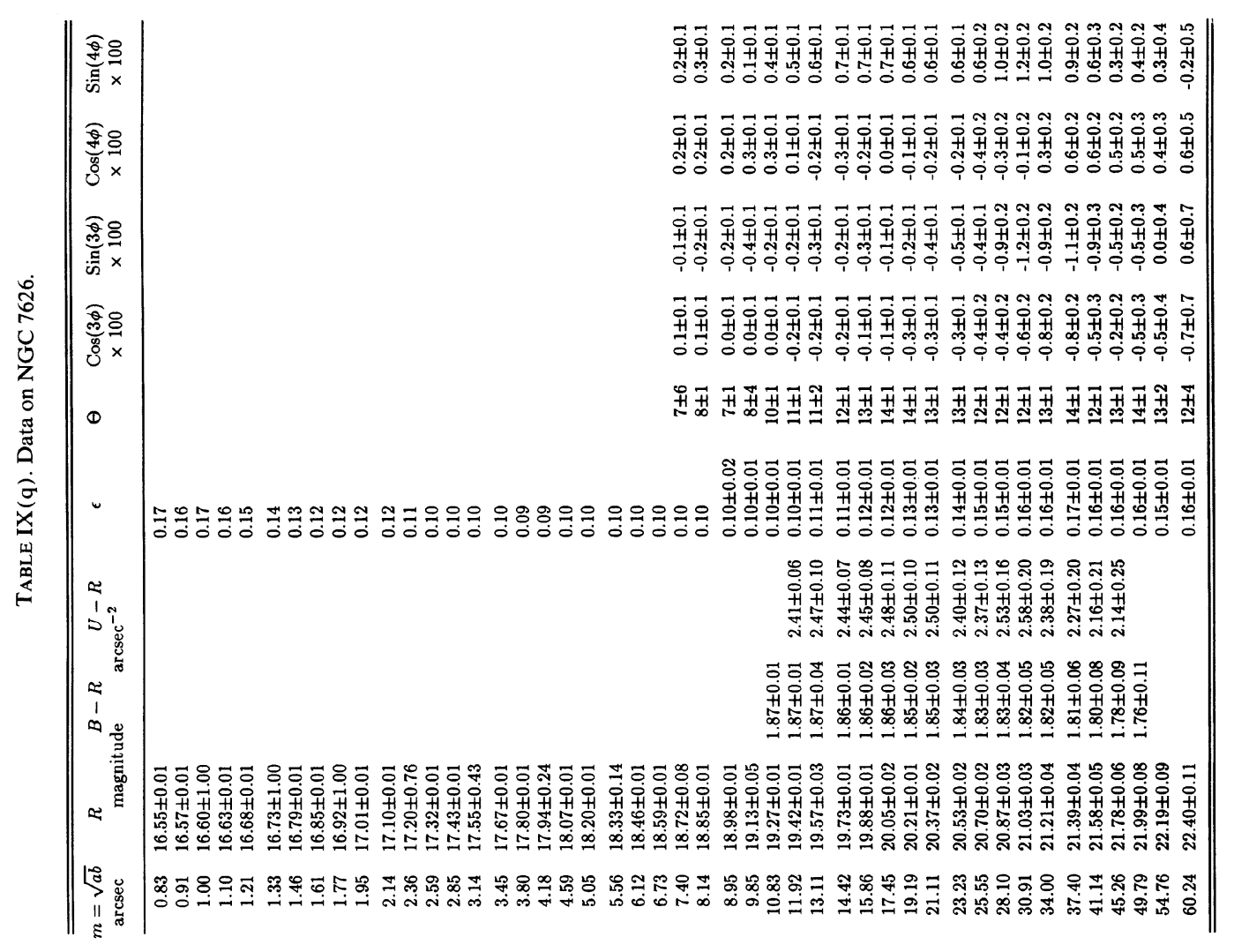



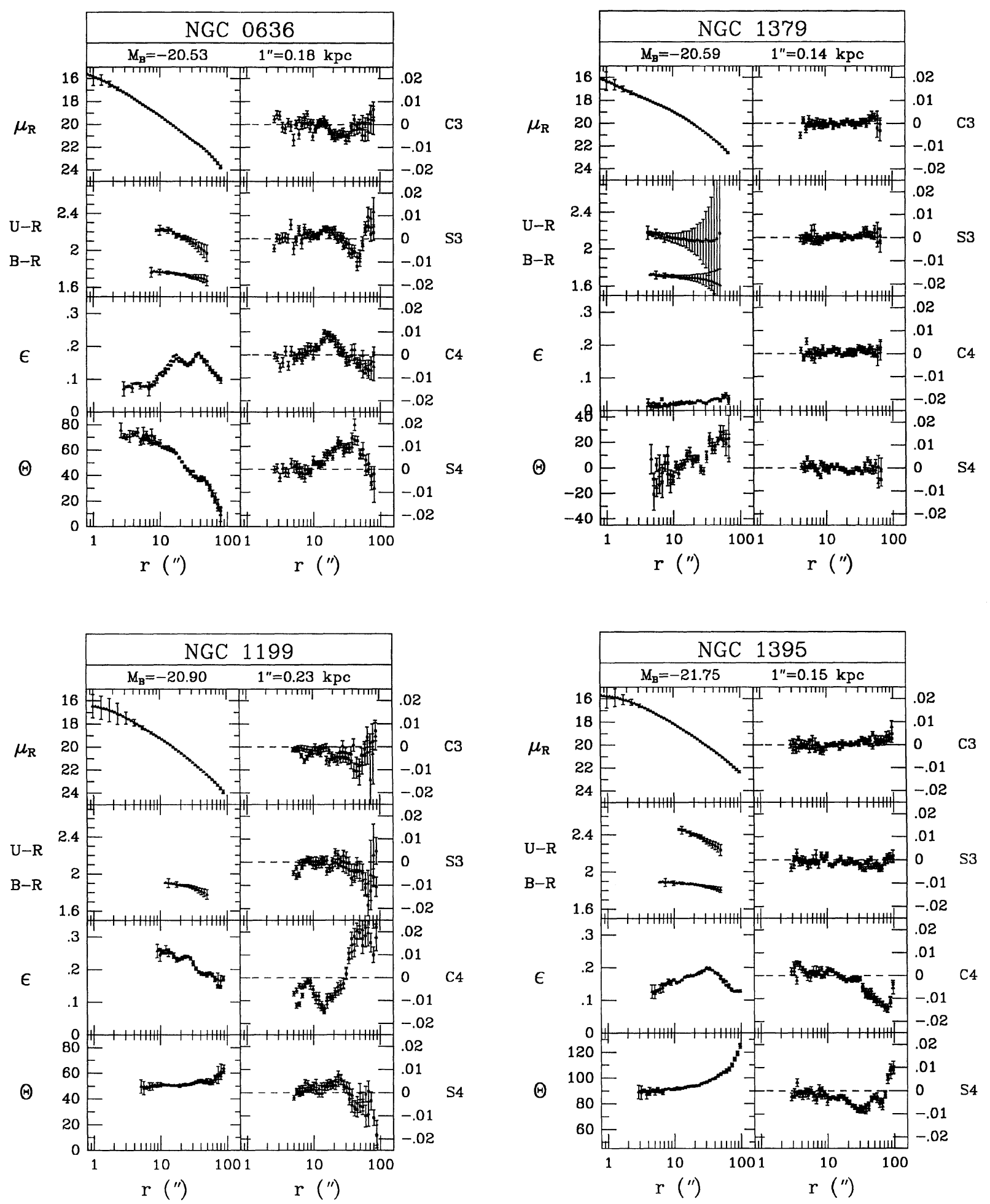

Fig. 9. Profiles of $R$ surface brightness $\left(\mu_{R}\right)$, colors ( $U-R$ and $B-R$ ), ellipticity $(\epsilon)$, position angle $(\Theta)$, and the $\cos$ and $\sin 3 \phi$ and $4 \phi$ residuals $\left(C_{3}, S_{3}, C_{4}, S_{4}\right)$ plotted against radii in arcseconds for our sample galaxies. The error bars for the surface brightness and colors include systematic errors from the background uncertainty. Every third error bar in the surface brightness, color, ellipticity, and position angle includes the estimated effect of the PSF. Both the $B(\square)$ and the $R(\Delta)$ data are shown for the shape and residual profiles. 

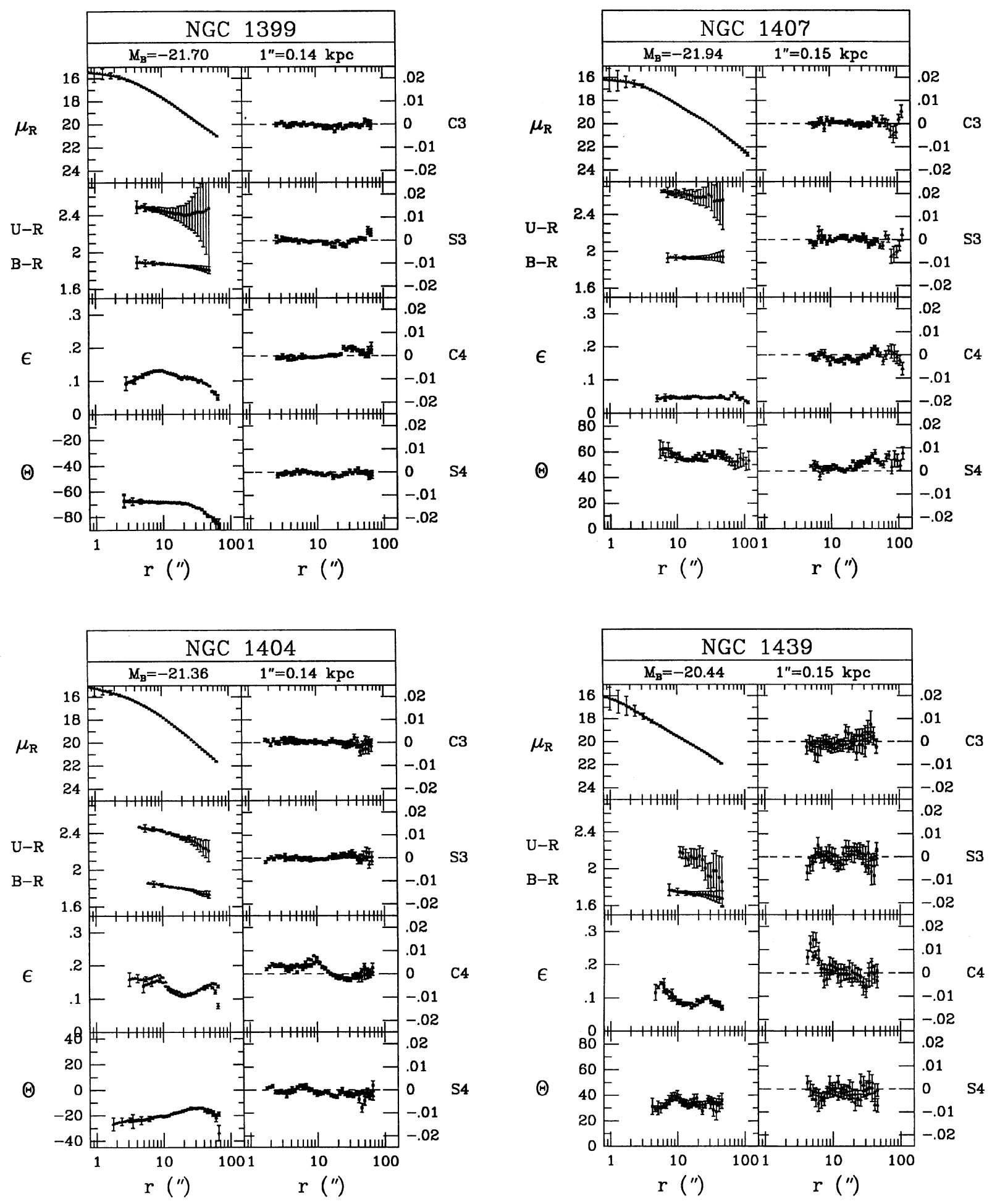

Fig. 9. (continued) 

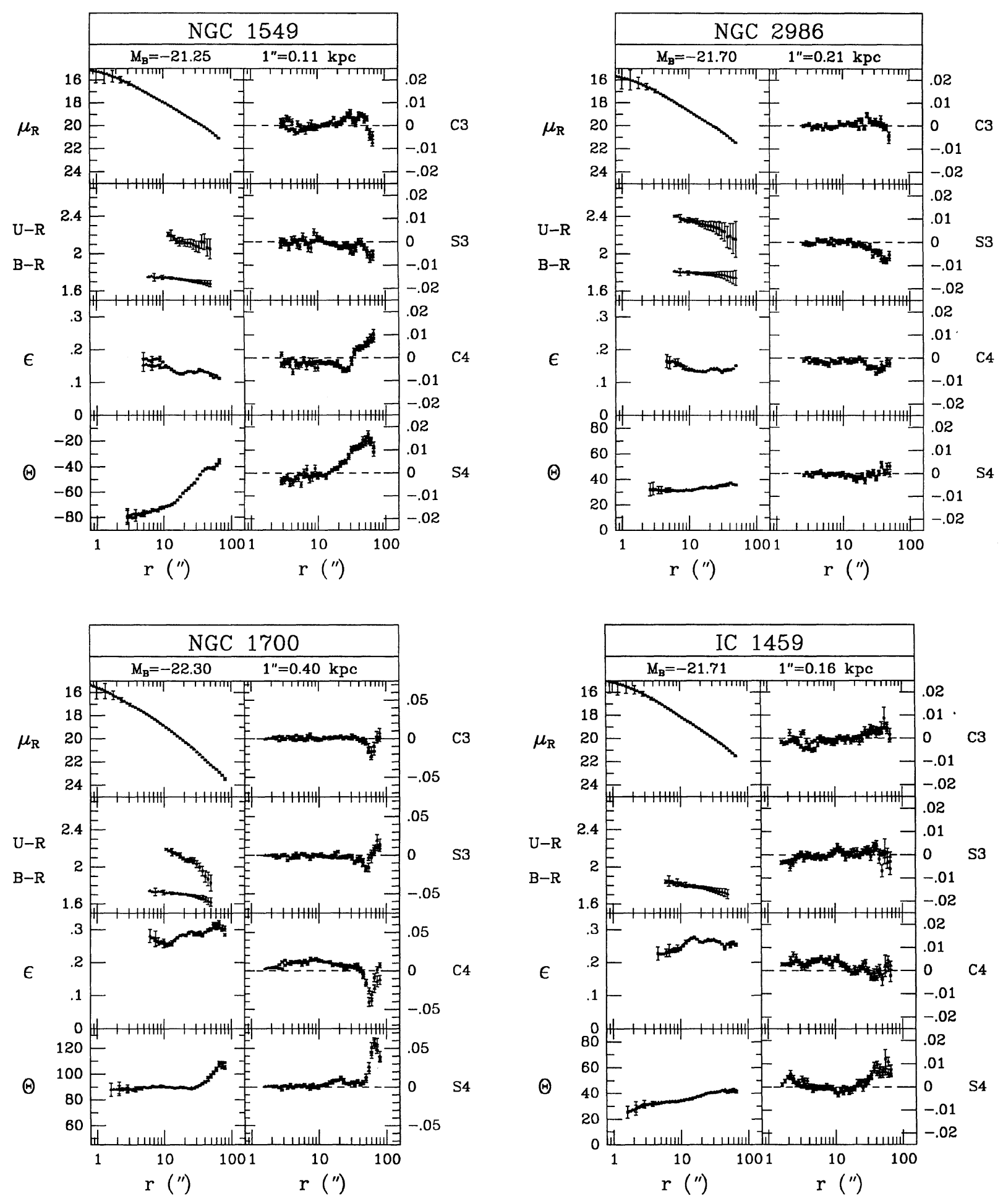

FIG. 9. (continued) 

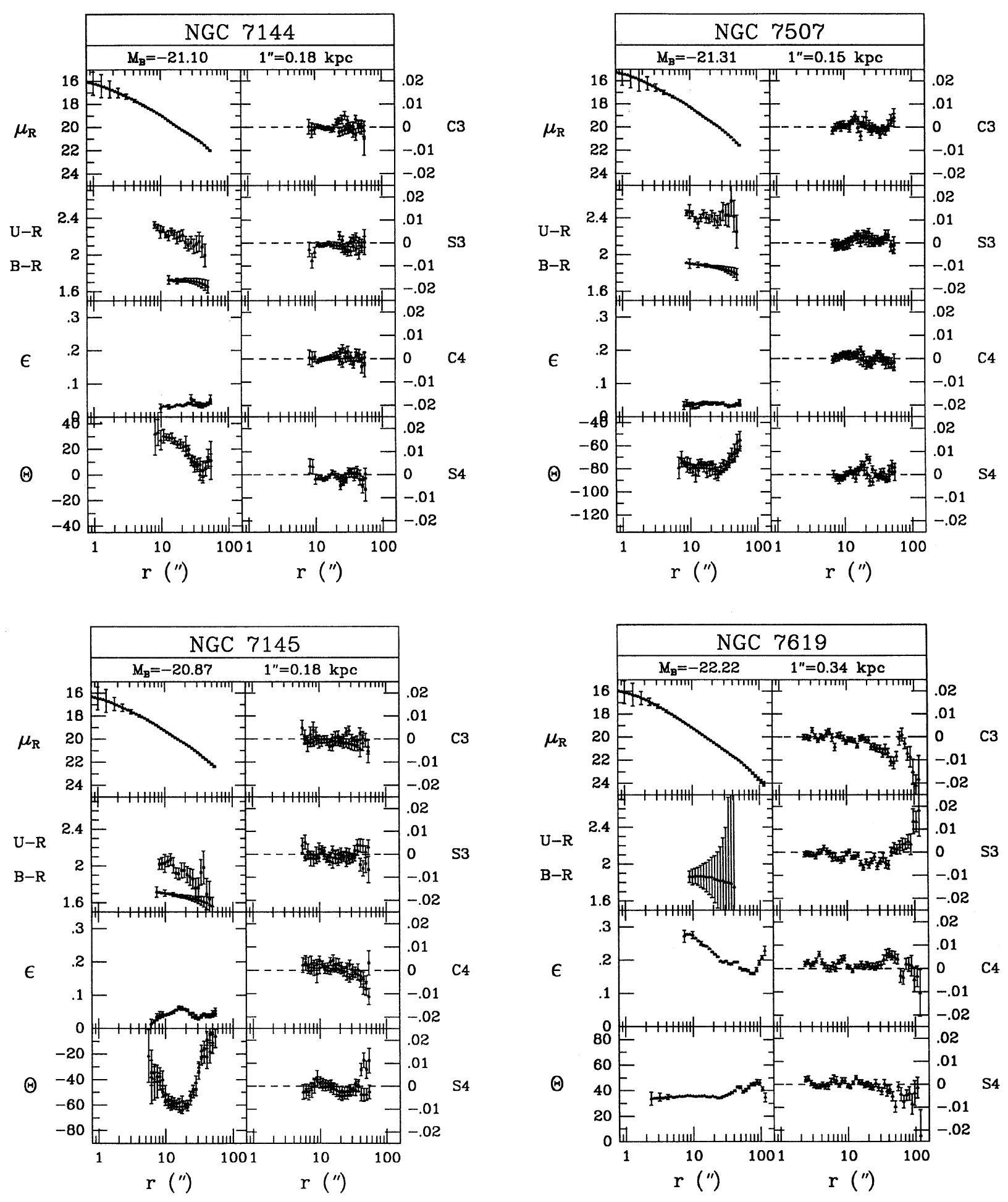

FIG. 9. (continued) 


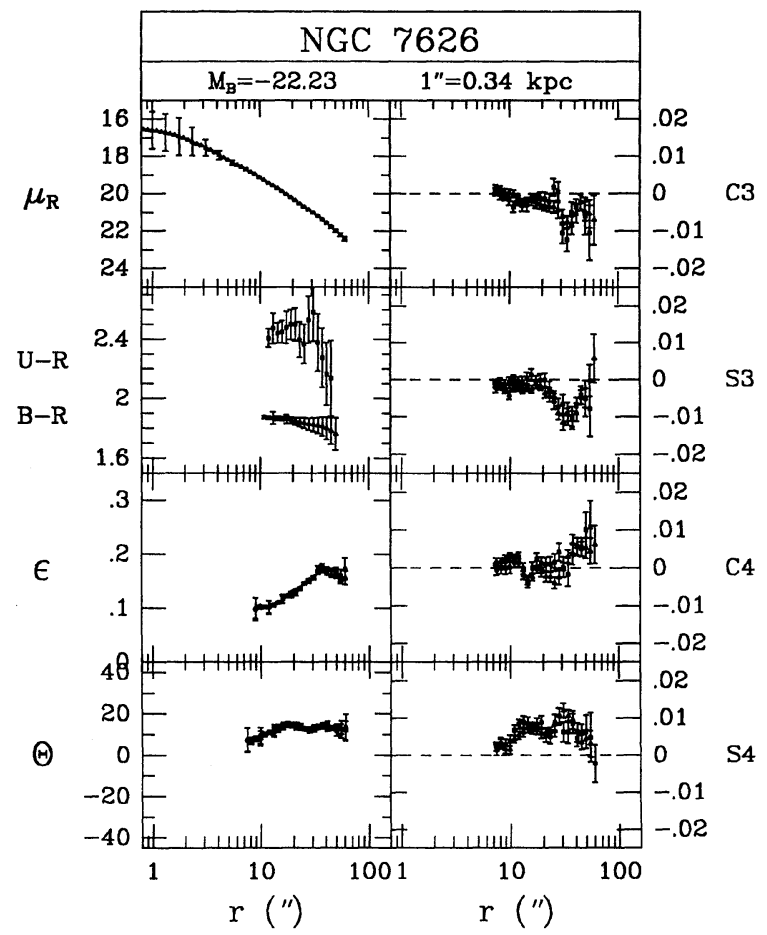

FIG. 9. (continued)

$B-R$ gradient, but we note that a constant color in $B-R$ is fully consistent with the data. Detected by $I R A S$ and at radio wavelengths.

NGC 1439. Normal elliptical from the surface photometry. Indication of a disklike distortion in the inner parts.

NGC 1549. Again, a combination of deviations from ellipses and a position-angle twist. The deviations were found before by Jedrzejewski (1987) and are discussed in the text. Malin and Carter (1983) reported faint shells. Again, the fourth-order structure has constant phase, while the major- axis position angle changes by nearly $40^{\circ}$. Detected at 100 $\mu \mathrm{m}$.

NGC 1700. The galaxy with the strongest deviations from ellipses $(5 \%)$ at a radius of $70^{\prime \prime}$, or $28 \mathrm{kpc}$. Figure 7 shows that the contours are nearly square at this radius. This galaxy is the most distant in our sample. If it were at a distance of Fornax or Virgo, the square isophotes would have fallen off the CCD! The position-angle twist and the high-order residuals are consistent with a superposition of a galaxy at constant position angle $90^{\circ}$, and a second ring or disklike component inclined at $40^{\circ}$ with respect to the galaxy.

NGC 2986. Normal elliptical, with significant, but low, residual harmonical terms, both third and fourth order. Detected at radio wavelengths.

NGC 7144. Very round elliptical. The position angle changes by $30^{\circ}$. Note that the $B$ exposure had a guiding error. We have included it because we had no other $B$ exposure, and because it shows the dramatic effects of a noncircular PSF. The magnitude of the effect was estimated quite well with our simple formulas for blurring. Marginal detection at $100 \mu \mathrm{m}$.

NGC 7145. Very round elliptical with a large positionangle twist. Malin and Carter (1983) reported shells at large radii.

IC 1459. Normal elliptical from the surface photometry, but with a counter-rotating core (e.g., Franx and Illingworth 1988). There are some deviations from ellipses at a low level $(\leqslant 0.005)$. A slightly asymmetric color image suggests the presence of dust. This galaxy has the highest IRAS $100 \mu \mathrm{m}$ flux of all, and the highest radio flux.

NGC 7507. Very round elliptical with a twist of $20^{\circ}$. A slightly asymmetric color image, indicating dust absorption. Marginal deviations from ellipses.

NGC 7619. Normal elliptical with small distortions $(\leqslant 1 \%)$. The $B$ exposure is of poor quality because of an extremely bright sky during the exposure. Radio source.

NGC 7626. Normal elliptical with distortions on a level of $1 \%$ in the third- and fourth-order harmonical residuals. This elliptical was found to have a kinematically distinct core (Jedrzejewski and Schechter 1988). Radio source.

\section{REFERENCES}

Arimoto, N., and Yoshii, Y. (1987). Astron. Astrophys. 173, 23.

Bailey, M. E., and Sparks, W. B. (1983). Mon. Not. R. Astron. Soc. 204, 53p.

Bender, R. (1988). Astron. Astrophys. Lett. 193, L7.

Bender, R., Döbereiner, S., and Möllenhof, C. (1988). Astron. Astrophys. Suppl. 74, 385.

Bender, R., and Möllenhof, C. (1987). Astron. Astrophys. 177, 71.

Binney, J. J. (1978). Comments Astrophys. 8, 27.

Binney, J. J. (1985). Mon. Not. R. Astron. Soc. 212, 767.

Binney, J. J., and Petrou, M. (1985). Mon. Not. R. Astron. Soc. 214, 449.

Boroson, T. A., and Thompson, I. B. (1987). Astron. J. 92, 33.

Boroson, T. A., Thompson, I. B., and Shectman, S. A. (1983). Astron. J. 88, 1707.

Burstein, D., Davies, R. L., Dressler, A., Faber, S. M., Stone, R. P. S., Lynden-Bell, D., Terlevich, R., and Wegner, G. (1987). Astrophys. J. Suppl. 64, 601.

Capaccioli, M., and de Vaucouleurs, G. (1983). Astrophys. J. Suppl. 52, 465.

Capaccioli, M., Piotto, G., and Rampazzo, R. (1988). Astron. J. 96, 487. Carter, D. (1987). Astrophys. J. 312, 514.

Cohen, J. G. (1986). Astron. J. 92, 1039.

Contopoulos, G. (1956). Z. Astrophys. 39, 126.
Davies, R. L. (1987). In Structure and Dynamics of Elliptical Galaxies, IAU Symposium No. 127, edited by T. de Zeeuw (Reidel, Dordrecht), p. 63.

Davies, R. L., Burstein, D., Dressler, A., Faber, S. M., Lynden-Bell, D., Terlevich, R. J., and Wegner, G. (1987). Astrophys. J. Suppl. 64, 581. Davies, R. L., and Sadler, E. M. (1987). In Structure and Dynamics of Elliptical Galaxies, IAU Symposium No. 127, edited by T. de Zeeuw (Reidel, Dordrecht), p. 441.

Davis, L. E., Cawson, M., Davies, R. L., and Illingworth, G. (1985). Astron. J. 90, 169.

de Vaucouleurs, G., and de Vaucouleurs, A. (1972). Mem. R. Astron. Soc. $77,1$.

de Vaucouleurs, G., de Vaucouleurs, A., and Corwin, H. G. (1976). Second Reference Catalogue of Bright Galaxies, Univ. Tex. Monogr. Astron. No. 2 (University of Texas, Austin) (RC2).

de Zeeuw, P. T., and Franx, M. (1989). Astrophys. J. (in press).

Disney, M. J., and Wall, J. V. (1977). Mon. Not. R. Astron. Soc. 179, 235. Djorgovski, S. (1985). Ph.D. thesis, University of California, Berkeley.

Dressel, L. L., and Condon, J. J. (1978). Astrophys. J. Suppl. 36, 53.

Ebneter, K., and Balick, B. (1985). Astron. J. 90, 183.

Ebneter, K., Djorgovski, S., and Davis, M. (1988). Astron. J. 95, 422.

Faber, S. M. (1977). Evolution of Galaxies and Stellar Populations, edited 
by B. M. Tinsley and R. B. Larson (Yale University Observatory, New Haven), p. 157.

Franx, M. (1988). Mon. Not. R. Astron. Soc. 231, 285.

Franx, M., and Illingworth, G. D. (1988). Astrophys. J. Lett. 327, L55.

Franx, M., and Illingworth, G. D. (1989). In preparation.

Franx, M., Illingworth, G. D., and Heckman, T. M. (1989). Astrophys. J. (in press)

Frogel, J. A., Persson, S. E., Aaronson, M., and Matthews, K. (1978). Astrophys. J. 220, 75.

Gorgas, J., and Efstathiou, G. (1987). In Structure and Dynamics of Elliptical Galaxies, IAU Symposium No. 127, edited by T. de Zeeuw (Reidel, Dordrecht), p. 189.

Jedrzejewski, R. I. (1987). Mon. Not. R. Astron. Soc. 226, 747.

Jedrzejewski, R. I., and Schechter, P. L. (1988). Astrophys. J. Lett. 330, L87.

Jura, M., Kim, D. W., Knapp, G. R., and Guhathakurta, P. (1987). Astrophys. J. Lett. 312, L11.

Kent, S. M. (1984). Astrophys. J. Suppl. 56, 105.

Killeen, N. E. B., and Bicknell, G. V. (1988). Astrophys. J. 325, 165.

Kondrat'ev, B. P., and Ozernoi, L. M. (1979). Sov. Astron. Lett. 5, 37.

Kormendy, J. (1984). Astrophys. J. 295, 73.

Lauberts, A., and Sadler, E. (1984). A Compilation of UBVRI Photometry for Galaxies in the ESO/Uppsala Catalogue, ESO Sci. Rep. No. 3 (ESO, Munich).

Lauer, T. R. (1985a). Astrophys. J. Suppl. 57, 473.

Lauer, T. R. (1985b). Mon. Not. R. Astron. Soc. 216, 429.
Malin, D. F., and Carter, D. (1983). Astrophys. J. 274, 534.

Mould, J. R. (1981). Publ. Astron. Soc. Pac. 93, 25.

Peletier, R. F., Illingworth, G. D., Davies, R. L., Davis, L. E., and Cawson, M. C. (1989). Preprint.

Persson, S. E., Frogel, J. A., and Aaronson, M. (1979). Astrophys. J. Suppl. 39, 61.

Sadler, E. M., and Gerhard, O. E. (1985). Mon. Not. R. Astron. Soc. 214, 177.

Sandage, A. (1973). Astrophys. J. 183, 711.

Sandage, A. (1975). Astrophys. J. 202, 563.

Sandage, A., and Tammann, G. A. (1981). A Revised Shapley Ames Catalogue of Bright Galaxies (Carnegie Institution, Washington, DC) (RSA).

Sandage, A., and Visnavathan, N. (1978). Astrophys. J. 223, 707.

Schechter, P. L. (1987). In Structure and Dynamics of Elliptical Galaxies, IAU Symposium No. 127, edited by T. de Zeeuw (Reidel, Dordrecht), p. 217.

Schweizer, F. (1979). Astrophys. J. 223, 23.

Schweizer, F. (1981). Astron. J. 86, 662.

Sparks, W. B., Wall, J. V., Thorne, D. J., Jorden, P. R., van Breda, I. G., Rudd, P. J., and Jorgensen, H. E. (1985). Mon. Not. R. Astron. Soc. 217, 87.

Statler, T. S. (1988). Astrophys. J. 331, 71.

Tonry, J. L. (1987). In Structure and Dynamics of Elliptical Galaxies, IAU Symposium No. 127, edited by T. de Zeeuw (Reidel, Dordrecht), p. 89. Whitmore, B. C., and Bell, M. (1988). Astrophys. J. 324, 741. 